\title{
Emergency care in sepsis patients
}

Citation for published version (APA):

Roest, A. A. (2016). Emergency care in sepsis patients. [Doctoral Thesis, Maastricht University]. Maastricht University. https://doi.org/10.26481/dis.20160113ar

Document status and date:

Published: 01/01/2016

DOI:

10.26481/dis.20160113ar

Document Version:

Publisher's PDF, also known as Version of record

\section{Please check the document version of this publication:}

- A submitted manuscript is the version of the article upon submission and before peer-review. There can be important differences between the submitted version and the official published version of record.

People interested in the research are advised to contact the author for the final version of the publication, or visit the DOI to the publisher's website.

- The final author version and the galley proof are versions of the publication after peer review.

- The final published version features the final layout of the paper including the volume, issue and page numbers.

Link to publication

\footnotetext{
General rights rights.

- You may freely distribute the URL identifying the publication in the public portal. please follow below link for the End User Agreement:

www.umlib.nl/taverne-license

Take down policy

If you believe that this document breaches copyright please contact us at:

repository@maastrichtuniversity.nl

providing details and we will investigate your claim.
}

Copyright and moral rights for the publications made accessible in the public portal are retained by the authors and/or other copyright owners and it is a condition of accessing publications that users recognise and abide by the legal requirements associated with these

- Users may download and print one copy of any publication from the public portal for the purpose of private study or research.

- You may not further distribute the material or use it for any profit-making activity or commercial gain

If the publication is distributed under the terms of Article $25 \mathrm{fa}$ of the Dutch Copyright Act, indicated by the "Taverne" license above, 


\section{Emergency Care in Sepsis Patients}

\section{PROEFSCHRIFT}

ter verkrijging van de graad van doctor aan de Universiteit Maastricht, op gezag van de Rector Magnificus, Prof. dr. L. L. G. Soete

volgens het besluit van het College van Decanen, in het openbaar te verdedigen

op woensdag 13 januari 2016 om 14.00 uur

door

Asselina Aaltje Roest 


\section{Promotor}

Prof. dr. C. D. A. Stehouwer

\section{Copromotor}

Dr. P. M. Stassen

\section{Beoordelingscommissie}

Prof. dr. C. P. van Schayck, voorzitter

Prof. dr. A. E. Boonen

Prof. dr. H. R. Haak

Prof. dr. H. A. H. Kaasjager, UMC Utrecht

Dr. P. W. B. Nanayakarra, VUMC Amsterdam 


\section{Contents}

Chapter 1 General introduction

Section I - The definition of sepsis

Chapter 2 The diagnostic value of the sepsis definition: good clinical judgment remains a prerequisite for diagnosing sepsis correctly

Section II - Prehospital care in sepsis patients

Chapter 3 Septic patients arriving with Emergency Medical Services:

a seriously ill population

Chapter 4

Ambulance patients with non-documented sepsis have a high mortality risk: a retrospective study

Section III - Risk stratification of sepsis patients at the Emergency Department 63

Chapter 5 Predictive accuracy and feasibility of risk stratification scores for 28-day mortality of patients with sepsis in an emergency department. 65

Chapter 6 Predictive scoring in sepsis patients at the emergency department; the abbMEDS performs best $\quad 79$

Chapter 7 Risk stratification by abbMEDS and CURB-65 in relation to treatment and disposition of the septic patient at the emergency department 101

Chapter 8 General discussion

Summary

Samenvatting

Valorisation

Appendix

Dankwoord

Curriculum Vitae 



\section{Chapter 1}

General introduction 


\section{History}

In the medical literature, the word sepsis was first mentioned by Hippocrates in the 4th century BC [1]. In ancient Greek, the word sepsis meant 'decomposition of organic matter'. Sepsis was considered the disequilibrium of the four humours (blood, phlegm, yellow bile and black bile). During the Middle Ages the theory of Galen (129-200), that sepsis and formation of pus was necessary for wound healing, prevailed [2]. Eventually, in the $19^{\text {th }}$ century, Pasteur (1822-1895) discovered micro-organisms that cause disease, also known as bacterial pathogens. Semmelweis (1818-1865) was the first to associate puerperal fever with a bacterial infection. From that moment on, the association/connection between infection and bacteria was gradually accepted. Until the $20^{\text {th }}$ century, sepsis was often referred to as 'blood poisoning' and considered to be the result of pathogen invasion of the bloodstream. In the $20^{\text {th }}$ century, the first antibiotics were discovered and developed. The first group of antibiotics were sulphonamides (1895-1964 by Domagk). Further important discoveries of antibiotics were penicillin (discovery made by Sir Alexander Fleming (18811955), Sir Howard Walter Florey (1898-1968) and Sir Ernest Boris Chain (1906-1979)) and actinomycin (Selman Waksman (1888-1973)). However, despite adequate antibiotic treatment, patients with sepsis still did not always survive. Therefore, the theory arose that not the pathogen, but the response of the host is important in the development of sepsis and the high mortality [3]. Today, sepsis is considered to be a systemic inflammatory response syndrome (SIRS) of the host to an infection [4].

\section{Definition of Sepsis}

Sepsis is a broad clinical entity and difficult to diagnose because of the complexity of its clinical presentation and the fact that there are no conclusive tests for sepsis. Therefore, in 1991, a consensus conference regarding definitions of sepsis and organ failure drafted a definition of sepsis [4]. They defined sepsis as the presence of two or more SIRS criteria and a proven or suspected infection. SIRS is defined as two or more of the following criteria; 1 ) Body temperature higher than $38.3^{\circ} \mathrm{C}$ or below $36^{\circ} \mathrm{C} ; 2$ ) Heart rate higher than 90 beats/ min; 3) Respiratory rate higher than $20 / \mathrm{min}$ or a PaCO2 lower than $4.3 \mathrm{kPa}$; 4) White blood cell count (WBC) higher than $12.10^{\circ} / \mathrm{L}$ or lower than $4.10^{9} / \mathrm{L}$ or more than $10 \%$ leukocyte bands [4]. Sepsis can be classified into three severity categories of the sepsis syndrome: sepsis, severe sepsis and septic shock [5]. Sepsis is defined as two or more SIRS criteria and a suspected or proven infection. Severe sepsis is defined as sepsis with organ dysfunction (hypotension, hypoxaemia, thrombocytopaenia, metabolic acidosis, oliguria or confusion). Septic shock is defined as severe sepsis with persisting hypotension despite adequate fluid resuscitation.

Although the current sepsis definition is highly sensitive for diagnosing sepsis, it lacks specificity [6-8]. In addition, the sepsis definition has been validated in only a few studies, which focus on intensive care patients [6, 9-12]. Therefore, most information on the value of the sepsis definition concerns a small number of patients who are more severely ill than the general population presenting at the Emergency Department $(E D)[13,14]$. 
Therefore, the discriminative ability of the sepsis definition in patients at the ED could be different from that in patients at the Intensive Care Unit (ICU) and indeed, one study found a much lower sensitivity and specificity in patients with an infection at the ED [14]. Therefore, it is important to evaluate the diagnostic value of the sepsis definition in general ED patients.

\section{Causes of Sepsis}

The vast majority of sepsis cases are caused by a bacterial infection. This can be a community-acquired or health care-associated infection. About half of all sepsis cases are caused by pneumonia, followed by intra-abdominal infections and urinary tract infections [15-17]. In only one third of the sepsis patients, blood cultures are positive and in one third of the sepsis patients, cultures from all sites (urine, sputum etc.) are negative [18]. In the ICU, the most commonly isolated micro-organism causing sepsis are gram-positive bacteria and fungi, probably because of the high number of invasive procedures [17]. In medical wards, sepsis is more frequently caused by gram-negative bacteria $[19,20]$. The most commonly isolated gram-positive bacteria are Staphylococcus aureus and Streptococcus pneumoniae. Escherichia coli, Klebsiella species and Pseudomonas aeruginosa are the most common gram-negative bacteria $[21,22]$.

\section{Epidemiology and Outcome}

Sepsis is a major health care problem, which affects millions of people worldwide [23]. The incidence of sepsis has been estimated between 15 and 19 million cases per year worldwide, and overall mortality is about $30 \%[15,23]$. The in-hospital mortality increases from $16 \%$ of the patients with uncomplicated sepsis to $46 \%$ of the patients with septic shock [24]. There are no reliable data about the overall incidence of sepsis in the Netherlands, because in 2014, the diagnosis sepsis was deleted from the Volksgezondheid Toekomst Verkenning (VTV) list of the Rijksinstituut voor Volksgezondheid en Milieu (RIVM), which means that the diagnosis sepsis is not measured and mentioned separately anymore. The only reliable data about the incidence of sepsis is derived from the National Institute of Health Care Excellence (NICE) registration. In this database, the incidence of severe sepsis at the ICU in the Netherlands is documented, which is 13,000 patients per year and about 3,500 of these patients die of severe sepsis every year [25].

Although increasing the awareness of the high prevalence and the serious nature of sepsis has received much attention, this issue is still a challenge. After the introduction of the Surviving Sepsis Campaign in 2002, which aims to improve the awareness of doctors dealing with sepsis and to provide treatment protocols, mortality decreased substantially $[26,27]$. In the Netherlands, mortality decreased with $17 \%$, while internationally, the decrease in mortality was $6 \%[26,27]$. The higher decrease in mortality in the Netherlands could in part be explained by the fact that the overall hospital mortality was higher in the Netherlands compared to the international mortality before start of the Surviving Sepsis Campaign (52 vs. $37 \%$ ). 


\section{Treatment of Sepsis}

One of the most significant recent improvements in the emergency care of sepsis patients is the introduction of early goal-directed therapy (EGDT), which is a multifaceted strategy to rapidly correct physiological derangements in sepsis patients [28]. EGDT aims to treat global tissue hypoxia by improving circulation (by administering intravenous fluids and vasoactive or inotropic agents) and oxygenation (by administering oxygen and in case of a low haemoglobin concentration, a red blood cell transfusion). Implementation of EGDT has reduced mortality and sepsis-related costs $[26,28,29]$. EGDT was already used in cardiac surgery and in sepsis patients at the ICU, but in 2001, Rivers et al. showed that it also was effective when implemented in patients with severe sepsis and septic shock at the Emergency Department (ED) [28]. This makes sense if you look at the sequence SIRS, sepsis, severe sepsis and septic shock as a process of falling dominoes.

From this moment on, international initiatives resulted in the Surviving Sepsis Campaign in 2002 which had the goal to reduce mortality from sepsis by $25 \%$ in 5 years [30]. Cornerstones of this program, among others, were increasing the use of appropriate treatment and providing guidelines of care, one of this was EGDT. EGDT consists of aggressive fluid resuscitation, supplemental oxygen, blood transfusion and use of vasoactive agents [28]. However, two recent controlled studies have shown no benefit of EGDT [31, 32]. This may be explained by the fact that EGDT probably has become part of the current usual care in sepsis patients, thereby reducing the differences in outcome between the treatment and usual care group. This seems indeed likely, because in these two studies, the observed mortality in both the treatment and control group was lower than expected (based on the calculation of their severity of illness). However, EGDT is in fact resuscitation of the patient and correction of abnormal vital signs, and hence leads to disappearance of SIRS (abnormal heart rate, abnormal respiratory rate) and not of the underlying infection. Therefore, it is also important to treat the underlying infection by administering antibiotics immediately after identifying a patient with sepsis. Indeed, in an uncontrolled study, every hour of delay in the administration of antibiotics in patients with septic shock was associated with an increase of mortality of 7.6\% [33]. In order to avoid an increase in mortality caused by bacterial resistance, broad spectrum antibiotics are used. Therefore, the Surviving Sepsis Campaign advices administration of broad spectrum antibiotics as soon as possible, but at least within 3 hours of presentation at the ED [24].

\section{Prehospital Care in Sepsis}

The guidelines for treatment provided by the Surviving Sepsis Campaign only address the treatment of the septic patients after arrival at the hospital and not during the pre-hospital phase [34]. This focus on the hospital phase leaves room to improve the recognition and initiation of treatment of the sepsis patient in the pre-hospital phase. There are only a few studies concerning the care of sepsis patients by ambulance personnel [35]. In the United States of America, patients with sepsis who were transported by ambulance were more seriously ill, and had a higher chance of dying than patients who arrived at the ED otherwise [36-38]. Early treatment in the ambulance decreased mortality and the number 
of intubations after arrival at the ED [39]. Interestingly, these ambulance patients were not only treated earlier by ambulance personnel, but also during stay at the ED [37]. Thus, transportation by ambulance not only offers an opportunity to treat sepsis patients early in the ambulance, but also accelerates the treatment after arrival at the hospital. These advantages for sepsis patients can, however, only be achieved when these patients are transported by ambulance and when sepsis is recognized in the ambulance. Unfortunately, severe sepsis was recognized in only $48 \%$ of the cases by ambulance staff, even after a training that aimed to improve recognition of sepsis [40]. Therefore, it is important to establish why sepsis is not recognized by ambulance staff and which factors influence recognition.

\section{Risk Stratification at the ED}

As mentioned before, treatment in sepsis patients is most effective when started early [33]. This finding underlines the importance of identifying sepsis patients with the highest chance of dying in an early stage. However, severity of disease is often underestimated in sepsis patients [41, 42]. Therefore, an easy and accurate scoring system that quantifies the severity of illness and quickly predicts a patient's risk of dying may be helpful to make decisions about treatment by increasing the treating physician's awareness of the risks that threaten the patient. Several scores that quantify the severity of illness in sepsis patients have been developed and validated in different populations and countries (reviewed by Challen, et al. and Calle, et al.) $[43,44]$. However, these scores are often designed and validated in the same cohort, which may lead to an overestimation of their discriminatory value [45-47]. In addition, the scores are often not calibrated $[45,48]$. Further, most scores are developed in ICU patients with severe sepsis and are therefore not automatically useful in the emergency care of sepsis patients at the $\operatorname{ED}[49,50]$.

Implementation of severity scores in sepsis patients at the ED is only possible after evaluation of the several existing scores in a large population of sepsis patients at the ED. First, it is important to evaluate the discriminatory value and feasibility of the current scores. Second, we have to determine which score is calibrated best in sepsis patients at the ED.

Further, although some of the scores include items regarding age, the predictive value of the scores has not been established in the group of elderly patients, who form a major part of the patients with sepsis. Due to ageing of the population and their high comorbidity, there is an increase of elderly patients visiting the ED. One study concerning the very elderly with sepsis shows that the usual predictors of in-hospital mortality (age, higher number of co-morbidities and poor mobility) do not apply to them [51]. Therefore, it is important to evaluate the accuracy of the existing severity of illness scores in sepsis patients in different age categories.

Besides predictive scores, in daily practice, traditional inflammation markers such as C-reactive protein (CRP) and white blood cell count (WBC) are used to assess the severity of disease. Two studies reported that CRP was higher in survivors than in non-survivors in elderly patients $[52,53]$. The association between WBC and mortality has not yet been established $[54,55]$. Because of their use in daily practice it is important to establish whether or not these inflammation markers can predict mortality in sepsis patients at the ED. 
Furthermore, a sepsis-specific ED score would ideally be part of a clinical decision support system that guides antibiotic treatment and disposition. Eventually, this may lead to a higher level of care in sepsis patients. For patients with community-acquired pneumonia (CAP), such a score already exists. The CURB-65 score (Confusion, Urea, Respiration, Blood pressure, Age $>65$ years) not only stratifies patients into three risk categories and predicts 28-day mortality rather accurately [56], but also provides clinical guidance for the physician. For each risk category, it advises on two management decisions, namely choice of antibiotics (e.g. small or broad spectrum) and disposition (i.e. whether a patient should be admitted to the hospital and if so to what type of ward) $[57,58]$. For patients with sepsis, no such score exists. It would be ideal, if one of the sepsis scores, like the abbMEDS [59], derived from the MEDS score [39], which is a good and feasible score, could provide the same clinical guidance for sepsis as the CURB-65 does for CAP. Since there is an overlap between sepsis and CAP, we hypothesize that both the abbMEDS and CURB-65 risk categories can be related to decisions concerning management in sepsis patients. However, this has never been investigated before.

\section{Aims and Outline of this Thesis}

Sepsis affects millions of people worldwide and has a high mortality rate, which may be influenced in a positive way by early start of treatment. However, before the start of treatment, sepsis has to be recognized/diagnosed and the severity of sepsis has to be evaluated as well. Therefore, this thesis aims to investigate the diagnostic accuracy of the definition of sepsis, the recognition of sepsis patients in the prehospital phase and the use of risk stratification scores in sepsis patients at the ED.

First, in chapter 2, the diagnostic value of the sepsis definition in a general internal medicine population at the Emergency Department is described. Furthermore, a subgroup analysis in patients with a suspected or proven infection is performed. In addition, the diagnosis sepsis during hospital stay is evaluated.

Second, as treatment is most effective when started early, Chapter 3 evaluates whether the ambulance can play a role in the early start of this treatment. Therefore, as a first step, an analysis is performed on how many and which sepsis patients are transported by ambulance and whether or not they were documented as having sepsis. In chapter 4, documentation of sepsis by ambulance personnel is investigated. The factors that influence documentation and mortality are evaluated.

Third, sepsis is a broad clinical entity with a wide range in mortality. So, after recognition, it is important to assess the severity of disease and mortality risk. In chapter $\mathbf{5}$, the discriminatory value of five risk stratification scores in predicting 28-day mortality are assessed. In the last couple of years, a large number of new risk stratification scores have been developed and validated. In addition, in daily practice, white blood cell count (WBC) and C-reactive protein (CRP) are often used to assess severity of sepsis. Therefore, in chapter 6 , a comparative analysis is made of 14 risk stratification scores and the two laboratory values (WBC and CRP). Little is known about the performance of the risk stratification scores in elderly patients. So, in order to assess whether the discriminatory value of the best scores are age-dependent, the value of these scores are compared at different cut-off 
points ( $<65, \geq 65, \geq 75$ and $\geq 85$ years of age). It would be ideal if the risk stratification scores would also provide guidance regarding clinical decisions, such as antibiotic treatment and disposition. Therefore, in chapter 7, a preliminary analysis regarding the ability of the abbMEDS and CURB-65 to provide clinical decision support is described.

Finally, in chapter $\mathbf{8}$, the main findings of this thesis, as well as its limitations, the relevance and implications for current clinical practice are discussed. 


\section{References}

1. Majno G. The ancient riddle of sigma eta psi iota sigma (sepsis). J Infect Dis. 1991;163(5):937-45.

2. Funk DJ, Parrillo JE, Kumar A. Sepsis and septic shock: a history. Crit Care Clin. 2009;25(1):83-101, viii.

3. Cerra FB. The systemic septic response: multiple systems organ failure. Crit Care Clin. 1985;1(3):591607.

4. Bone RC, Sibbald WJ, Sprung CL. The ACCP-SCCM consensus conference on sepsis and organ failure. Chest. 1992;101(6):1481-3.

5. Levy MM, Fink MP, Marshall JC, Abraham E, Angus D, Cook D, et al. 2001 SCCM/ESICM/ACCP/ATS/SIS International Sepsis Definitions Conference. Crit Care Med. 2003;31(4):1250-6.

6. Vincent JL, Opal SM, Marshall JC, Tracey KJ. Sepsis definitions: time for change. Lancet. 2013;381(9868):774-5.

7. Balk RA. Systemic inflammatory response syndrome (SIRS): where did it come from and is it still relevant today? Virulence. 2014;5(1):20-6.

8. Klein Klouwenberg PM, Ong DS, Bonten MJ, Cremer OL. Classification of sepsis, severe sepsis and septic shock: the impact of minor variations in data capture and definition of SIRS criteria. Intensive Care Med. 2012;38(5):811-9.

9. Alberti C, Brun-Buisson C, Burchardi H, Martin C, Goodman S, Artigas A, et al. Epidemiology of sepsis and infection in ICU patients from an international multicentre cohort study. Intensive Care Med. 2002;28(2):108-21.

10. Brun-Buisson C, Meshaka P, Pinton P, Vallet B, Group ES. EPISEPSIS: a reappraisal of the epidemiology and outcome of severe sepsis in French intensive care units. Intensive Care Med. 2004;30(4):580-8.

11. Kaukonen KM, Bailey M, Pilcher D, Cooper DJ, Bellomo R. Systemic inflammatory response syndrome criteria in defining severe sepsis. N Engl J Med. 2015;372(17):1629-38.

12. Sprung CL, Sakr Y, Vincent JL, Le Gall JR, Reinhart K, Ranieri VM, et al. An evaluation of systemic inflammatory response syndrome signs in the Sepsis Occurrence In Acutely III Patients (SOAP) study. Intensive Care Med. 2006;32(3):421-7.

13. Jaimes F, Garces J, Cuervo J, Ramirez F, Ramirez J, Vargas A, et al. The systemic inflammatory response syndrome (SIRS) to identify infected patients in the emergency room. Intensive Care Med. 2003;29(8):1368-71.

14. Gille-Johnson P, Hansson KE, Gardlund B. Severe sepsis and systemic inflammatory response syndrome in emergency department patients with suspected severe infection. Scand J Infect Dis. 2013;45(3):186-93.

15. Angus DC, Linde-Zwirble WT, Lidicker J, Clermont G, Carcillo J, Pinsky MR. Epidemiology of severe sepsis in the United States: analysis of incidence, outcome, and associated costs of care. Crit Care Med. 2001;29(7):1303-10.

16. Vincent JL, Rello J, Marshall J, Silva E, Anzueto A, Martin CD, et al. International study of the prevalence and outcomes of infection in intensive care units. JAMA. 2009;302(21):2323-9.

17. Angus DC, van der Poll T. Severe sepsis and septic shock. N Engl J Med. 2013;369(21):2063.

18. Hoeboer SH, van der Geest PJ, Nieboer D, Groeneveld AB. The diagnostic accuracy of procalcitonin for bacteraemia: a systematic review and meta-analysis. Clin Microbiol Infect. 2015;21(5):474-81.

19. Mearelli F, Fiotti N, Altamura N, Zanetti M, Fernandes G, Burekovic I, et al. Heterogeneous models for an early discrimination between sepsis and non-infective SIRS in medical ward patients: a pilot study. Intern Emerg Med. 2014;9(7):749-57.

20. Rohde JM, Odden AJ, Bonham C, Kuhn L, Malani PN, Chen LM, et al. The epidemiology of acute organ system dysfunction from severe sepsis outside of the intensive care unit. J Hosp Med. 2013;8(5):2437.

21. Opal SM, Garber GE, LaRosa SP, Maki DG, Freebairn RC, Kinasewitz GT, et al. Systemic host responses in severe sepsis analyzed by causative microorganism and treatment effects of drotrecogin alfa (activated). Clin Infect Dis. 2003;37(1):50-8.

22. Ranieri VM, Thompson BT, Barie PS, Dhainaut JF, Douglas IS, Finfer S, et al. Drotrecogin alfa (activated) in adults with septic shock. N Engl J Med. 2012;366(22):2055-64.

23. Adhikari NK, Fowler RA, Bhagwanjee S, Rubenfeld GD. Critical care and the global burden of critical 
illness in adults. Lancet. 2010;376(9749):1339-46.

24. Rangel-Frausto MS, Pittet D, Costigan M, Hwang T, Davis CS, Wenzel RP. The natural history of the systemic inflammatory response syndrome (SIRS). A prospective study. JAMA. 1995;273(2):117-23.

25. Anonymous. www.vmszorg.nl/themas/sepsis.

26. Levy MM, Dellinger RP, Townsend SR, Linde-Zwirble WT, Marshall JC, Bion J, et al. The Surviving Sepsis Campaign: results of an international guideline-based performance improvement program targeting severe sepsis. Crit Care Med. 2010;38(2):367-74.

27. Tromp M, Tjan DH, van Zanten AR, Gielen-Wijffels SE, Goekoop GJ, van den Boogaard M, et al. The effects of implementation of the Surviving Sepsis Campaign in the Netherlands. Neth J Med. 2011;69(6):292-8.

28. Rivers E, Nguyen B, Havstad S, Ressler J, Muzzin A, Knoblich B, et al. Early goal-directed therapy in the treatment of severe sepsis and septic shock. N Engl J Med. 2001;345(19):1368-77.

29. Puskarich MA, Marchick MR, Kline JA, Steuerwald MT, Jones AE. One year mortality of patients treated with an emergency department based early goal directed therapy protocol for severe sepsis and septic shock: a before and after study. Crit Care. 2009;13(5):R167.

30. Anonymous. http://www.survivingsepsis.org/About-SSC/Pages/History.aspx.

31. Pro $\mathrm{Cl}$, Yealy DM, Kellum JA, Huang DT, Barnato AE, Weissfeld LA, et al. A randomized trial of protocolbased care for early septic shock. N Engl J Med. 2014;370(18):1683-93.

32. Investigators A, Group ACT, Peake SL, Delaney A, Bailey M, Bellomo R, et al. Goal-directed resuscitation for patients with early septic shock. N Engl J Med. 2014;371(16):1496-506.

33. Kumar A, Ellis P, Arabi Y, Roberts D, Light B, Parrillo JE, et al. Initiation of inappropriate antimicrobial therapy results in a fivefold reduction of survival in human septic shock. Chest. 2009;136(5):1237-48.

34. Dellinger RP, Levy MM, Rhodes A, Annane D, Gerlach H, Opal SM, et al. Surviving Sepsis Campaign: international guidelines for management of severe sepsis and septic shock, 2012. Intensive Care Med. 2013;39(2):165-228.

35. van der Wekken LC, Alam N, Holleman F, van Exter P, Kramer MH, Nanayakkara PW. Epidemiology of Sepsis and Its Recognition by Emergency Medical Services Personnel in the Netherlands. Prehosp Emerg Care. 2015.

36. Band RA, Gaieski DF, Hylton JH, Shofer FS, Goyal M, Meisel ZF. Arriving by emergency medical services improves time to treatment endpoints for patients with severe sepsis or septic shock. Acad Emerg Med. 2011;18(9):934-40.

37. Studnek JR, Artho MR, Garner CL, Jr., Jones AE. The impact of emergency medical services on the ED care of severe sepsis. Am J Emerg Med. 2012;30(1):51-6.

38. Seymour CW, Band RA, Cooke CR, Mikkelsen ME, Hylton J, Rea TD, et al. Out-of-hospital characteristics and care of patients with severe sepsis: a cohort study. J Crit Care. 2010;25(4):553-62.

39. Seymour CW, Cooke CR, Mikkelsen ME, Hylton J, Rea TD, Goss CH, et al. Out-of-hospital fluid in severe sepsis: effect on early resuscitation in the emergency department. Prehosp Emerg Care. 2010;14(2):145-52.

40. Guerra WF, Mayfield TR, Meyers MS, Clouatre AE, Riccio JC. Early detection and treatment of patients with severe sepsis by prehospital personnel. J Emerg Med. 2013;44(6):1116-25.

41. Assuncao M, Akamine N, Cardoso GS, Mello PV, Teles JM, Nunes AL, et al. Survey on physicians' knowledge of sepsis: do they recognize it promptly? J Crit Care. 2010;25(4):545-52.

42. Cronshaw HL, Daniels R, Bleetman A, Joynes E, Sheils M. Impact of the Surviving Sepsis Campaign on the recognition and management of severe sepsis in the emergency department: are we failing? Emerg Med J. 2011;28(8):670-5.

43. Challen K, Goodacre SW. Predictive scoring in non-trauma emergency patients: a scoping review. Emerg Med J. 2011;28(10):827-37.

44. Calle P, Cerro L, Valencia J, Jaimes F. Usefulness of severity scores in patients with suspected infection in the emergency department: a systematic review. J Emerg Med. 2012;42(4):379-91.

45. Howell MD, Donnino MW, Talmor D, Clardy P, Ngo L, Shapiro NI. Performance of severity of illness scoring systems in emergency department patients with infection. Acad Emerg Med. 2007;14(8):709-14.

46. Olsson T, Lind L. Comparison of the rapid emergency medicine score and APACHE II in nonsurgical emergency department patients. Acad Emerg Med. 2003;10(10):1040-8.

47. Shapiro NI, Wolfe RE, Moore RB, Smith E, Burdick E, Bates DW. Mortality in Emergency Department 
Sepsis (MEDS) score: a prospectively derived and validated clinical prediction rule. Crit Care Med. 2003;31(3):670-5.

48. Chen YX, Li CS. Risk stratification and prognostic performance of the predisposition, infection, response, and organ dysfunction (PIRO) scoring system in septic patients in the emergency department: a cohort study. Crit Care. 2014;18(2):R74.

49. Knaus WA, Draper EA, Wagner DP, Zimmerman JE. APACHE II: a severity of disease classification system. Crit Care Med. 1985;13(10):818-29.

50. Vincent JL, Moreno R, Takala J, Willatts S, De Mendonca A, Bruining H, et al. The SOFA (Sepsis-related Organ Failure Assessment) score to describe organ dysfunction/failure. On behalf of the Working Group on Sepsis-Related Problems of the European Society of Intensive Care Medicine. Intensive Care Med. 1996;22(7):707-10.

51. Pai Y, Butchart C, Lunt CJ, Musonda P, Gautham N, Soiza RL, et al. Age, co-morbidity and poor mobility: no evidence of predicting in-patient death and acute hospital length of stay in the oldest old. QJM. 2011;104(8):671-9.

52. Povoa P. C-reactive protein: a valuable marker of sepsis. Intensive Care Med. 2002;28(3):235-43.

53. Cox ML, Rudd AG, Gallimore R, Hodkinson HM, Pepys MB. Real-time measurement of serum C-reactive protein in the management of infection in the elderly. Age Ageing. 1986;15(5):257-66.

54. Suberviola B, Castellanos-Ortega A, Gonzalez-Castro A, Garcia-Astudillo LA, Fernandez-Miret B. [Prognostic value of procalcitonin, C-reactive protein and leukocytes in septic shock]. Med Intensiva. 2012;36(3):177-84

55. Magrini L, Gagliano G, Travaglino F, Vetrone F, Marino R, Cardelli P, et al. Comparison between white blood cell count, procalcitonin and $C$ reactive protein as diagnostic and prognostic biomarkers of infection or sepsis in patients presenting to emergency department. Clin Chem Lab Med. 2014;52(10):1465-72.

56. Lim WS, van der Eerden MM, Laing R, Boersma WG, Karalus N, Town GI, et al. Defining community acquired pneumonia severity on presentation to hospital: an international derivation and validation study. Thorax. 2003;58(5):377-82.

57. Harris M, Clark J, Coote N, Fletcher P, Harnden A, McKean M, et al. British Thoracic Society guidelines for the management of community acquired pneumonia in children: update 2011. Thorax. 2011;66 Suppl 2:ii1-23.

58. Wiersinga WJ, Bonten MJ, Boersma WG, Jonkers RE, Aleva RM, Kullberg BJ, et al. SWAB/NVALT (Dutch Working Party on Antibiotic Policy and Dutch Association of Chest Physicians) guidelines on the management of community-acquired pneumonia in adults. Neth J Med. 2012;70(2):90-101.

59. Vorwerk C, Loryman B, Coats TJ, Stephenson JA, Gray LD, Reddy G, et al. Prediction of mortality in adult emergency department patients with sepsis. Emerg Med J. 2009;26(4):254-8. 


\section{Section I}

The definition of sepsis 



\section{Chapter 2}

The diagnostic value of the sepsis definition: good clinical judgment remains a prerequisite for diagnosing sepsis correctly

Asselina A. Roest, Joris J. Heyligen; Jan Tegtmeier, Hella F. Borggreve; Nikki Troost, Marloes G.B. van Onna, Andrea Peeters, Coen D.A. Stehouwer, Patricia M. Stassen

Submitted 


\begin{abstract}
Objective

The sepsis definition is consensus based, but still subject to discussion. We aimed to assess the diagnostic value of the sepsis definition in an Emergency Department (ED) population.

\section{Methods}

We retrospectively compared the sepsis definition and face diagnosis (diagnosis at the ED) with the discharge diagnosis (reference test) regarding diagnostic value in patients admitted by the internist. We further retrieved how many patients received the correct diagnosis over time yielded by both sepsis definition and face diagnosis. Last, factors explaining differences between the sepsis definition and discharge diagnosis were analysed.
\end{abstract}

\title{
Results
}

In total, 538 patients were included. Of the 181 (33.6\%) patients with $\geq 2$ SIRS criteria, 139 (76.8\%) had an infection. Compared to the discharge diagnosis, the sepsis definition had lower diagnostic value than the face diagnosis (sensitivity 73.5 vs. $81.4 \%$, specificity 86.8 vs. $95.0 \%$, AUC 0.80 (95\%Cl0.75-0.85) vs. 0.88 ( $95 \% \mathrm{Cl} 0.84-0.93)$, respectively, $\mathrm{p}<0.001$ ). In total, 75/139 (54.0\%) patients who fulfilled the sepsis definition at the ED, received both the face and discharge diagnosis sepsis. In addition, the sepsis definition diagnosed $56 / 139(40.3 \%)$ patients wrongly with sepsis and 30/399 (7.5\%) were missed by the sepsis definition. This discordance in diagnosis could not be explained by differences in age, comorbidity, vital signs or laboratory values.

\section{Conclusion}

The sepsis definition had less diagnostic value than the face diagnosis when the discharge diagnosis was used as reference. Good clinical judgment remains a prerequisite for diagnosing sepsis correctly. 


\section{Introduction}

Despite significant advances in understanding and management, sepsis remains a major cause of morbidity and mortality, especially when accompanied by organ failure $[1,2]$. Early recognition of sepsis facilitates adequate treatment at an early stage [3], which ultimately reduces mortality in patients with severe sepsis and septic shock $[4,5]$. In order to intervene at an early stage, highly sensitive diagnostic criteria and/or tests are needed to recognize sepsis patients in time.

Therefore, a definition of sepsis was established in 1991 by Bone, et al. [6]. Sepsis was defined as a Systemic Inflammatory Response Syndrome (SIRS) in response to an infection. The SIRS criteria consist of four items: body temperature, respiratory rate or $\mathrm{pCO} 2$, heart rate and white blood cell count or leucocyte bands. The sepsis definition consists of two or more SIRS criteria that occur in response to an infection [6]. Although this definition has been implemented worldwide, there are growing concerns about the lack of specificity in clinical practice [7-9], for even a sterile inflammatory response caused by, for instance, a pancreatitis, can mimic the signs of SIRS [10]. Low specificity could lead to overtreatment, and thereby to bacterial resistance, and to missing of other important diagnoses, such as a pulmonary embolism. Despite this drawback of the sepsis definition, it was concluded at the International Sepsis Definitions Conference of 2001 that there was not enough evidence to change the way sepsis was defined $[8,13]$.

The sepsis definition has been validated in only a few studies with a focus on intensive care patients $[11,12]$. Therefore, most information concerns patients who are more severely ill than the general population presenting at the Emergency Department (ED) [13, 14].

In this retrospective study, we first aimed to assess the prevalence of patients fulfilling the SIRS criteria upon arrival at the ED in a general internal medicine population. Second, we aimed to compare the diagnostic value of the sepsis definition upon arrival at the ED with the diagnosis of the physician at the ED and the diagnosis at discharge from the hospital. Third, we aimed to assess the diagnostic value of the SIRS criteria in a subgroup of patients with a suspected or proven infection, for the clinician is probably most interested in the diagnostic value of these SIRS criteria in the context of a suspected/proven infection. Fourth, we aimed to investigate changes in the diagnosis sepsis over time, i.e. the number patients with changed diagnoses during their stay at the ED and the hospital. We investigated this because we expected that changes in the diagnosis occur over time, because of evolving symptoms of disease and/or increasing availability of test results. Last, we aimed to investigate the reason for inconsistencies (if present) between the sepsis definition and discharge diagnosis. 


\section{Materials and methods}

\section{Design and setting}

This retrospective study was performed at the ED of the Maastricht University Medical Centre + $(\mathrm{MUMC}+)$. The MUMC+ is a secondary and tertiary care medical centre in the Netherlands. The ED is visited by approximately 23,000 patients every year, of which around 5500 patients are assessed by internists and their residents. In our hospital, internists specialized in acute care assess all medical patients who present to the emergency department (ED). These include patients with general internal medicine, endocrine, oncological, haematological, nephrological, gastrointestinal or rheumatological medical issues. General practitioners (whose service is available 24/7) refer the majority of patients, while some patients (high urgency patients) arrive by ambulance and a minority of patients are self-presenters (for more details on the organization of acute care in the Netherlands, see Thijssen, et al.)[15]. The study was approved by the MUMC+ Medical Ethics Committee.

\section{Patients}

We included all patients $>17$ years of age who were admitted by the internist to the hospital via the ED in the last week of every month from January 2011 until December 2011. All patients who were referred to the internist at the ED but who were admitted to another department than internal medicine were excluded. Only the first ED visit of patients was included in the analysis.

\section{Data collection}

All data were retrieved from the patients' ED and hospital charts using a standardized extraction form. The SIRS criteria, information on the presence of proven/suspected infection, the diagnosis at the ED made by the physician (face diagnosis) and the final diagnosis at the moment of discharge (discharge diagnosis) were retrieved. To determine whether the diagnosis of sepsis was made, either at the ED or at discharge, two investigators evaluated the patients' charts independently. In case of disagreement, the judgment of a third investigator was decisive. For all patients, information regarding demographic data, vital parameters, laboratory, microbiology and diagnostic imaging results, fulfilment of the SIRS criteria upon arrival at the ED and items of the Charlson comorbidity index $(\mathrm{CCl})$ ) was retrieved [16]. Only the microbiology and radiologic examination results collected during the three first days after admission were included, to ensure that hospital-acquired infections were excluded. Information regarding mortality rates during hospital stay, within 28 days and within one year after admission was also included. Mortality data from the municipal administration are linked to the electronic patient chart.

Because our data collection relied on retrospective assessment of patients' charts, not all values for the SIRS criteria were complete. When respiratory rate and pCO2-levels were missing, we estimated the respiratory rate using physician reports on respiratory rate, in 
accordance with other studies [17]. When no indication of abnormal values was reported, the SIRS criteria were considered normal.

\section{Definitions}

The SIRS criteria measured at the ED are defined as: 1) Body temperature higher than $38.3^{\circ} \mathrm{C}$ or below $36^{\circ} \mathrm{C} ; 2$ ) Heart rate higher than 90 beats/min; 3) Respiratory rate higher than $20 / \mathrm{min}$ or a PaCO2 lower than $4.3 \mathrm{kPa}$; 4) White blood cell count (WBC) higher than $12.10^{9} / \mathrm{L}$ or lower than $4.10^{9} / \mathrm{L}$ or more than $10 \%$ leukocyte bands $[8,13]$.

Suspected or proven infection: the documentation of a suspected or proven infection in the ED chart.

Sepsis definition: the presence of $\geq 2$ SIRS criteria and a proven or suspected infection at the ED [6].

Face diagnosis: diagnosis made by using pattern recognition by the physician at the ED.

Discharge diagnosis: discharge diagnosis recorded in the patients' charts. When a patient developed sepsis after stay in the hospital for more than 24 hours, we considered the patient as not septic in order to excluded hospital acquired sepsis, which was not present at the ED. We considered this diagnosis the "reference test" for the diagnosis of sepsis, because at discharge, most results of laboratory, cultures and radiologic examinations are known.

\section{Analysis}

To assess the diagnostic value of the sepsis definition, we conducted four analyses upon arrival at the ED. First, all patients were divided into two groups; one group with $\geq 2$ SIRS criteria and one with $<2$ SIRS criteria. We evaluated whether these two groups differed regarding the prevalence of infection, the number of comorbid conditions and mortality rate (in-hospital, 28-day and 1-year).

Second, we compared the sepsis definition, the face diagnosis and a combination of the two tests 'sepsis by sepsis definition or face diagnosis' with the discharge diagnosis (reference test). In addition to this, we divided the sepsis definition into two different tests, ' $\geq 2$ SIRS criteria' and 'presence of suspected or proven infection', which we both compared with the discharge diagnosis. To assess the diagnostic value of the aforementioned tests, we calculated sensitivity, specificity, positive predictive value (PPV), negative predictive value (NPV) and the corresponding area under the Receiver Operating Characteristic-curve (AUC) with $95 \%$ Confidence Interval $(95 \% \mathrm{Cl})$.The AUCs of the tests were compared with each other.

Third, we conducted a subgroup analysis in the patients with a suspected or proven infection at the ED. In daily clinical practice, physicians are probably most interested in the SIRS criteria when they suspect the patient to have an infection. In this subgroup, we investigated the diagnostic value of the number of present SIRS criteria $(\geq 1, \geq 2, \geq 3$ and 4$)$ and of the number of present SIRS criteria consisting of vital signs only $(\geq 1, \geq 2$ and 3$)$.

Fourth, to gain insight into the diagnosis of sepsis over time, we constructed a flowchart in which we showed how many patients crossed-over from the diagnosis sepsis to 'no sepsis' according to the sepsis definition, the face diagnosis and the discharge diagnosis. 
Last, in the group of patients who fulfilled the sepsis definition, but not the discharge diagnosis or vice-versa, we investigated factors (age, comorbidity, vital signs (respiratory rate, oxygen saturation, heart rate, temperature, systolic blood pressure, diastolic blood pressure, Glasgow Coma Scale) and laboratory values (haemoglobin, white blood cell count (WBC), bands, creatinine, C-reactive protein, $\mathrm{pH}, \mathrm{pCO} 2, \mathrm{pO} 2$, bicarbonate, lactate)) that could have influenced the discordance.

\section{Statistics}

All data were analysed using IBM SPSS Statistics 20.0. for Windows (IBM, Armont, New York, USA, 2011) and MedCalc for Windows, version 12.4.0.0 (MedCalc Software, Mariakerke, Belgium). Descriptive analyses were made to analyse the baseline characteristics and fulfilment of the SIRS criteria and the sepsis definition. All percentages were valid percentages. The Chi-square or Fisher's exact tests as appropriate were used to test for differences in proportions. Means and medians were compared with the independent T-test and Mann-Whitney-U-test, respectively. Mortality rates were analysed using Kaplan-Meier survival tests and compared using the log-rank test. Differences between the AUCs were calculated using the DeLong test [18]. All p-values $<0.05$ were considered significant.

\section{Results}

\section{Study population}

In the study period, 587 patients were assessed by internists at the ED and subsequently admitted to the hospital. In total, 23 patients were excluded because they were admitted to other departments and another 26 because of revisits or readmissions. In total, 538 patients with a mean age of 61.5 years were included in our analysis (Table 1). Of these 538 patients, 264 (49.1\%) were diagnosed with or suspected to have an infection. An elevated heart rate and abnormal WBC were the two most prevalent SIRS criteria (40.7 and $38.1 \%$, respectively). In 72 (13.4\%) of 538 patients, one or more SIRS criteria were missing (and considered normal). Blood cultures were taken in 208 (38.7\%) patients; of these, 43 $(20.7 \%)$ were positive. Any evidence of infection, either in cultures and/or radiologic examinations, was found in 188 of 416 (45.2\%) patients.

\section{Patients with $<2$ and $\geq 2$ SIRS criteria}

Of all 538 patients, 181 (33.6\%) had presence of $\geq 2$ SIRS criteria; these patients were more often diagnosed with an infection than those with $<2$ SIRS criteria ( 76.8 vs. $35.0 \%, p<0.001$, Table 1). Evidence of infection from cultures and/or radiologic examinations was more often present in patients with $\geq 2$ SIRS criteria than in those with $<2$ SIRS criteria ( 55.2 vs. $24.6 \%, p<0.001)$. Mortality, either in-hospital, within 28 days or within one year, was higher in those with $\geq 2$ SIRS criteria $(12.7,16.6$ and $34.3 \%$, respectively) than in those with $<2$ SIRS criteria $(4.2,5.0$ and $21.8 \%$, respectively, $\mathrm{p} \leq 0.001)$. 
Table 1 Patient characteristics

\begin{tabular}{|c|c|c|c|}
\hline N (\%), mean (SD) or median (IQR) & $\begin{array}{l}\text { All patients } \\
\mathrm{N}=538\end{array}$ & $\begin{array}{l}<2 \text { SIRS criteria } \\
\mathrm{N}=357(66.4)\end{array}$ & $\begin{array}{l}\geq 2 \text { SIRS criteria } \\
\mathrm{N}=181(33.6)\end{array}$ \\
\hline \multicolumn{4}{|l|}{ Demographics } \\
\hline Age (years) & $61.5(19.0)$ & $60.7(19.5)$ & $63.1(18.0)$ \\
\hline Male & $253(47.0)$ & $159(44.5)$ & $94(51.9)$ \\
\hline \multicolumn{4}{|l|}{ Vital parameters } \\
\hline Respiratory rate (breaths/min) & $15.7(4.6)$ & $14.3(2.0)$ & $18.2(6.8)$ \\
\hline Heart rate (beats/min) & $90.0(20.9)$ & $81.8(16.1)$ & $105.4(20.2)$ \\
\hline Temperature $\left({ }^{\circ} \mathrm{C}\right)$ & $37.2(1.1)$ & $36.9(0.7)$ & $37.6(1.4)$ \\
\hline \multicolumn{4}{|l|}{ Laboratory values } \\
\hline WBC (10E9/L) & $9.4(6.8-13.4)$ & $8.7(6.8-11.4)$ & $12.8(6.4-16.9)$ \\
\hline $\mathrm{CRP}(\mathrm{mg} / \mathrm{L})$ & $35(7-114)$ & $19.0(4-70)$ & $85.0(23-212)^{*}$ \\
\hline Lactate (mmol/L) & $2.0(1.4-2.8)$ & $1.6(1.3-2.3)$ & $2.2(1.5-3.4)^{\ddagger}$ \\
\hline \multicolumn{4}{|l|}{ Presence of SIRS criteria } \\
\hline Respiratory rate/pCO2 & $81(15.1)$ & $13(3.6)$ & $68(37.6)$ \\
\hline Heart rate & $219(40.7)$ & $69(19.3)$ & $150(82.9)$ \\
\hline Temperature & $103(19.1)$ & $22(6.2)$ & $81(44.8)$ \\
\hline WBC/bands & $205(38.1)$ & $73(20.4)$ & $132(72.9)$ \\
\hline Suspected/proven infection & $264(49.1)$ & $125(35.0)$ & $139(76.8)^{*}$ \\
\hline \multicolumn{4}{|l|}{ Evidence of infection } \\
\hline Any culture $(n=292)$ : positive & $123(42.1)$ & $59(39.6)$ & $64(44.8)^{*}$ \\
\hline Blood culture $(n=208)$ : positive & $43(20.7)$ & $16(18.0)$ & $27(22.7)^{*}$ \\
\hline $\begin{array}{l}\text { Radiologic examination }(n=381) \text { : } \\
\text { positive }\end{array}$ & $110(28.9)$ & $43(19.7)$ & $67(41.1)^{*}$ \\
\hline $\begin{array}{l}\text { Cultures and/or radiologic } \\
\text { examinations ( } n=416) \text { : positive }\end{array}$ & $188(45.2)$ & $88(35.2)$ & $100(60.3)^{*}$ \\
\hline \multicolumn{4}{|l|}{ Comorbidity } \\
\hline Charlson Index & $2(0-4)$ & $2(0-4)$ & $2(0-4)^{\ddagger 1}$ \\
\hline \multicolumn{4}{|l|}{ Mortality } \\
\hline In-hospital & $38(7.1)$ & $15(4.2)$ & $23(12.7)^{*}$ \\
\hline Within 28 days & $48(8.9)$ & $18(5.0)$ & $30(16.6)^{*}$ \\
\hline Within 1 year & $141(26.2)$ & $78(21.8)$ & $62(34.3)^{\#}$ \\
\hline \multicolumn{4}{|c|}{$\begin{array}{l}\text { SD = standard deviation; IQR = interquartile range; } \mathrm{SIRS}=\text { Systemic Inflammatory Response Syndrome; WBC } \\
=\text { white blood cell count; } \mathrm{CRP}=\mathrm{C} \text { - reactive protein. All percentages are valid percentages. } \\
{ }^{*} \mathrm{p}<0.001 ;{ }^{\ddagger} p<0.05 ;{ }^{*} \mathrm{p}=0.001 \text { (only } p \text { values below } 0.05 \text { were depicted); }{ }^{\ddagger} \text { higher in the group with } \geq 2 \text { SIRS } \\
\text { criteria. }\end{array}$} \\
\hline
\end{tabular}




\section{Diagnostic value}

Of all 538 patients, 139 (25.8\%) patients fulfilled the sepsis definition, 114 patients (21.2\%) the face diagnosis and 113 patients (21.0\%) the discharge diagnosis sepsis. The sepsis definition had a sensitivity of $73.5 \%$ and a specificity of $86.8 \%$, with a corresponding AUC of 0.80 (95\% $\mathrm{Cl} 0.75-0.85)$, when compared with the discharge diagnosis (Table 2). The face diagnosis had a sensitivity of $81.4 \%$, a specificity of $94.8 \%$ and had a significantly higher AUC of $0.88(95 \% \mathrm{Cl} 0.84-0.93)$ than the sepsis definition ( $p<0.001$, Table 2$)$. 'Sepsis by sepsis definition and/or face diagnosis' had a higher AUC of 0.86 (95\%CI0.82-0.90) compared to the sepsis definition alone, ' $\geq 2$ SIRS' and 'suspected/proven infection' (AUC 0.80, 0.76 and 0.78 , respectively $(p<0.001))$.

Table 2 Diagnostic value of the sepsis definition and several other tests compared with the discharge diagnosis (reference test) in all patients and in the subgroup with suspected or proven infection

\begin{tabular}{|c|c|c|c|c|c|}
\hline$\%$ & Sensitivity & Specificity & PPV & NPV & $\operatorname{AUC}(95 \% \mathrm{Cl})$ \\
\hline \multicolumn{6}{|l|}{ All Patients $(\mathrm{N}=538)$} \\
\hline Sepsis definition & 73.5 & 86.8 & 59.7 & 92.5 & $0.80(0.75-0.85)^{n}$ \\
\hline Face diagnosis & 81.4 & 94.8 & 80.7 & 95.0 & $0.88(0.84-0.93)^{*}$ \\
\hline $\begin{array}{l}\text { Sepsis by sepsis definition } \\
\text { and/or face diagnosis }\end{array}$ & 88.4 & 84.3 & 59.6 & 96.5 & $0.86(0.82-0.90)^{*}$ \\
\hline$\geq 2$ SIRS & 75.2 & 77.4 & 47.0 & 92.2 & $0.76(0.71-0.82)$ \\
\hline Suspected/proven infection & 93.8 & 62.8 & 40.2 & 97.5 & $0.78(0.74-0.83)$ \\
\hline \multicolumn{6}{|c|}{ Subgroup of patients with suspected/proven infection ( $N=264)$} \\
\hline$\geq 1$ SIRS & 97.2 & 26.6 & 47.0 & 93.3 & $0.62(0.55-0.69)$ \\
\hline$\geq 2$ SIRS & 78.3 & 64.6 & 59.7 & 81.6 & $0.71(0.65-0.78)^{1}$ \\
\hline$\geq 3$ SIRS & 37.7 & 91.8 & 75.5 & 68.7 & $0.65(0.58-0.72)$ \\
\hline 4 SIRS & 7.5 & 99.4 & 88.9 & 61.6 & $0.67(0.60-0.74)^{2}$ \\
\hline$\geq 1$ SIRS (vital signs only) & 86.8 & 47.5 & 52.6 & 84.3 & $0.67(0.61-0.74)^{3}$ \\
\hline$\geq 2$ SIRS (vital signs only) & 51.9 & 88.0 & 74.3 & 73.2 & $0.70(0.63-0.77)^{3}$ \\
\hline 3 SIRS (vital signs only) & 11.3 & 97.5 & 75.0 & 62.1 & $0.54(0.47-0.62)$ \\
\hline \multicolumn{6}{|c|}{$\begin{array}{l}\text { SIRS = systemic inflammatory response syndrome criteria; } \mathrm{PPV}=\text { positive predicting value; NPV = negative } \\
\text { predicting value; AUC = area under the ROC-curve; } 95 \% \mathrm{Cl}=95 \% \text { confidence interval. } \\
\text { " } p<0.001 \text { Compared to } \geq 2 \mathrm{SIRS} \text { (all patients); }{ }^{*} p<0.001 \mathrm{Compared} \text { to sepsis definition, } \geq 2 \mathrm{SIRS} \text {, suspected/ } \\
\text { proven infection (all patients); }{ }^{1} p<0.001 \text { Higher diagnostic value compared to } \geq 1 \mathrm{SIRS}, \geq 3 \mathrm{SIRS} \text { and } 4 \text { SIRS } \\
\text { (subgroup); }{ }^{2} p<0.001 \text { Higher diagnostic value compared to } \geq 1 \text { and } \geq 3 \text { SIRS (subgroup); }{ }^{3} p<0.001 \text { Higher } \\
\text { diagnostic value compared to } 3 \text { SIRS (subgroup). }\end{array}$} \\
\hline
\end{tabular}




\section{Subgroup of patients with suspected or proven infection}

In the subgroup of patients with suspected/proven infection, the test ' $\geq 2$ SIRS criteria' had higher diagnostic value (AUC $0.71(95 \% \mathrm{Cl} 0.65-0.78$ ), $p<0.001$ ) than $\geq 1, \geq 3$ or 4 SIRS criteria (Table 2). Although the sensitivity of the sepsis definition (i.e. $\geq 2$ SIRS criteria) was higher in the subgroup of patients with an infection than in the whole study population, the specificity was lower, resulting in a lower AUC. The highest AUC of SIRS criteria consisting of vital signs only was found when $\geq 2$ SIRS were positive (AUC $0.70(95 \% \mathrm{Cl} 0.63-0.77)$ ).

\section{The diagnosis sepsis over time}

In total, 139 (25.8\%) of 538 patients fulfilled the sepsis definition at the ED, 114 (21.2\%) received the face diagnosis sepsis, and $113(21.0 \%)$ patients were eventually diagnosed with sepsis at discharge (Figure 1 and 2). However, analysis of the diagnosis over time showed that these numbers do not concern the same patients. In total, 75 (of 139; 54.0\%) patients fulfilled the sepsis definition at the ED and received both the face and discharge diagnosis. However, $56(44+12 / 139 ; 40.3 \%)$ patients who fulfilled the sepsis definition did not get the discharge diagnosis of sepsis. Of these 56 patients, $44(78.6 \%)$ had a correct face diagnosis at the ED as not having sepsis. Of the 399 patients who did not fulfil the definition of sepsis, 369 (359+10; 92.5\%) patients eventually got the discharge diagnosis of'no sepsis'. However, $30(17+13$ of $399 ; 7.5 \%)$ were missed as having sepsis by the sepsis definition. In total, during ED and hospital stay, $104(27+52+13+12 ; 19.3 \%)$ patients crossed over once or twice between the diagnosis 'sepsis' and 'no sepsis'.

Factors that could explain discordance between the sepsis definition and discharge diagnosis

There were no significant differences in age, comorbidity, vital signs and laboratory values between the patients who received the sepsis definition but not the discharge diagnosis $(44+12=56)$ or vice-versa $(17+13=30)($ Table 3$)$. 
Chapter 2

Figure 1 Diagnosis from the sepsis definition at the ED to the discharge diagnosis

Sepsis definition

$\geq 2$ SIRS and infection
Face diagnosis sepsis
Discharge diagnosis

sepsis

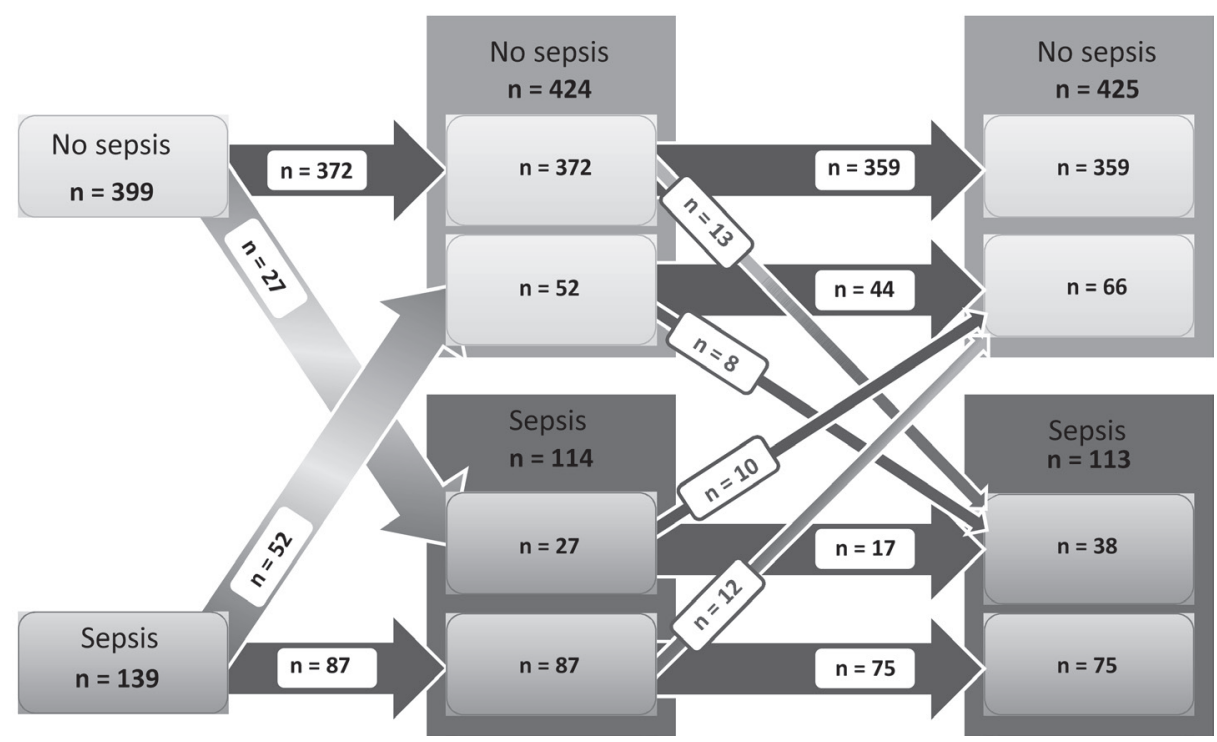

Figure 2 Number of patients with sepsis or no sepsis according to the sepsis definition, face diagnosis and discharge diagnosis (reference test)
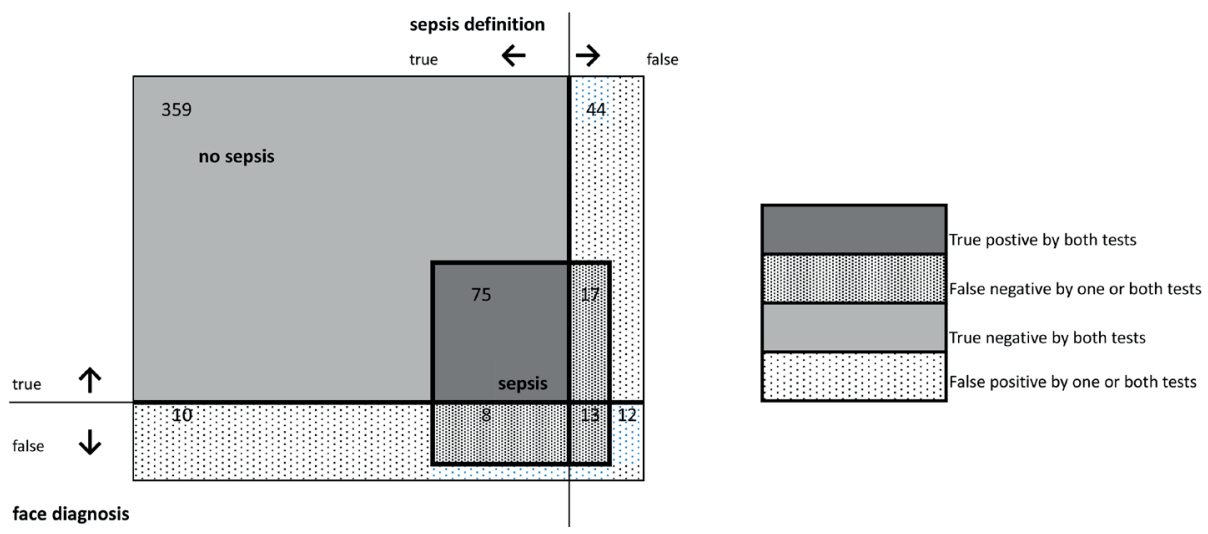
Table 3 Characteristics of the patients with discordance between the sepsis definition and the discharge diagnosis sepsis

\begin{tabular}{|c|c|c|}
\hline Mean (SD) or median (IQR) & $\begin{array}{l}\text { Sepsis definition: } \\
\text { sepsis } \\
\text { Discharge diagnosis: } \\
\text { no sepsis } \\
\mathrm{N}=56\end{array}$ & $\begin{array}{l}\text { Sepsis definition: } \\
\text { no sepsis } \\
\text { Discharge diagnosis: } \\
\text { sepsis } \\
\mathrm{N}=30\end{array}$ \\
\hline Age (years) & $66.5(52.8-77)$ & $66.5(58.0-75.0)$ \\
\hline Charlson index & $2.0(1-4)$ & $2.5(1-4)$ \\
\hline \multicolumn{3}{|l|}{ Vital parameters } \\
\hline Respiratory rate (breaths/min) & $14(14-23)$ & $14(14-14)$ \\
\hline Oxygen saturation (\%) & 97 (94-99) & 97 (94-99) \\
\hline Heart rate (beats/min) & $99.3(19.7)$ & $85.8(16.3)$ \\
\hline Temperature $\left({ }^{\circ} \mathrm{C}\right)$ & $37.5(1.4)$ & $37.2(0.9)$ \\
\hline Systolic blood pressure $(\mathrm{mmHg})$ & $125(110-135)$ & $120(100-140)$ \\
\hline Diastolic blood pressure $(\mathrm{mmHg})$ & $70(60-80)$ & $70(55-80)$ \\
\hline Glasgow Coma Score & $15(15-15)$ & $15(15-15)$ \\
\hline \multicolumn{3}{|l|}{ Laboratory values } \\
\hline Haemoglobin (mmol/L) & $7.6(1.7)$ & $7.5(1.4)$ \\
\hline WBC (x10E9/L) & $12.9(8.4-18.1)$ & $11.8(7.7-16.1)$ \\
\hline Bands (\%) & $0(0-0.8)$ & $0(0-0)$ \\
\hline Creatinine (umol/L) & $97.0(77.0-148.0)$ & $99.5(76.8-152.8)$ \\
\hline C-reactive protein (mg/L) & $78.0(26.0-229.0)$ & $70.0(45.5-259.8)$ \\
\hline $\mathrm{pH}$ & $7.45(0.1)$ & $7.42(0.1)$ \\
\hline $\mathrm{pCO} 2(\mathrm{kPa})$ & $4.7(2.1)$ & $4.7(0.9)$ \\
\hline $\mathrm{pO} 2(\mathrm{kPa})$ & $9.4(2.5)$ & $8.7(2.3)$ \\
\hline Bicarbonate $(\mathrm{mmol} / \mathrm{L})$ & $22.9(5.8)$ & $22.3(4.6)$ \\
\hline Lactate $(\mathrm{mmol} / \mathrm{L})$ & $1.7(0.8)$ & $1.6(0.4)$ \\
\hline
\end{tabular}

\section{Discussion}

In this study, we found that almost half of the patients who visit the ED and are subsequently admitted to the internal medicine department are diagnosed with or suspected of having an infection. One third of the ED patients had $\geq 2$ SIRS criteria and of these, more than three quarters had an infection. In total, $25.8 \%$ of the patients fulfilled the sepsis definition ( $\geq 2$ SIRS criteria and suspected/proven infection), $21.2 \%$ the face diagnosis sepsis and $21.0 \%$ the discharge diagnosis sepsis. The sepsis definition had acceptable diagnostic value. This diagnostic value was however lower than the face diagnosis (diagnosis made by the ED physician). Over time, during stay at the ED and the hospital, 104 (19.3\%) patients crossed over once or twice between the diagnosis 'sepsis' and 'no sepsis'. In the end, $40.3 \%$ 
of the patients who fulfilled the sepsis definition were wrongly diagnosed as having sepsis. Compared to the discharge diagnosis, $7.5 \%$ of the sepsis patients were missed by the sepsis definition.

One third of our ED patients had $\geq 2$ SIRS criteria. These patients were more often diagnosed with an infection ( 76.8 vs. $35.0 \%$ ) and 28 day mortality was higher ( 16.6 vs. $5.0 \%$ ) than in those with $<2$ SIRS criteria. This difference in mortality was not seen in another study, where only $29.2 \%$ of the patients had $\geq 2$ SIRS criteria and which included patients with a suspected infection only [19]. Another study included patients with severe sepsis and found no association between the presence of SIRS (72\%) and development of severe sepsis or a critical course of disease. Therefore, the prognostic value of $\geq 2$ SIRS criteria is only present in a general ED population [14].

An important problem when studying the diagnostic value of the sepsis definition is that the definition is the gold standard and therefore by definition impossible to challenge. However, there are concerns about the diagnostic value of this gold standard [7, 9, 10, 19]. The criteria to diagnose sepsis must at least be highly sensitive in order not to miss sepsis. However, the criteria also need specificity because a false diagnosis of sepsis could lead to missing important other diagnoses that could be life-threatening, for example a pulmonary embolism. Low specificity could also lead to overtreatment resulting in an increase in bacterial resistance. High diagnostic value of the SIRS criteria is most important in a general ED population where immediate decisions should be made about diagnostic evaluation and treatment. Therefore, we conducted an analysis in a rather unselected (internal medicine) ED population.

Another problem when studying the diagnostic value of the sepsis definition is that over time, more information becomes available, which may influence the diagnostic value of the evaluated tests. Therefore, we will first discuss our findings on the classical tests regarding diagnostic value (sensitivity etc.), and then, we will focus on the diagnosis over time.

Indeed, we found that the sepsis definition only had a sensitivity of $73.5 \%$ and a specificity of $86.8 \%$. This means that over a quarter of the patients with sepsis were missed by the sepsis definition and more than $10 \%$ of the patients were wrongly diagnosed as having sepsis by the sepsis definition. Furthermore, the diagnostic value of the sepsis definition in patients at the ED is lower than in the ICU $[11,12,20]$. This is in accordance with another study that found similar sensitivity and even lower specificity of the sepsis definition in diagnosing severe sepsis and septic shock in patients with an infection at the ED [14]. Dividing the definition into two index tests: ' $\geq 2$ SIRS criteria' and 'presence of suspected/ proven infection', showed rather low diagnostic value. These two index tests should therefore not be used separately when screening for sepsis at the ED.

The face diagnosis had higher diagnostic value than the sepsis definition. We considered the comparison of the face diagnosis with the discharge diagnosis important because at the ED, important decisions on treatment and disposition have to be made, based on preliminary clinical assessments and test results. To make the face diagnosis at the ED, the ED physician probably combines the sepsis definition with pattern recognition (clinical impression, vital signs, laboratory results and/or radiologic examinations). This probably explains why the face diagnosis at the ED has higher diagnostic value than the sepsis defi- 
nition. Indeed, the combination 'sepsis by sepsis definition and/or face diagnosis' resulted in a higher diagnostic value than when the sepsis definition was used solely. This is in agreement with the decision of the International Sepsis Definitions Conference of 2001 not to change the sepsis definition, but to expand it with a list of signs and symptoms of sepsis to include pattern recognition [21]. However, this list consists of more than 20 items thereby limiting the usefulness both in research and daily practice. Another explanation for the high diagnostic value of the face diagnosis could be that the face diagnosis is immediately adopted as final discharge diagnosis at the ED and not challenged or revised anymore during the hospital stay. However, this is not very likely, because the results of cultures and/or radiologic examinations and observation of the patient in the hospital enables an accurate discharge diagnosis. In short, the face diagnosis is a black box because we do not know which factors contribute to the diagnosis sepsis. This might also be the reason why it is difficult to train physicians in the recognition of sepsis. Perhaps, especially for inexperienced clinicians, with an AUC of 0.80 , the sepsis definition can help to diagnose sepsis.

In the subgroup with a suspected/proven infection, the presence of $\geq 2$ SIRS criteria yielded the highest, but only moderate diagnostic value (AUC 0.71). The same is true for the diagnostic value of $\geq 2$ SIRS criteria consisting of the vital signs only (heart rate, respiratory rate, body temperature; AUC 0.70).This is in accordance with another study in which emergency medical services providers could identify patients with severe sepsis with a sensitivity of only $48 \%$ when at least two of three SIRS criteria were positive [22]. Despite limited sensitivity of SIRS criteria consisting of vital signs, this set of criteria could be a handy tool to make a first assessment during emergency medical transport by ambulance by raising awareness on the presence of a serious disease. This could lead to an earlier start of treatment at the ED.

In the present study, we found a high discordance rate between the sepsis definition, face diagnosis and discharge diagnosis. Therefore, when the diagnosis over time is taken into account, the diagnostic value of the sepsis definition is less than calculated, due to cross-over between the groups, which occurred in $19.3 \%$ of the patients.

Of all patients who were not diagnosed with sepsis by the definition, $7.5 \%$ were diagnosed at discharge as having sepsis. Fortunately, more than half of the patients who were missed by the sepsis definition, received the face diagnosis of sepsis at the ED. In addition, $40.3 \%$ of the patients did fulfil the sepsis definition, but did not get the discharge diagnosis. Of these patients, $78.6 \%$ had a correct face diagnosis at the ED as not having sepsis, which illustrates that the diagnosis sepsis made by the sepsis definition is often adjusted by the ED physician. It is possible that the experience of the physician or more detailed information on the clinical presentation and history of the patient contribute to the face and final discharge diagnosis. However, we were not able to identify factors that could explain the inconsistency between the sepsis definition and the discharge diagnosis. We found no differences in age, Charlson Comorbidity Index, vital signs nor simple blood tests.

There are some limitations in the present study that need to be addressed. First, we assessed the patients' charts retrospectively. This increases the chance of selection bias. However, our charts are rather complete and include information from doctors, nurses, ancillary investigations and data from the municipal administration (regarding mortality) as well. Second, in case of missing SIRS criteria, we deduced these from the description of the 
patient at the moment of presentation at the ED, which could induce misclassification bias. Missing SIRS criteria is to be expected in patients with for example an intoxication. In such a case, it is not mandatory to measure a white blood cell count. Third, by retrieving a clinical diagnosis retrospectively, the face diagnosis and discharge diagnosis could be biased by knowledge on the presence of SIRS criteria and/or clinical outcome. We tried to minimize this problem by assessing the face diagnosis and discharge diagnosis by two investigators separately. A fourth issue might be that we made the assumption that the clinical discharge diagnosis is the most reliable tool to define sepsis. This is of course open for debate. However, there are no definitive ways to challenge the sepsis definition. Last, the face diagnosis is a black box. It is probable that our physicians are (proportionally) more often exposed to sepsis than doctors in other countries as about a quarter of the patients presenting at our ED fulfil the sepsis definition. There are two reasons for this high exposure: first, most patients at our ED have been referred by a general practitioner to the internist and are therefore more severely ill $[14,23,24]$ and second, this study was performed in a secondary and tertiary teaching hospital, with a substantial number of (immune compromised) septic patients and where more physicians per patient are involved. This high exposure to sepsis patients could be beneficial to the recognition of sepsis at the ED (face diagnosis) and explain the relatively lower diagnostic value of the sepsis definition.

\section{Future perspectives}

The diagnostic value of the current sepsis criteria is not perfect and clinical judgment of the ED physician remains very important. Recently, the diagnostic value of several biomarkers in diagnosing sepsis was studied. However, until now, separately, none of these appear to have high value in diagnosing sepsis [25]. In the future, the diagnosis of sepsis will be made by a combination of training of physicians in the recognition of sepsis, vital signs, simple laboratory results and one or more biomarkers [26]. It is also possible that a new set of diagnostic criteria aimed at the recognition of sepsis specifically at the ED would result in better and earlier recognition of sepsis.

\section{Conclusion}

The sepsis definition (presence of $\geq 2$ SIRS criteria and a proven/suspected infection) had less diagnostic value than the face diagnosis (diagnosis by the ED physician) in a general medical population at the ED, when the discharge diagnosis was used as reference. During stay at the ED and the hospital, many (19.3\%) patients crossed over from 'sepsis' to 'no sepsis' or vice versa. Therefore, good clinical judgment remains a prerequisite for diagnosing sepsis correctly. 


\section{References}

1. Dombrovskiy VY, Martin AA, Sunderram J, Paz HL. Rapid increase in hospitalization and mortality rates for severe sepsis in the United States: a trend analysis from 1993 to 2003 . Crit Care Med. 2007;35(5):1244-50.

2. Angus DC, van der Poll T. Severe sepsis and septic shock. N Engl J Med. 2013;369(21):2063.

3. Seymour CW, Rea TD, Kahn JM, Walkey AJ, Yealy DM, Angus DC. Severe sepsis in pre-hospital emergency care: analysis of incidence, care, and outcome. Am J Respir Crit Care Med. 2012;186(12):126471.

4. Rivers EP, Katranji M, Jaehne KA, Brown S, Abou Dagher G, Cannon C, et al. Early interventions in severe sepsis and septic shock: a review of the evidence one decade later. Minerva Anestesiol. 2012;78(6):712-24.

5. Kumar A, Roberts D, Wood KE, Light B, Parrillo JE, Sharma S, et al. Duration of hypotension before initiation of effective antimicrobial therapy is the critical determinant of survival in human septic shock. Crit Care Med. 2006;34(6):1589-96.

6. Bone RC, Sibbald WJ, Sprung CL. The ACCP-SCCM consensus conference on sepsis and organ failure. Chest. 1992;101(6):1481-3.

7. Balk RA. Systemic inflammatory response syndrome (SIRS): where did it come from and is it still relevant today? Virulence. 2014;5(1):20-6.

8. Vincent JL, Abraham E, Annane D, Bernard G, Rivers E, Van den Berghe G. Reducing mortality in sepsis: new directions. Crit Care. 2002;6 Suppl 3:S1-18.

9. Klein Klouwenberg PM, Ong DS, Bonten MJ, Cremer OL. Classification of sepsis, severe sepsis and septic shock: the impact of minor variations in data capture and definition of SIRS criteria. Intensive Care Med. 2012;38(5):811-9.

10. Vincent JL. Dear SIRS, I'm sorry to say that I don't like you. Crit Care Med. 1997;25(2):372-4.

11. Alberti C, Brun-Buisson C, Burchardi H, Martin C, Goodman S, Artigas A, et al. Epidemiology of sepsis and infection in ICU patients from an international multicentre cohort study. Intensive Care Med. 2002;28(2):108-21.

12. Brun-Buisson C, Meshaka P, Pinton P, Vallet B, Group ES. EPISEPSIS: a reappraisal of the epidemiology and outcome of severe sepsis in French intensive care units. Intensive Care Med. 2004;30(4):580-8.

13. Jaimes F, Garces J, Cuervo J, Ramirez F, Ramirez J, Vargas A, et al. The systemic inflammatory response syndrome (SIRS) to identify infected patients in the emergency room. Intensive Care Med. 2003;29(8):1368-71.

14. Gille-Johnson P, Hansson KE, Gardlund B. Severe sepsis and systemic inflammatory response syndrome in emergency department patients with suspected severe infection. Scand J Infect Dis. 2013;45(3):186-93.

15. Thijssen WA, Giesen PH, Wensing M. Emergency departments in The Netherlands. Emerg Med J. 2012;29(1):6-9.

16. Needham DM, Scales DC, Laupacis A, Pronovost PJ. A systematic review of the Charlson comorbidity index using Canadian administrative databases: a perspective on risk adjustment in critical care research. J Crit Care. 2005;20(1):12-9.

17. Hilderink MJ, Roest AA, Hermans M, Keulemans YC, Stehouwer CD, Stassen PM. Predictive accuracy and feasibility of risk stratification scores for 28-day mortality of patients with sepsis in an emergency department. Eur J Emerg Med. 2014.

18. DeLong ER, DeLong DM, Clarke-Pearson DL. Comparing the areas under two or more correlated receiver operating characteristic curves: a nonparametric approach. Biometrics. 1988;44(3):837-45.

19. Vincent JL, Opal SM, Marshall JC, Tracey KJ. Sepsis definitions: time for change. Lancet. 2013;381(9868):774-5.

20. Kaukonen KM, Bailey M, Pilcher D, Cooper DJ, Bellomo R. Systemic inflammatory response syndrome criteria in defining severe sepsis. N Engl J Med. 2015;372(17):1629-38.

21. Levy MM, Fink MP, Marshall JC, Abraham E, Angus D, Cook D, et al. 2001 SCCM/ESICM/ACCP/ATS/SIS International Sepsis Definitions Conference. Crit Care Med. 2003;31(4):1250-6.

22. Guerra WF, Mayfield TR, Meyers MS, Clouatre AE, Riccio JC. Early detection and treatment of patients with severe sepsis by prehospital personnel. J Emerg Med. 2013;44(6):1116-25. 
23. Cildir E, Bulut M, Akalin H, Kocabas E, Ocakoglu G, Aydin SA. Evaluation of the modified MEDS, MEWS score and Charlson comorbidity index in patients with community acquired sepsis in the emergency department. Intern Emerg Med. 2013;8(3):255-60.

24. Geier F, Popp S, Greve Y, Achterberg A, Glockner E, Ziegler R, et al. Severity illness scoring systems for early identification and prediction of in-hospital mortality in patients with suspected sepsis presenting to the emergency department. Wien Klin Wochenschr. 2013;125(17-18):508-15.

25. Kibe S, Adams K, Barlow G. Diagnostic and prognostic biomarkers of sepsis in critical care. J Antimicrob Chemother. 2011;66 Suppl 2:ii33-40.

26. Gille-Johnson P, Hansson KE, Gardlund B. Clinical and laboratory variables identifying bacterial infection and bacteraemia in the emergency department. Scand J Infect Dis. 2012;44(10):745-52. 


\section{Section II}

Prehospital care in sepsis patients 



\section{Chapter 3}

\section{Septic patients arriving with Emergency Medical Services: a seriously ill population}

Maaike Groenewoudt*, Asselina A. Roest*, Farah Leijten, Patricia M. Stassen (* both authors contributed equally) 


\begin{abstract}
Objective

Sepsis is a serious condition with high mortality. Early treatment improves outcome and can be started by Emergency Medical Services (EMS) personnel.

The primary aim of our study was to investigate how many sepsis patients are transported by EMS to the internist at the emergency department (ED). The secondary aims were to compare these EMS patients with patients who arrived at the ED otherwise and to investigate how these patients were managed. We further investigated how often the diagnosis sepsis/infection was documented by EMS.
\end{abstract}

\title{
Methods
}

We retrospectively retrieved all ED and EMS data of patients with sepsis who were assessed by the internist between March 2011 and March 2012

\section{Results}

Half (48.0\%) of 654 sepsis patients were transported by EMS. These patients were more seriously ill (more severe grades of sepsis, more admittances to the hospital/intensive care unit) than patients who were transported otherwise. Mortality within 28 days was $19.4 \%$ compared to $6.5 \%$ in the other patients. Nevertheless, half of the EMS transports were considered not urgent, even in $34.6 \%$ of the patients with septic shock. Assessment of vital signs was not routinely performed and treatment was started in only $43.6 \%$. The diagnosis sepsis/infection was documented in $63.4 \%$.

\section{Conclusion}

Half of the patients with sepsis arrive at the ED by EMS. These patients are seriously ill, and although these patients are likely to benefit from early treatment, they are often transported with non-urgent rides and both assessment of vital signs and early start of treatment are not routinely performed. 


\section{Introduction}

Sepsis is a serious condition, leading to substantial morbidity and mortality [1]. Although increasing the awareness of the high prevalence and serious nature of sepsis has received much attention, this issue is still a challenge. After introduction of the Surviving Sepsis Campaign, which aims to improve the awareness of doctors dealing with sepsis and to provide treatment protocols, mortality decreased up to $17 \%[2,3]$.

The guidelines for treatment provided by the Surviving Sepsis Campaign address the treatment of the septic patient after arrival at the hospital and not during the pre-hospital phase [4]. This focus on the hospital phase leaves room to improve the recognition and initiation of treatment of the septic patient in the pre-hospital phase. In the Dutch treatment guideline of the Emergency Medical Services (EMS), a protocol for sepsis exists [5]. This guideline prescribes the administration of oxygen and intravenous fluids by the paramedic, if systolic blood pressure is below $90 \mathrm{~mm} \mathrm{Hg}$.

There are a few studies concerning the care of sepsis patients by EMS personnel. In the United States of America, patients with sepsis who were transported by ambulances were more seriously ill, and had a higher chance of dying than patients who arrived at the emergency department (ED) otherwise $[6,7,8]$. Interestingly, these EMS patients were treated earlier, not only by EMS personnel, but also during stay at the ED [6]. Thus, transportation by EMS not only offers an opportunity to treat septic patients early in the ambulance, but also accelerates the treatment after arrival at the hospital. These advantages for ambulance patients can, however, only be achieved when these patients are transported by EMS and when sepsis is recognized in the ambulance.

The primary aim of our retrospective study is to investigate how many patients with sepsis, severe sepsis and septic shock, who are assessed and treated at the ED by internists, are transported by EMS. The secondary aim is to compare these EMS patients with those who are transported otherwise, regarding demographic data, hospitalization rate, sepsis severity, management and outcome. We further aim to investigate whether sepsis is documented by EMS personnel and to investigate whether assessment, treatment and outcome differ between the patients who were documented to have sepsis by EMS personnel and those who were not.

\section{Methods}

\section{Design and setting}

This retrospective cohort study was performed in the Maastricht University Medical Centre (MUMC+). This hospital is a secondary and tertiary teaching hospital in the Netherlands. Yearly, approximately 22,750 patients visit our ED, of whom approximately 5,000 are assessed and treated by acute internists and their residents. At our ED, there are no emergency physicians and residents of clinical specialties, in our study acute internists, assess all patients who are referred to the ED. Most patients are referred by a general practitioner and almost all acute admissions originate from the ED. 
EMS is provided by "regionale ambulancevoorziening Zuid-Limburg" (RAV-ZL). The EMS team consists of a trained EMS driver and a highly trained paramedic. As the Netherlands is a densely populated country, the EMS can transport (almost) all urgent patients to an ED within 45 minutes and non-urgent patients within 60 minutes. This study is approved by the Medical Ethics Committee of MUMC+.

\section{Population and data}

All adult patients who were assessed by the internist at the ED between March 2011 and March 2012 were evaluated by the researchers. The diagnosis sepsis and its' severity were established at the ED using data from the electronic ED and hospital chart by one investigator and were confirmed by a second. In case of doubt, a third investigator decided on this issue. Sepsis was defined using international criteria; suspicion of infection and 2 or more of 4 systemic inflammatory response syndrome (SIRS) criteria: 1 ) heart rate $>90 / \mathrm{min}$, 2) respiratory rate $>20 / \mathrm{min}$ or pCO2 $<4.3 \mathrm{kPa}, 3$ ) temperature $<36.0^{\circ} \mathrm{C}$ or $>38.3^{\circ} \mathrm{C}$, 4) leukocytes $<4 \times 109 / \mathrm{L}$ or $>12 \times 109 / \mathrm{L}$ or $>10 \%$ leukocyte bands) [9]. Sepsis severity was divided into three categories: 1) sepsis, 2) severe sepsis and 3) septic shock. Sepsis was defined as a suspected infection and at least two SIRS-criteria; severe sepsis as sepsis with organ dysfunction (e.g. lactic acidosis, oliguria, or acute alternation of mental status) or sepsis induced hypotension; septic shock as severe sepsis with persistent hypotension despite adequate fluid administration $[9,10,11]$. If a patient presented twice at our ED within 28 days, the second visit was excluded for we assumed that the second visit concerned the same disease episode. In addition, we excluded the patients for whom we could not retrieve the means of transportation or data on 28 day mortality.

From the patients charts, demographic data, vital signs at arrival at the hospital, laboratory values, diagnosis and focus of infection at admittance were retrieved. All-cause mortality within 28 days, both in- and out-hospital, was retrieved from the patient charts, and if necessary by contacting the general practitioner.

Of all patients, who were assessed by the internist for sepsis, we retrieved whether they were transported by EMS. From the electronic database of the EMS, we retrieved data on the urgency and reason of the ride, the vital signs and treatments started in the ambulance. We excluded the patients with insufficient EMS data (e.g. when a patient was transported by an EMS service from outside our own region). The assessment of urgency is made by the emergency medical dispatch centre of the EMS. The urgency can change during the ride; these data were not evaluated in our study. Urgency category A1 is the highest urgency category (life threatening situation), while A2 is ascribed to an urgent, but not immediately life threatening situation and category $B$ to non-urgent situations [12].

\section{Outcomes and analysis}

We assessed the number of patients with sepsis who were transported by EMS. In addition, we compared those who were transported by EMS with those who were transported otherwise regarding demographic data, admission rates to both general wards and me- 
dium care/intensive care units, length of hospital stay, all-cause mortality within 28 days and focus of infection.

We analysed whether the EMS paramedic assessed and treated patients more frequently with increasing sepsis severity. Further, we analysed whether the EMS paramedic had documented whether the patients had sepsis or an infection or not. We further compared the number of assessments and treatments performed and started during the EMS ride and compared 28 day mortality between the patients who were documented to have sepsis/ infection and those who were not.

Using IBM SPSS 20.0. for Windows, the independent samples T-test and Mann-Withney U-test was used to compare continuous data in 2 groups. Categorical data were analysed using the chi square and Fisher Exact test to compare proportions of groups, when appropriate. Kaplan Meier survival curves were created and differences between curves were tested using the log rank test. $P$ values lower than 0.05 were considered significant.

\section{Results}

In the study period, 704 patients were assessed by the internist at the ED and diagnosed with sepsis (Figure 1). Forty patients who were readmitted within 28 days were excluded. Two patients were excluded because of unknown means of transportation and 8 because of incomplete follow up. Of the remaining 654 patients, 314 patients $(48.0 \%)$ were transported by ambulance. Data on outcome were available for all EMS patients and data on the EMS ride were available for 287 of these EMS patients (91.4\%).

Figure 1 Flowchart of the study population

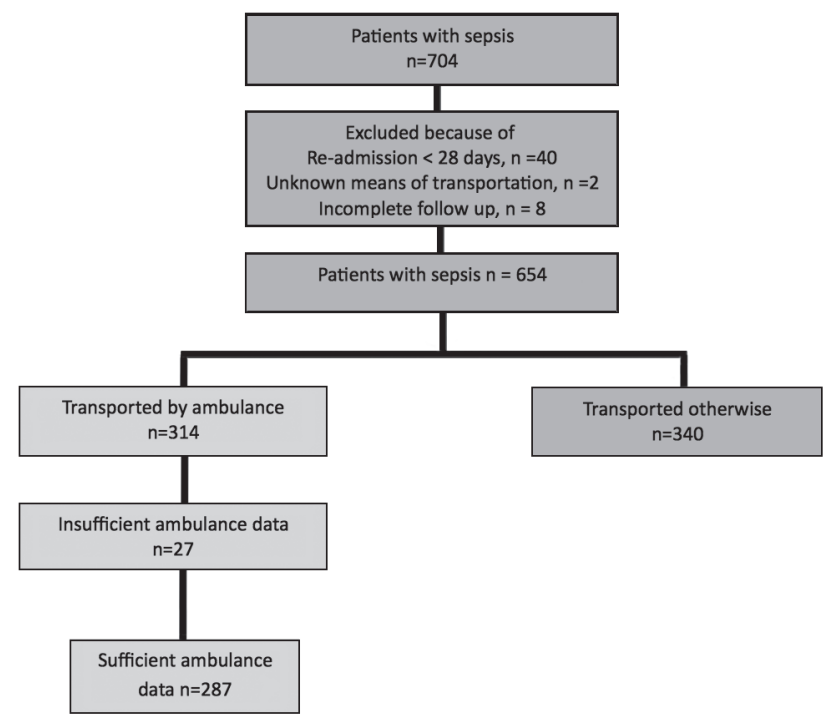


Table 1 shows the differences between the patients who were transported by EMS and those who were transported otherwise. The EMS patients were not only older (mean 71 vs. 56 years, $p<0.0001$ ), but also more seriously ill. The EMS patients were admitted more frequently to the hospital and more specifically to the ICU ( $p<0.0001$ for both comparisons). They also had more respiratory tract $(p<0.0001)$ and less hepatobiliary $(p=0.006)$ infections. EMS patients more frequently had severe sepsis or septic shock $(p<0.0001)$ and 28 -day mortality was higher $(19.4$ vs. $6.5 \%, p<0.0001)$ in this group.

Table 1 Patient characteristics

\begin{tabular}{|c|c|c|}
\hline Numbers (\%) & $\begin{array}{l}\text { Transported by } \\
\text { ambulance } \\
(n=314)\end{array}$ & $\begin{array}{l}\text { Transported otherwise } \\
(\mathrm{n}=340)\end{array}$ \\
\hline \multicolumn{3}{|l|}{ Demographics } \\
\hline Male & $149(47.5)$ & $155(45.6)$ \\
\hline Mean age (SD)* & $71 \pm 15$ & $56 \pm 16$ \\
\hline \multicolumn{3}{|l|}{ Outcome } \\
\hline Admittance to the hospital* & $307(97.8)$ & $292(85.9)$ \\
\hline Length of hospital stay (median, IQR)* & 7 (4-12) days & 5 (3-10) days \\
\hline MCU-admittance & $6(1.9)$ & $5(1.5)$ \\
\hline ICU-admittance* & $22(7.0)$ & $2(0.6)$ \\
\hline Mortality within 28 days* & $61(19.4)$ & $22(6.5)$ \\
\hline \multicolumn{3}{|l|}{ Severity of sepsis** } \\
\hline Sepsis & $136(43.3)$ & $263(77.4)$ \\
\hline Severe sepsis & $150(47.8)$ & $68(20.0)$ \\
\hline Septic shock & $28(8.9)$ & $9(2.6)$ \\
\hline \multicolumn{3}{|l|}{ Focus of infection } \\
\hline Respiratory* & $108(34.4)$ & $73(21.5)$ \\
\hline Urinary & $74(23.6)$ & $61(17.9)$ \\
\hline Gastrointestinal & $30(9.6)$ & 39 (11.5) \\
\hline Hepatobiliary* & $11(3.5)$ & $29(8.5)$ \\
\hline Skin/joint & $25(8.0)$ & $22(6.5)$ \\
\hline Other/unknown & $66(21.0)$ & $116(34.1)$ \\
\hline \multicolumn{3}{|c|}{$\begin{array}{l}\mathrm{SD}=\text { standard deviation; } I \mathrm{QR}=\text { inter quartile range. } \\
{ }^{*} \text { Significant difference between the two groups; }{ }^{* *} \text { Significant difference between the two groups regar- } \\
\text { ding the three grades of sepsis severity. }\end{array}$} \\
\hline
\end{tabular}

\section{Urgency of EMS transports}

Most EMS transports were judged non urgent (B category, 50.5\%), followed by A2 (32.4\%) and $\mathrm{A} 1$ (17.1\%, Table 2$)$. With increasing severity of sepsis, the transports were not judged as more urgent $(p=0.42)$. More than one third $(34.6 \%)$ of the patients with septic shock 
were transported with a B-ride. There was no significant difference in mortality between the EMS urgency categories (data not shown, $p=0.66$ ).

Table 2 Urgency of and assessment and treatment during EMS ride in relation to sepsis severity

\begin{tabular}{|c|c|c|c|c|}
\hline Numbers (\%) & $\begin{array}{l}\text { All patients } \\
(n=287)\end{array}$ & $\begin{array}{l}\text { Sepsis } \\
(n=118)\end{array}$ & $\begin{array}{l}\text { Severe sepsis } \\
(n=143)\end{array}$ & $\begin{array}{l}\text { Septic shock } \\
(n=26)\end{array}$ \\
\hline \multicolumn{5}{|l|}{ Urgency } \\
\hline A1-ride & $49(17.1)$ & $17(14.4)$ & $26(18.2)$ & $6(23.1)$ \\
\hline A2-ride & $93(32.4)$ & $36(30.5)$ & $46(32.2)$ & $11(42.3)$ \\
\hline B-ride & $145(50.5)$ & $65(55.1)$ & $71(49.7)$ & $9(34.6)$ \\
\hline \multicolumn{5}{|l|}{ Assessments } \\
\hline Blood pressure* & $192(66.9)$ & $75(63.6)$ & $93(65.0)$ & $24(92.3)^{\$}$ \\
\hline Heart rate & $116(40.4)$ & $48(40.7)$ & $52(36.4)$ & $16(61.5)$ \\
\hline Oxygen saturation & $119(41.5)$ & $46(39.0)$ & $59(41.3)$ & $14(53.8)$ \\
\hline Respiratory rate & $196(68.3)$ & $76(64.4)$ & $98(68.5)$ & $22(84.6)$ \\
\hline Mental state & $157(54.7)$ & $63(53.4)$ & $74(51.7)$ & 20 (76.9) \\
\hline Temperature & $68(23.7)$ & $29(24.6)$ & $32(22.4)$ & 7 (26.9) \\
\hline Five vital signs & $60(20.9)$ & $23(19.5)$ & $28(19.6)$ & $9(34.6)$ \\
\hline \multicolumn{5}{|l|}{ Treatment } \\
\hline Oxygen* & $102(35.5)$ & $29(24.6)^{\wedge \#}$ & $60(42.0)$ & $13(50.0)$ \\
\hline Iv fluids* & $68(23.7)$ & $24(20.3)^{\wedge}$ & $31(21.7)^{\ddagger}$ & $13(50.0)$ \\
\hline Oxygen and iv fluids* & $45(15.7)$ & $12(10.2)^{\wedge}$ & $23(16.1)^{\ddagger}$ & $10(38.5)$ \\
\hline
\end{tabular}

\section{Assessments and treatment during EMS transport}

Table 2 shows the assessments that were made during the EMS transport. The five most important vital signs (blood pressure, heart rate, oxygen saturation, respiratory rate and mental state) were assessed in $20.9 \%$ of all ambulance patients, while in $23.7 \%$ no vital signs were assessed.

Blood pressure was assessed more frequently in septic shock group than in the sepsis and the severe sepsis group $(p=0.015)$. Treatment was started by the EMS paramedic in $43.6 \%$ of the patients, while $45(15.7 \%)$ patients received both oxygen and intravenous fluids (Table 2). Patients with septic shock were treated more frequently with oxygen $(p=0.01)$, intravenous fluids $(p=0.004)$ or both $(p<0.0001)$ than patients with sepsis. Likewise, patients with septic shock received intravenous fluids $(p=0.002)$ and both oxygen and intravenous fluids $(p=0.008)$ more frequently than patients with severe sepsis. Patients with severe sepsis received more oxygen than those with sepsis $(p=0.003)$. 


\section{Documentation of sepsis by the EMS personnel}

Of the 287 EMS patients, 182 (63.4\%) were documented to have an infection, including the exact diagnosis sepsis in $31(10.8 \%$, Table 3$)$. The same percentages of documented infections $(67.9 \%)$ and sepsis $(13.6 \%)$ and were found in the 162 patients who were referred by a general practitioner as in the other patients $(p=0.34$ and 0.38 , respectively).

Blood pressure and mental state were assessed less frequently and the temperature was measured more frequently in the group with documented infection/sepsis $(p<0.05$ for the 3 comparisons). Treatment was similar as was mortality within 28 days in the 2 groups ( $p=0.17$, data not shown).

Table 3 Assessments, treatment and outcome in relation to documentation of sepsis and infection by EMS personnel

\begin{tabular}{|c|c|c|}
\hline Numbers (\%) & $\begin{array}{l}\text { Documentation of } \\
\text { infection/sepsis } \\
(n=182)\end{array}$ & $\begin{array}{l}\text { No documentation of } \\
\text { infection/sepsis } \\
\text { (n=105) }\end{array}$ \\
\hline \multicolumn{3}{|l|}{ Assessments } \\
\hline Blood pressure* & $114(62.6)$ & $78(74.3)$ \\
\hline Heart rate & $72(39.6)$ & $44(41.9)$ \\
\hline Oxygen saturation & $75(41.2)$ & $44(41.9)$ \\
\hline Respiratory rate & $117(64.3)$ & $79(75.2)$ \\
\hline Mental state* & $91(50.0)$ & $66(62.9)$ \\
\hline Temperature* & $50(27.5)$ & $18(17.1)$ \\
\hline Five vital signs ${ }^{\#}$ & $39(21.4)$ & $21(20.0)$ \\
\hline \multicolumn{3}{|l|}{ Treatment } \\
\hline Oxygen & $63(34.6)$ & $39(37.1)$ \\
\hline Iv fluids & $40(22.0)$ & $28(26.7)$ \\
\hline Oxygen and iv fluids & $28(15.4)$ & $17(16.2)$ \\
\hline \multicolumn{3}{|l|}{ Outcome } \\
\hline Mortality within 28 days & $31(17.0)$ & $26(24.8)$ \\
\hline
\end{tabular}

\section{Discussion}

Almost half of the patients with sepsis who are treated by internists at the ED are transported by EMS. These EMS patients are more seriously ill than those who are transported otherwise. The mortality rate of the septic EMS patients of $19.4 \%$ is high and much higher than those who are not transported by EMS (6.5\%).

The high mortality rate of sepsis we found was also seen in studies from the United States [8]. It is important to realize again that the mortality rate of our sepsis patients is 
higher than of many other diseases which are generally considered as extremely threatening. For instance, the mortality rate of acute myocardial infarction in men admitted to the hospital is $6.3 \%$ [13].

The vital signs of these sepsis patients were not routinely assessed and treatment was started in less than half (43.6\%) of the patients during the ambulance ride. Even with increasing sepsis severity, the number of patients in which vital signs were assessed did not increase, except for the blood pressure. It is remarkable that these assessments were not performed routinely. It is possible that the assessments were performed but not registered correctly, that the paramedic trusted on the assessments made by the general practitioner or a quick general assessment. It is not only important to assess vital signs to tailor and optimize treatment, but also to diagnose sepsis, as 3 of the vital signs are SIRS criteria (heart rate, respiratory rate, temperature). Measuring these vital signs will probably improve the recognition of sepsis.

We further found that our sepsis patients were not treated aggressively in the ambulance, as only $15.7 \%$ of the patients were treated with both oxygen and intravenous fluids and almost half of the patients received neither of these treatments, which is noted in other studies as well $[6,8]$. However, treatment was more frequently initiated with increasing sepsis severity. The registration of started treatments was probably rather reliable, for this registration is used for feedback on skills maintenance. Mortality was not significantly different between the patients who were documented as having infection or sepsis and those who were not. As treatment was similar between the documented and not documented groups, a difference in outcome cannot be expected. Early treatment in the ambulance could improve outcome if the results of early hospital treatment can be extrapolated to the pre-hospital phase. Further, improving treatment in the ambulance improves the care at the ED as patients who were treated with intravenous fluids during EMS transport received twice the volume of fluids in the first hour of ED stay $[7,8]$ and antibiotics were administered earlier if patients had been recognized as septic by the paramedic [6].

The diagnosis sepsis was documented in a minority (10.8\%) of the patients. Even if fever or infection were considered as documented sepsis, many cases of sepsis were not documented (36.6\%). Of course, we cannot conclude on the exact number of patients in which the diagnosis sepsis was recognized by the EMS staff for several reasons. First, we were only able to assess the cases of sepsis/infection that were documented in the electronic chart; it is possible that sepsis was recognized by the EMS paramedic more frequently. However, vital signs were not routinely assessed and most patients did not receive treatment, even those who turned out to have septic shock. Second, the diagnosis sepsis was established at the ED. It may be possible that sepsis was less serious or absent during the EMS transport, and therefore difficult to recognize. Third, one of the four SIRS criteria (leukocytes or leukocyte bands) can only be assessed at the ED, so it is possible that sepsis could not have been diagnosed in patients with only one other positive SIRS criterion. Although we cannot be precise on the exact number of missed diagnoses, we can at least conclude that the exact diagnosis sepsis was not documented in a majority of the EMS charts that were handed over to the ED. 
There are several reasons why the diagnosis sepsis was not documented by EMS personnel. It is possible that documentation of the diagnosis has no priority for the paramedic, as safe transportation of patients is more important. It is possible that because information is handed over verbally, the diagnosis is not written down as well, although this is obligatory. It is also possible that EMS personnel missed the diagnosis, for the paramedic used a global assessment instead of an exact assessment of the vital signs. The paramedic may not aim to diagnose sepsis specifically because treating life threatening symptoms is more important. It is also possible that the general practitioner missed the diagnosis as we found the same percentage documented sepsis (13.6\%) and suspected infections (67.9\%) in the group of patients who were referred by the general practitioner. The dispatcher at emergency medical dispatch centre may also have failed to recognize/document sepsis and thereby misled the paramedic, as almost half of the transports were considered non urgent $(50.5 \%)$.

Sepsis is a frequently missed diagnosis. EMS personnel missed up to $69 \%$ of those with a serious infection [14] and 52.2\% of patients with severe sepsis [15]. Hospital doctors in Brazil did not recognize severe sepsis in $43 \%$ [16] and in the United Kingdom, only $17 \%$ of the admitted patients with sepsis were documented to have sepsis [17]. Sepsis is apparently difficult to diagnose for more groups of health care professionals than EMS personnel [18], possibly resulting from a lack of knowledge, skills, tools or unclear rules and procedures.

Why is timely recognition of sepsis so important? First, the severity of sepsis tends to increase rapidly. More than $20 \%$ of patients with uncomplicated sepsis develop severe sepsis or septic shock within 72 hours [18]. Second, each hour of delay in the administration of antibiotics at the ED increased mortality with $7.6 \%$ in patients with severe sepsis [19]. Since the introduction of the Surviving Sepsis Campaign that advocates early treatment, absolute mortality in an international study has decreased by $6.2 \%$ [2], and in the Netherlands by $17 \%$ [3]. These data concern the care once the patient has reached the hospital.

Timely recognition and start of treatment remains a major challenge for professionals in the hospital, but apparently also for the whole chain of emergency care. Further prospective research concerning early recognition and treatment of sepsis by EMS personnel is necessary.

\section{Conclusion}

Almost half of the patients with sepsis who were assessed at the ED by the internist are transported by EMS. These patients are seriously ill as they are admitted more frequently to the hospital and the ICU and have more severe grades of sepsis than those who are transported otherwise. Mortality in the EMS patients is very high (19.4\%). However, assessment of vital signs and start of treatment are performed in only a minority of the patients and sepsis is not documented in a substantial part of the patients. Therefore, assessment of vital signs and acknowledgement of the serious nature of sepsis must be improved. 


\section{Acknowledgements}

$H$. Coenen, head of the emergency medical dispatch centre RAV-ZL,

N. Otten, ICT specialist emergency medical dispatch centre RAV-ZL,

J. Jansen, anaesthesiologist MUMC+, medical director of RAV-ZL

$G$. Vandergraesen, staff supporter of the emergency department MUMC+ 


\section{References}

1. Angus DC, Linde-Zwirble WT, Lidicker J, Clermont G, Carcillo J, Pinsky MR. Epidemiology of severe sepsis in the United States: analysis of incidence, outcome, and associated costs of care. Crit Care Med. 2001 Jul;29(7):1303-10.

2. Levy MM, Dellinger RP, Townsend SR, Linde-Zwirble WT, Marshall JC, Bion J, et al. The Surviving Sepsis Campaign: results of an international guideline-based performance improvement program targeting severe sepsis. Intensive Care Med. 2010 Feb;36(2):222-31.

3. Tromp M, Tjan DH, van Zanten AR, Gielen-Wijffels SE, Goekoop GJ, van den Boogaard M, et al. The effects of implementation of the Surviving Sepsis Campaign in the Netherlands. Neth J Med. 2011 Jun;69(6):292-8.

4. Dellinger RP, Carlet JM, Masur H, Gerlach H, Calandra T, Cohen J, et al. Surviving Sepsis Campaign guidelines for management of severe sepsis and septic shock. Crit Care Med. 2004 Mar;32(3):858-73.

5. Anonymous. [National protocol ambulance service, version 7.2.], 2011.

6. Studnek JR, Artho MR, Garner CL, Jones AE. The impact of emergency medical services on the ED care of severe sepsis. Am J Emerg Med. 2012 Jan;30(1):51-6.

7. Band RA, Gaieski DF, Hylton JH, Shofer FS, Goyal M, Meisal ZF. Arriving by emergency medical services improves time to treatment endpoints for patients with severe sepsis or septic shock. Acad Emerg Med. 2011 Sep;18(9):934-40.

8. Seymour CW, Cooke CR, Mikkelsen ME, Hylton J, Rea TD, Goss CH, et al. Out-of-hospital fluid in severe sepsis: effect on early resuscitation in the emergency department. Prehosp Emerg Care 2010 AprJun;14(2):145-52.

9. Levy MM, Fink MP, Marshall JC, Abraham E, Angus D, Cook D, et al. 2001 SCCM/ESICM/ACCP/ATS/SIS International Sepsis Definitions Conference. Crit Care Med. 2003 Apr;31(4):1250-6.

10. Dellinger RP, Levy MM, Rhodes A, Annane D, Gerlach H, Opal SM et al. Surviving Sepsis Campaign: International Guidelines for Management of Severe Sepsis and Septic Shock, 2012. Int Care Med. 2013; 39:165-228.

11. Bone RC, Balk RA, Cerra FB, Dellinger RP, Fein AM, Knaus WA, et al. Definitions for sepsis and organ failure and guidelines for the use of innovative therapies in sepsis. The ACCP/SCCM Consensus Conference Committee. American College of Chest Physicians/Society of Critical Care Medicine. Chest. 1992;101(6):1644-55.

12. Kommer GJ. [Ambulance service: how large is the market and is it increasing or decreasing?] RIVM; 2012; http://www.nationaalkompas.nl/zorg/ambulancezorg/vraag-gebruik-trend/[last entry 11-122012].

13. Schellekens J. Sepsis. National Compass Public Health] RIVM 2007. http://www.nationaalkompas.nl/ gezondheid-en-ziekte [last entry 11-12-2012].

14. Suffoletto B, Frisch A, Prabhu A, Kristan J, Guyette FX, Callaway CW. Prediction of serious infection during prehospital emergency care. Prehosp Emerg Care. 2011 Jul-Sep;15(3):325-30.

15. Guerra WF, Mayfield TR, Meyers MS, Cloatre AE, Riccio JC. Early detection and treatment of patients with severe sepsis bij prehospital personnel. J Emerg Med. 2013 Jun;44(6):1116-25.

16. Assuncao M, Akamine N, Cardoso GS, Mello PV, Teles JM, Nunes AL, et al. Survey on physicians' knowledge of sepsis: do they recognize it promptly? J Crit Care. 2010 Dec;25(4):545-52..

17. Cronshaw HL, Daniels R, Bleetman A, Joynes E, Sheils M. Impact of the Surviving Sepsis Campaign on the recognition and management of severe sepsis in the emergency department: are we failing? Emerg Med J. 2011 Aug;28(8):670-5.

18. Glickman SW, Cairns CB, Otero RM, Woods CW, Tsalik EL, Langley RJ, et al. Disease progression in hemodynamically stable patients presenting to the emergency department with sepsis. Acad Emerg Med. 2010 Apr;17(4):383-90.

19. Kumar A, Roberts D, Wood KE, Light B, Parrillo JE, Sharma S, et al. Duration of hypotension before initiation of effective antimicrobial therapy is the critical determinant of survival in human septic shock. Crit Care Med. 2006 Jun;34(6):1589-96. 


\section{Chapter 4}

\section{Ambulance patients with non-documented sepsis have a high mortality risk: \\ a retrospective study}

Asselina A. Roest*, Judith Stoffers*, Evelien Pijpers, Jochen Jansen, Patricia M. Stassen (* both authors contributed equally) 


\begin{abstract}
Objective

Sepsis is a serious disease leading to high mortality. Early recognition is important because treatment is most effective when started quickly.

The primary aim of this retrospective cohort study was to assess how many sepsis patients are documented as septic by ambulance staff. The secondary aims were to investigate how many sepsis patients are transported by ambulance, to compare them to patients transported otherwise, to investigate which factors influence documentation of sepsis and to assess whether documentation influences mortality.
\end{abstract}

\title{
Methods
}

We retrieved all data from ambulance and emergency department (ED) charts of patients who visited the internist at the ED from March 2011 to July 2012.

\section{Results}

In total, $47.4 \%$ ( $n=363$ ) sepsis patients were transported by ambulance. These patients were older (71.5 vs.55.7 years, $p<0.0001)$, admitted more frequently $(97.2$ vs.85.4\%, $\mathrm{p}<0.001)$, significantly more frequently had severe sepsis $(47.4 \mathrm{vs.} 25.8 \%, \mathrm{p}<0.0001)$ or septic shock ( 12.4 vs.4.0\%, p $<0.0001)$ and died more frequently within 28 days ( 17.9 vs.7.2\%, $p<0.0001$ ) than those who were transported otherwise.

In $41.9 \%$ of ambulance patients, sepsis was not documented by ambulance staff. Measurement of temperature was important for documentation of sepsis $(O R 11.2 ; 95 \% \mathrm{Cl} 5.2$ 24.4). In $32.1 \%$ of ambulance patients, sepsis could have been identified by assessing vital signs. Mortality in these non-documented patients was higher than in documented patients ( 25.7 vs. $12.9 \%, p=0.003)$.

\section{Conclusion}

Ambulance patients are seriously ill, but sepsis is often not documented by ambulance staff. Non-documentation is associated with high mortality and could be resolved by assessing vital signs, particularly the temperature. 


\section{Introduction}

\section{Background}

Sepsis is a serious disease with high mortality [1, 2]. Early start of treatment by administration of intravenous fluids and antibiotics at the emergency department (ED) reduces mortality up to $20 \%$ [3-9]. It is therefore important to ensure early recognition of patients with sepsis.

Treatment of severely ill patients is often initiated by ambulance staff [3-9]. The septic patient may also be treated in the ambulance if sepsis is recognized. Half of the sepsis patients are indeed transported by ambulance $[9,10]$ and these patients are often treated during the journey. Patients with severe sepsis were treated with intravenous fluids in $23-48 \%$ [10-13]. This treatment in the ambulance seems to decrease mortality and the number of intubations after arrival at the $\operatorname{ED}[12,13]$. However, only $48 \%$ of the patients with severe sepsis were recognized by ambulance staff even after a training that aimed to improve recognition of sepsis [14].Some of these patients were not recognized because laboratory values cannot be measured in the ambulance [14]. However, it is not clear why the other patients were not recognized, which factors influence recognition and whether recognition influences outcome of sepsis in the ambulance. We hypothesize that ambulance patients have a higher mortality risk than those transported otherwise and that recognition of sepsis in the ambulance influences this mortality in a positive way.

\section{Goals of this investigation}

The primary aim of this retrospective study is to assess how many sepsis patients are documented as having sepsis by ambulance staff. We, however, will first focus on our secondary aim: to assess how many patients with sepsis are transported by ambulance, to assess differences regarding age, sex, comorbidity, sepsis severity and mortality between these ambulance patients and patients who are transported otherwise, to assess which factors influence documentation of sepsis and to assess whether documentation influences 28day mortality.

\section{Methods}

\section{Study design and setting}

This retrospective cohort study was performed at the ED of the Maastricht University Medical Centre (MUMC+), a secondary and tertiary teaching hospital in the Netherlands with 715 beds. Acute internists and their residents assess all patients who are referred to the internist at the ED [15]. Annually, approximately 23,000 patients visit the ED, of whom 5500 are assessed by an internist. Of these patients, about 1500 patients are transported by ambulance. The ambulance team consists of a trained ambulance driver and a highly 
trained nurse. As the Netherlands is a densely populated country, the ambulance can transport all urgent patients to an ED within 45 minutes and non-urgent patients within 60 minutes. The ambulance is obliged to hand over an ambulance chart at the ED.

\section{Selection of participants}

We retrospectively retrieved data from the charts of all adult ( $\geq 18$ years) patients who presented to the internist between March 2011 and July 2012. All patients with the diagnosis sepsis, established at the ED, were included. Part of these patients ( $n=654 ; 75 \%$ ) have been included in another study in which we investigated which sepsis patients were transported by ambulance [16]. Patients were excluded when they revisited the ED within 28 days for we assumed that the second visit concerned the same disease episode. Other exclusion criteria were impossibility to access files, unclear means of transportation or incomplete follow-up (minimal follow-up was 28 days).

Sepsis was defined as a suspected infection and the presence of at least two SIRS (systemic inflammatory response syndrome) criteria $[17,18]$. The SIRS-criteria are defined as: Temperature $>38.3^{\circ} \mathrm{C}$ or $<36.0$; Heart rate $>90 /$ minute; Respiratory rate $>20 /$ minute or pCO2 $<4.3 \mathrm{kPa}$; Leukocytes $<4.10 \mathrm{E}^{9} / \mathrm{L}$ or $>12.10 \mathrm{E}^{9} / \mathrm{L}$ or $>10 \%$ leukocyte bands. Sepsis severity was divided into three categories: 1 ) sepsis, 2) severe sepsis and 3) septic shock [1, 2]. Severe sepsis was defined as sepsis with organ dysfunction (e.g. lactic acidosis, oliguria, or acute alternation of mental status) or sepsis induced hypotension; septic shock as severe sepsis with persistent hypotension despite adequate fluid administration $[17,18]$. The diagnosis sepsis and sepsis severity were established at the ED using data from the electronic $E D$ and hospital charts by one investigator and confirmed by a second. In case of doubt, a third investigator decided on the issue.

\section{Methods and measurements}

Of all included patients, we retrieved demographic data, means of transportation, assessments and laboratory values, treatment at the ED and outcome (admission to the hospital, length of ED and hospital stay and all-cause (in- and out-hospital) mortality within 28 days). In addition, severity of illness was assessed in two ways; according to the aforementioned three severity categories of sepsis and using the Modified Early Warning Score (MEWS) [19]. The MEWS is very feasible and frequently used in sepsis patients at the ED to assess severity of disease. $[20,21]$. The score consists of vital signs (respiratory rate, systolic blood pressure, temperature and level of consciousness) and is therefore also able to assess severity of illness in the ambulance. We used standardized abstraction forms to retrieve data from the charts. Fifteen percent of the charts were independently reviewed by a second investigator, to check the data. Items presented in categories or as text in the patient's charts were converted into absolute values (Appendix, Table 1).

For the patients who were transported by ambulance, additional data were retrieved from the electronic database of the ambulance services. These included diagnosis, vital signs, initiated treatment during the ambulance journey and urgency of the journey. The assessment of urgency level is made by the emergency medical dispatch centre. In this study, we divided the transports into urgent ( $A 1$ and $A 2$ ) journeys and non-urgent (B) jour- 
neys, because we hypothesized A1 and A2 journeys were equally time consuming. An A1 ambulance journey is advised when there is a life-threatening situation (with emergency lights), an A2 journey is advised when there is no immediate threat of life but direct presence of an ambulance is considered eligible. We could not retrieve data from transports from beyond our regional ambulance transport service $(n=29)$.

\section{Outcomes}

First, we retrieved how many patients were transported by ambulance and compared these to those who were transported otherwise, regarding age and sex, sepsis severity and outcome. In addition, we investigated whether age, means of transportation, sepsis severity (according to the three severity categories of sepsis and MEWS), the comorbidity malignancy, the use of cytotoxic and immune suppressive medication or length of ED stay independently influenced 28-day mortality.

The second part of our study focused on the ambulance patients. We retrieved which assessments were done and what treatment was started in the ambulance. We further retrieved in how many patients sepsis was documented. We considered sepsis documented when the terms "sepsis, infection and/or fever" were mentioned in the charts. We did two analyses to investigate which factors influence this documentation. We first compared "non-documented" patients with those who were documented to have sepsis regarding age, urgency of the journey, assessment of vital signs (heart rate, respiratory rate, temperature, oxygen saturation, mental state and blood pressure) and sepsis severity (according to the three severity categories of sepsis and the MEWS). Multivariate analysis was performed using Cox regression analysis to analyse which of these factors were independently associated with (presence or absence of) documentation. Second, we retrieved the presence of SIRS criteria in non-documented patients. We analysed whether the three SIRS criteria consisting of vital signs (heart and respiratory rate, temperature) were assessed and/ or were present during the ambulance ride. We did this to explore why patients were not documented to have sepsis in the ambulance and whether this was caused by the inability to assess the $\mathrm{pCO} 2$ or "leukocyte count or leukocyte bands" in the ambulance.

We further investigated whether treatment during the ambulance journey (i.e. intravenous fluids or oxygen) and at the ED (i.e. start of antibiotic treatment) and mortality (within 28 days) differed between non-documented and documented patients. The Dutch ambulance protocol for sepsis prescribes the administration of oxygen and intravenous fluids, if systolic blood pressure is below $90 \mathrm{mmHg}$ [22].

\section{Analysis}

All statistical analyses were processed with IBM SPSS Statistics 20.0. for Windows (IBM, Armont, New York, USA). Comparisons between variables were made using the Chi square Test or Fishers' exact test when appropriate. Means and medians were compared with the independent T-test and Mann-Whitney-U-test, respectively. Mortality rates were also presented in a Kaplan-Meier survival curve and differences between curves were calculated using the log rank test. P-values $<0.05$ were considered significant.

This study was approved by the Medical Ethics Committee of MUMC+. 


\section{Results}

\section{All sepsis patients}

In the study period, 7345 patients visited the internist at the ED. Of these, 867 (11.8\%) were diagnosed with sepsis at the ED (Figure 1). Of these patients, 101 (12.6\%) were excluded, mostly because of revisits $(n=53)$ or incomplete follow-up $(n=31)$. Of the remaining 766 (87.4\%) patients with sepsis, 363 patients $(47.4 \%)$ had been transported by ambulance.

Figure 1 Flowchart of the study population

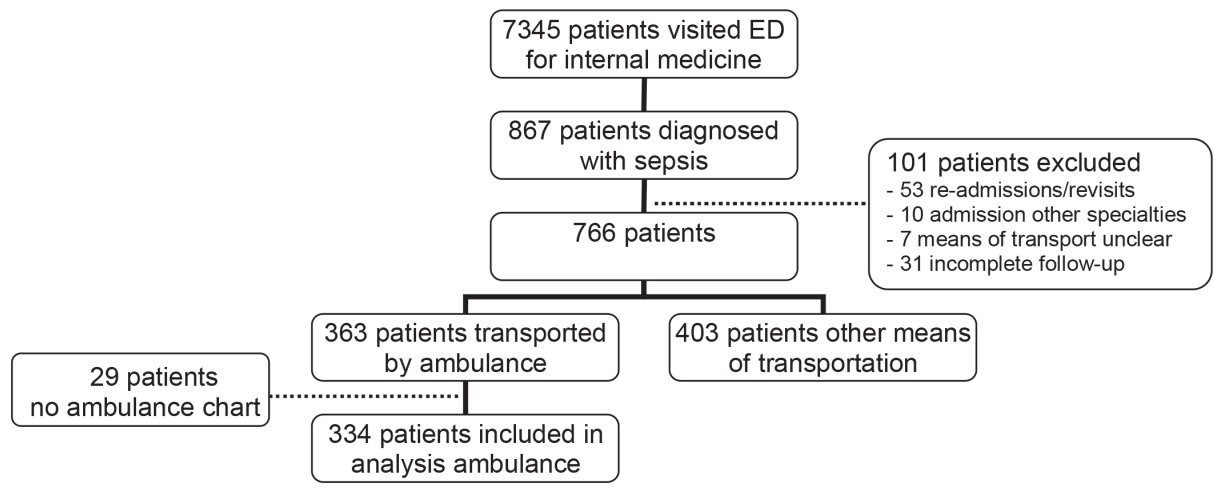

The ambulance patients were older (mean 71.5 vs. 55.7 years, $p<0.0001$ ) and more severely ill than those who were transported otherwise (Table 1 ). They more frequently had severe sepsis ( 47.4 vs. $25.8 \%, p<0.0001)$ or septic shock ( 12.4 vs. $4.0 \%, p<0.0001)$, had a higher MEWS at the ED (median 3 vs. 2, $p<0.0001$ ), were admitted more often to the hospital $(97.2$ vs. $85.4 \%, p<0.001)$ and the ICU (6.6 vs. $0.5 \%, p<0.001)$. The focus of infection in ambulance patients was more often respiratory than in patients transported otherwise ( 41.6 vs. $26.3 \%, p<0.001$ ). A1 and A2 journeys were equally time consuming ( $p=0.18)$. Ambulance patients stayed shorter at the ED than patients who were transported otherwise $(p=0.003)$. 
Table 1 Patient characteristics and outcome in all sepsis patients

\begin{tabular}{|c|c|c|}
\hline Number (\%) or median (IQR) & $\begin{array}{l}\text { Ambulance patients } \\
(n=363)\end{array}$ & $\begin{array}{l}\text { Patients transported } \\
\text { otherwise }(n=403)\end{array}$ \\
\hline Mean age $(S D)^{* \#}$ & $71.5( \pm 14.2)$ & $55.7( \pm 16.0)$ \\
\hline Male & $174(47.9)$ & $191(47.4)$ \\
\hline \multicolumn{3}{|l|}{ Co-morbidity } \\
\hline Malignancy* & $124(34.2)$ & $192(47.6)$ \\
\hline Cardiovascular disease* & $133(36.6)$ & $53(13.2)$ \\
\hline Diabetes* & $68(18.7)$ & 48 (11.9) \\
\hline Renal disease & $33(9.1)$ & $42(10.4)$ \\
\hline COPD* & $35(9.6)$ & $24(6.0)$ \\
\hline Cytotoxic medication* & $40(11.0)$ & 95 (23.6) \\
\hline Immune suppressive medication* & $81(22.3)$ & $172(42.7)$ \\
\hline \multicolumn{3}{|l|}{ Sepsis severity } \\
\hline Sepsis* & $146(40.2)$ & $283(70.2)$ \\
\hline Severe sepsis* & $172(47.4)$ & $104(25.8)$ \\
\hline Septic shock*\# & $45(12.4)$ & $16(4.0)$ \\
\hline MEWS* & $3(2-5)$ & $2(1-4)$ \\
\hline \multicolumn{3}{|l|}{ Focus of infection } \\
\hline Respiratory* & $146(40.2)$ & $106(26.3)$ \\
\hline Urinary & $88(24.2)$ & $76(18.9)$ \\
\hline Gastrointestinal & $34(9.4)$ & $60(14.9)$ \\
\hline Hepatobiliary & $18(5.0)$ & $30(7.4)$ \\
\hline Skin/joint & $21(5.8)$ & $33(8.2)$ \\
\hline Other/unknown* & $56(15.4)$ & $98(24.3)$ \\
\hline \multicolumn{3}{|l|}{ Outcome } \\
\hline Start of antibiotic treatment at the ED & $308(84.8)$ & $264(65.5)$ \\
\hline Length of ED stay (hours: minutes)*\# & 03:14 (02:37-04:01) & $03: 32(02: 47-04: 21)$ \\
\hline Admission to hospital* & $353(97.2)$ & $344(85.4)$ \\
\hline MCU-admission & $7(1.9)$ & $5(1.2)$ \\
\hline ICU-admission* & $24(6.6)$ & $2(0.5)$ \\
\hline Length of hospital stay (days)* & $7(4-13)$ & $5(3-11)$ \\
\hline Mortality within 28 days* & $65(17.9)$ & $29(7.2)$ \\
\hline
\end{tabular}


Mortality within 28 days in ambulance patients was higher than in those who were transported otherwise (17.9 vs. $7.2 \%, p<0.0001$, log rank test, Figure 2 ). Multivariate analysis showed that the factors that independently influenced mortality were age (HR 1.03 per year; $95 \% \mathrm{Cl} 1.01-1.04 ; \mathrm{p}=0.001$ ), septic shock (HR 3.1; 95\% Cl 1.6-6.0; $p=0.001$ ) and length of ED stay (HR 1.003; Cl 1.0004-1.01; $p=0.03$, Appendix, Table 2).

Figure 2 Kaplan Meier survival curve for ambulance patients and patients who were transported otherwise

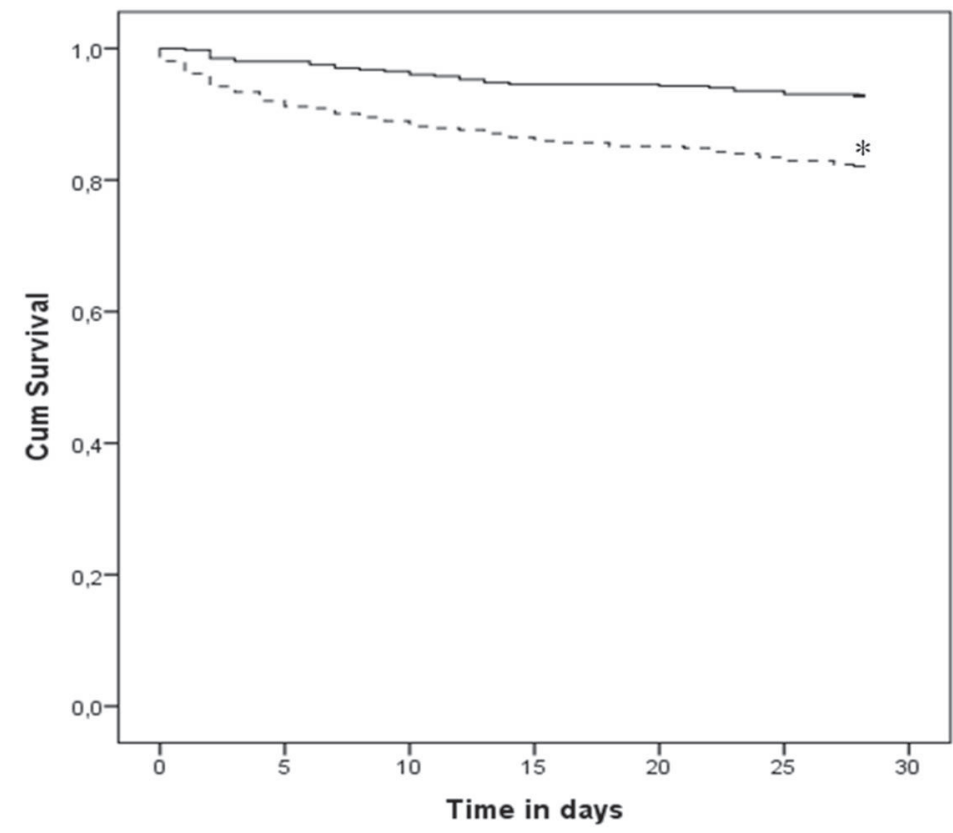

patients transported otherwise ambulance patients $p<0.0001$

\section{Ambulance patients with sepsis}

\section{Assessments during the ambulance journey}

For 334 (92.0\%) of the 363 patients who were transported by ambulance, the ambulance chart could be retrieved (Table 2). There were no differences in co-morbidity or use of cytotoxic or immune suppressive medication between the two groups. During the ambulance journey, respiratory rate was registered in $69.2 \%$, heart rate in $42.2 \%$ and temperature in $46.4 \%$ of the patients. All these 3 vital signs, the SIRS criteria, were registered in $20.4 \%$ of the patients. 
Table 2 Urgency, assessments, sepsis severity, treatment and mortality in non-documented and documented ambulance patients

\begin{tabular}{|c|c|c|c|}
\hline $\begin{array}{l}\text { Number (\%) or median } \\
\text { (IQR) }\end{array}$ & \begin{tabular}{|l|}
$\begin{array}{l}\text { Non-documented } \\
(\mathrm{N}=140)\end{array}$ \\
\end{tabular} & $\begin{array}{l}\text { Documented } \\
(\mathrm{N}=194)\end{array}$ & $\begin{array}{l}\text { All } \\
(\mathrm{N}=334)\end{array}$ \\
\hline Age (mean, SD) & $72.5( \pm 13.5)$ & $72.2( \pm 14.0)$ & $72.3( \pm 13.8)$ \\
\hline \multicolumn{4}{|l|}{ Co-morbidity } \\
\hline Malignancy & $41(29.3)$ & $66(34.0)$ & $107(32.0)$ \\
\hline Cardiovascular disease & $51(36.4)$ & $74(38.1)$ & $125(36.4)$ \\
\hline Diabetes & $28(20)$ & $37(19.1)$ & 65 (19) \\
\hline Renal disease & $11(7.9)$ & $20(10.3)$ & $31(9.3)$ \\
\hline COPD & $13(9.3)$ & $17(8.8)$ & $30(8.7)$ \\
\hline Cytotoxic medication & $15(10.7)$ & $21(10.8)$ & $36(10.8)$ \\
\hline $\begin{array}{l}\text { Immune suppressive } \\
\text { medication }\end{array}$ & $27(19.3)$ & $41(21.1)$ & $68(20.4)$ \\
\hline $\begin{array}{l}\text { Referral by general } \\
\text { practitioner }\end{array}$ & $74(52.9)$ & $117(60.3)$ & $191(57.2)$ \\
\hline \multicolumn{4}{|l|}{ Ambulance urgency*\# } \\
\hline Non urgent (B-journey) & $58(41.4)$ & $108(55.7)$ & $166(49.7)$ \\
\hline $\begin{array}{l}\text { Urgent (A1- and A2- } \\
\text { journey) }\end{array}$ & $82(58.6)$ & $86(44.3)$ & $168(50.3)$ \\
\hline \multicolumn{4}{|c|}{ Ambulance assessments of vital signs } \\
\hline Respiratory rate & $100(71.4)$ & $131(67.5)$ & $231(69.2)$ \\
\hline Heart rate & $58(41.4)$ & $83(42.8)$ & $141(42.2)$ \\
\hline Blood pressure & $101(72.1)$ & $125(64.4)$ & $226(67.7)$ \\
\hline Mental state & $87(62.1)$ & $102(52.6)$ & $189(56.7)$ \\
\hline Body temperature*\# & $23(16.4)$ & $132(68.0)$ & $155(46.4)$ \\
\hline Oxygen saturation & $64(45.7)$ & $93(47.9)$ & $157(47.0)$ \\
\hline \multicolumn{4}{|c|}{ SIRS assessments in ambulance } \\
\hline None* & $35(25.0)$ & $16(8.2)$ & $51(15.3)$ \\
\hline 1 of 3 & $42(30.0)$ & $65(33.5)$ & $107(32.0)$ \\
\hline 2 of 3 & $50(35.7)$ & $58(29.9)$ & $108(32.3)$ \\
\hline All 3* & $13(9.3)$ & $55(28.4)$ & $68(20.4)$ \\
\hline \multicolumn{4}{|l|}{ Sepsis severity } \\
\hline Sepsis & $47(33.6)$ & $80(41.2)$ & $127(38.0)$ \\
\hline Severe sepsis & $71(50.7)$ & $94(48.5)$ & $165(49.3)$ \\
\hline Septic shock & $22(15.7)$ & $20(10.3)$ & $42(12.6)$ \\
\hline MEWS in ambulance* & $1.5(0-3)$ & $2(2-4)$ & $2(0-4)$ \\
\hline MEWS at the ED & $3(2-5)$ & $3.5(2-5)$ & $3(2-5)$ \\
\hline
\end{tabular}




\begin{tabular}{|c|c|c|c|}
\hline $\begin{array}{l}\text { Number (\%) or median } \\
\text { (IQR) }\end{array}$ & \begin{tabular}{|l|} 
Non-documented \\
$(\mathrm{N}=140)$
\end{tabular} & \begin{tabular}{|l} 
Documented \\
( $N=194)$
\end{tabular} & $\begin{array}{l}\text { All } \\
(\mathrm{N}=334)\end{array}$ \\
\hline Age (mean, SD) & $72.5( \pm 13.5)$ & $72.2( \pm 14.0)$ & $72.3( \pm 13.8)$ \\
\hline \multicolumn{4}{|l|}{ Focus of infection } \\
\hline Respiratory & $52(37.1)$ & $84(43.3)$ & $136(40.7)$ \\
\hline Urinary & $31(22.1)$ & $50(25.8)$ & $81(24.3)$ \\
\hline Gastrointestinal & $17(12.1)$ & $10(5.2)$ & $27(8.1)$ \\
\hline Hepatobiliary & $10(7.1)$ & $6(3.1)$ & $16(4.8)$ \\
\hline Skin/joint* & $3(2.1)$ & $15(7.7)$ & $18(5.4)$ \\
\hline Other/unknown & $27(19.3)$ & $29(14.9)$ & $56(16.8)$ \\
\hline \multicolumn{4}{|l|}{ Outcomes } \\
\hline Ambulance treatment & $116(82.3)$ & $170(87.6)$ & $286(85.6)$ \\
\hline Intravenous fluid & 39 (27.9) & $51(26.3)$ & $90(26.9)$ \\
\hline Oxygen & $59(42.1)$ & $68(35.1)$ & $127(38.0)$ \\
\hline ED stay (hours: minutes)* & $\begin{array}{r}03: 34 \\
(02.47-04: 26) \\
\end{array}$ & $\begin{array}{r}03: 04 \\
(02: 37-03: 50) \\
\end{array}$ & $\begin{array}{r}03: 14 \\
(02: 38-04: 04)\end{array}$ \\
\hline $\begin{array}{l}\text { Start of antibiotic } \\
\text { treatment at the ED }\end{array}$ & $116(82.3)$ & $170(87.6)$ & $286(85.6)$ \\
\hline ED administration IV fluid & $98(70.0)$ & $129(66.5)$ & $227(68.0)$ \\
\hline Mortality within 28 days* & $36(25.7)$ & $25(12.9)$ & $61(18.3)$ \\
\hline \multicolumn{4}{|c|}{$\begin{array}{l}\mathrm{SD}=\text { standard deviation; } \mathrm{IQR}=\text { inter quartile range. } \\
* \mathrm{p}<0.05 \text { between the documented and non-documented patients; \# independent factors of importance } \\
\text { for documentation of sepsis. }\end{array}$} \\
\hline
\end{tabular}

\section{Documentation during the ambulance journey}

In 140 (41.9\%) patients, the diagnosis sepsis was not documented by ambulance staff. These non-documented patients were transported more frequently with an urgent journey (Table 2, $\mathrm{p}=0.01$ ) than documented patients. Non-documented patients had a lower MEWS score in the ambulance than documented patients (median 1.5 vs. $2, p<0.0001$ ). Body temperature ( 16.4 vs. $68.0 \%, p<0.0001)$ and all 3 SIRS criteria $(9.3$ vs. $28.4 \%, p<0.0001)$ were measured less often in non-documented patients.

Two factors were independently associated with documentation of sepsis: first, the urgency of the journey (urgent transport had a negative association with documentation (OR 0.5; 95\% $\mathrm{Cl} 0.3-0.9 ; \mathrm{p}=0.02$ )) and second, assessment of body temperature (positive association with documentation (OR 11.2; 95\% Cl 5.2-24.4; p<0.0001, Appendix, Table 3).

In 65 (46.4\%) non-documented patients, the laboratory values "leukocytes" or "pCO2" were needed as second SIRS criterion to diagnose this sepsis (Table 3). In $32.1 \%$, the vital signs needed to identify sepsis were not assessed and $15.7 \%$ of the patients were not documented as septic, despite the presence of 2 SIRS criteria. 
Table 3 Presence and assessment of SIRS criteria during the ambulance journey in non-documented patients

\begin{tabular}{|l|r|}
\hline & Number (\%) \\
\hline Prehospital vital signs did not meet sepsis criteria & $8(5.7)$ \\
\hline Vital signs needed to identify sepsis not assessed in ambulance & $45(32.1)$ \\
\hline Patients identified at the ED by leukocyte count or low pCO2 & $65(46.4)$ \\
\hline Sepsis not documented despite presence of 2 SIRS criteria & $22(15.7)$ \\
\hline
\end{tabular}

Treatment and outcome of ambulance patients

Non-documented patients were not treated differently (in both ambulance and ED) than documented patients regarding administration of fluid and oxygen (Table 2). There was no difference in the number of non-documented and documented patients, who received antibiotics ( 82.3 vs.87.6\%, $p=0.22$ ). The length of stay at the ED was longer in non-documented patients (median time 03:34 vs. 03:04, $p=0.01$ ). Mortality within 28 days was higher in non-documented patients ( 25.7 vs. $12.9 \%, p=0.002$, log rank test, Figure 3 ).

Figure 3 Kaplan Meier survival curve in ambulance patients

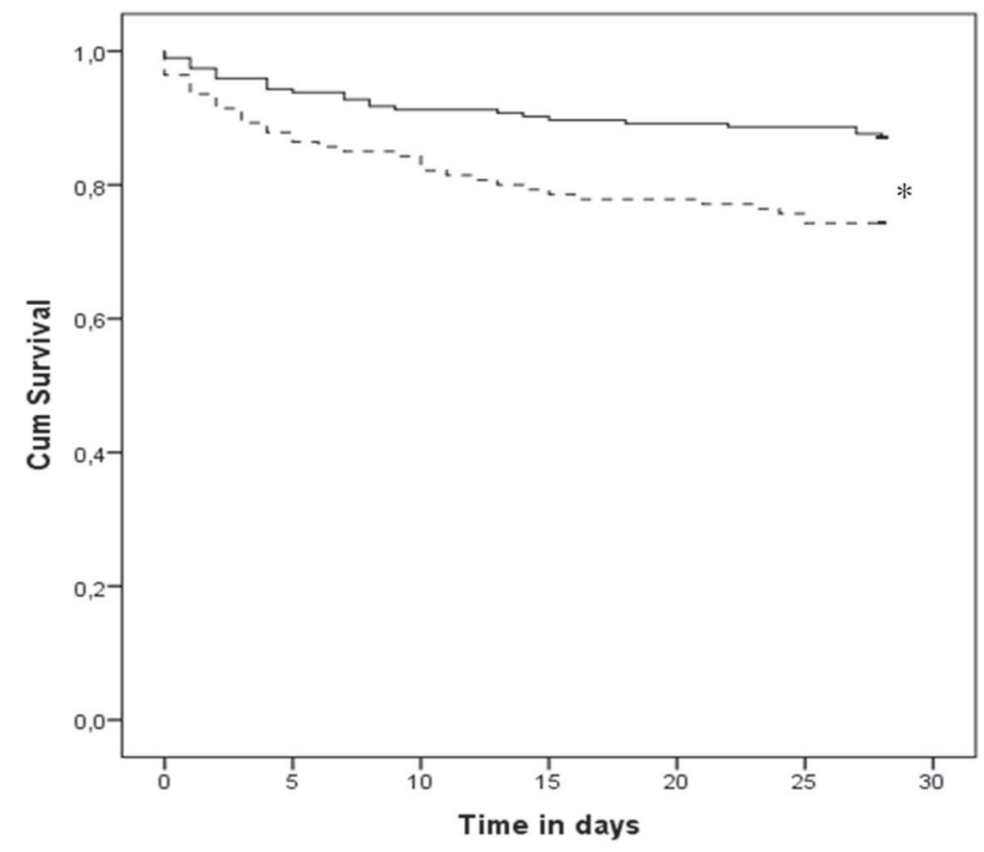

documented patients

- - - non-documented patients

$p=0.002$ 


\section{Discussion}

Almost half (47.4\%) of the patients with sepsis, who visit the internist at the ED, are transported by ambulance. These ambulance patients are older, more severely ill, admitted to the hospital more frequently and have a higher 28-day mortality rate than those who are transported otherwise. Unfortunately, $41.9 \%$ of the ambulance patients are not documented to be septic by ambulance staff.

Our finding that $47.4 \%$ of septic patients are transported by ambulance is in agreement with other studies[9, 23]. It is important to realize that we studied medical patients only and therefore, we cannot extrapolate to the total number of ambulance patients with sepsis. We found that the ambulance transports the most severely ill sepsis patients, which is in agreement with other studies[9, 23]. Ambulance patients in our study died more frequently (17.9\%) than those who were transported otherwise $(7.2 \%$, $\mathrm{p}<0.0001$ ). This difference can (in part) be explained by the higher age of the ambulance patients (HR 1.03 per year) but also by higher sepsis severity (HR 3.1 for septic shock). The severity of illness of ambulance patients indicates that ambulance services may play an important role in the improvement of care for sepsis patients, because these severely ill patients are known to benefit from early start of treatment $[12,23]$.

In order to improve the care for septic patients in the ambulance by early initiation of treatment in the ambulance, they first need to be recognized as having sepsis. Since this is a retrospective study, we cannot conclude on the exact number of patients in whom the diagnosis sepsis was recognized, as we were only able to retrieve the cases of sepsis that were documented (58.1\%). Urgent transport was negatively associated with documentation. This might be explained by the fact that the ambulance nurse needs time to treat the patient and has less time to document sepsis in the ambulance chart. However, treatment between the documented and non-documented patients was not different.

An accurate assessment of vital signs and thus the SIRS criteria may improve sepsis recognition and thereby possibly improve treatment and outcome. We found this to be true particularly for temperature, as this vital sign contributed most to the documentation of sepsis (OR 11.2). However, measuring the temperature in the ambulance is not common in all countries. Our finding suggests that measuring the temperature in the ambulance can help to improve early recognition of sepsis. In accordance, not assessing the vital signs/SIRS criteria explained $32.1 \%$ of the non-documented cases. Part of the non-assessment could be explained by the fact that for a severely ill patient, the priority of the nurse shifts from assessing the patient to immediate transport to the hospital, also known as 'scoop and run'. However, assessing the SIRS criteria can never result in $100 \%$ recognition of sepsis in the ambulance, as not all SIRS criteria (the laboratory values leukocytes, leukocyte bands and pCO2) can be measured in the ambulance (which explained $46.4 \%$ of the non-documented cases), in $5.7 \%$ sepsis was not present in the ambulance and in $15.7 \%$ the diagnosis was missed despite presence of 2 SIRS criteria. This is in accordance with an American study that found that $60 \%$ of missed sepsis could 
be explained by missing laboratory values, $14.3 \%$ of patients did not meet sepsis criteria in the ambulance and $25.7 \%$ was not recognized despite presence of sepsis criteria[14]. In conclusion, in $47.8 \%$ of the non-documented patients, sepsis can be recognized when all vital signs are assessed and knowledge on sepsis is improved.

Early recognition of sepsis is important, because we, like others, found that non-documented patients had a higher mortality than documented patients ( 25.7 vs. $12.9 \%$, $p<0.001)[14]$. We could not explain this difference in mortality by differences in age, comorbidity, the use of cytotoxic or immune suppressive medication, sepsis severity, initiated treatment in the ambulance and start of antibiotic treatment at the ED. The MEWS in the ambulance was lower in non-documented patients than in documented patients (1.5 vs. 2). This difference can be explained by the fact that in non-documented patients, less assessments were performed in the ambulance. In addition, the MEWS at the ED was equal in both groups. Non-documented patients stayed half an hour longer at the $E D$, which may indicate that treatment at the ED was started later. In this study we were not able to retrieve the data to prove this hypothesis. However, in other studies, patients who were recognized as septic by the paramedic received antibiotics 53 minutes earlier at the ED than those who were not recognized[9].

Overall, treatment of sepsis is presently not often initiated during the ambulance ride. Intravenous fluid was administered in $26.9 \%$, which is consistent with other studies, wherein pre-hospital fluid was administered in $30-48 \%$ of patients with severe sepsis[10-12]. These findings can at least in part be explained by the current ambulance protocol, which only prescribes oxygen and intravenous fluids in case of shock [22]. In this study, we were not able to quantify the treatment that was given. It is therefore possible that documented patients received a larger amount of fluid and oxygen than the non-documented patients. In other studies, patients who were treated with intravenous fluids in the ambulance (irrespective of the presence of shock), received twice the volume of fluids in the first hour of ED stay and had a better outcome (less intubations and lower mortality) $[12,23]$.

\section{Limitations}

We included sepsis patients according to the classical sepsis definition of Bone, et al. [18], like others did [24, 25]. Recently, there is growing concern about the sensitivity and specificity of these criteria [17]. Unfortunately, despite efforts to improve the definition of sepsis, there is no extensively tested and superior definition of sepsis which can be used in research. Further, we can only rely on registered values. It is therefore possible that vital signs were assessed more frequently than we retrieved in the charts. This will not be an issue in future research as the assessments of vital signs will be registered automatically in our ambulances in the near future.

Our ambulance team consists of a trained ambulance driver and a highly trained nurse, which is different to the situation in some other countries. However, our findings concerning the recognition of sepsis are also in accordance with an American study [14]. 


\section{Future perspectives}

Further research in measuring vital signs, recognition, documentation, treatment and outcome of sepsis in the ambulance is needed. A prospective study can provide more precise data on sepsis recognition by ambulance staff and maybe, the whole emergency care chain. However, it would be a challenge to design this study in such a way that the study does not influence (i.e. improve) recognition by increasing the awareness for sepsis.

Our study suggests that training of ambulance staff, and maybe employees of the dispatch centre and general practitioners, could improve sepsis recognition.

\section{Conclusion}

Almost half (47.4\%) of all sepsis patients, who visit the internist at the ED, are transported by ambulance. These patients are older, more severely ill and have a higher 28-day mortality rate than patients transported otherwise.

In $41.9 \%$ of the ambulance patients, sepsis is not documented in the ambulance chart and in this group, 28-day mortality is twice as high as in patients with documented sepsis. Not all sepsis patients can be identified in the ambulance, mostly because laboratory values cannot be measured. However, $47.8 \%$ of the non-documented patients could probably have been identified by assessing the vital signs, or at least a temperature, and by increasing the knowledge on sepsis.

\section{Acknowledgements}

H. Coenen, head of the emergency medical dispatch centre RAV-ZL,

N. Otten, ICT specialist emergency medical dispatch centre RAV-ZL,

G. Vandergraesen, staff supporter of the ED MUMC+ 


\section{References}

1. Gaieski DF, Edwards JM, Kallan MJ, Carr BG. Benchmarking the Incidence and Mortality of Severe Sepsis in the United States. Crit Care Med 2013;5:1167-74.

2. Martin CM, Priestap F, Fisher H, Fowler RA, Heyland DK, Keenan SP, et al. A prospective, observational registry of patients with severe sepsis: the Canadian Sepsis Treatment and Response Registry. Crit Care Med 2009;1:81-8.

3. Kumar A, Roberts D, Wood KE, Light B, Parrillo JE, Sharma S, et al. Duration of hypotension before initiation of effective antimicrobial therapy is the critical determinant of survival in human septic shock. Crit Care Med 2006;6:1589-96.

4. Rivers E, Nguyen B, Havstad S, Ressler J, Muzzin A, Knoblich B, et al. Early goal-directed therapy in the treatment of severe sepsis and septic shock. New Engl J Med 2001;19:1368-77.

5. Dellinger RP, Carlet JM, Masur H, Gerlach H, Calandra T, Cohen J, et al. Surviving Sepsis Campaign guidelines for management of severe sepsis and septic shock. Crit Care Med 2004;3:858-73.

6. Shapiro NI, Howell MD, Talmor D, Lahey D, Ngo L, Buras J, et al. Implementation and outcomes of the Multiple Urgent Sepsis Therapies (MUST) protocol. Crit Care Med 2006;4:1025-32.

7. Nguyen HB, Corbett SW, Steele R, Banta J, Clark RT, Hayes SR, et al. Implementation of a bundle of quality indicators for the early management of severe sepsis and septic shock is associated with decreased mortality. Crit Care Med 2007;4:1105-12.

8. Su F, Nguyen ND, Creteur J, Cai Y, Nagy N, Anh-Dung H, et al. Use of low tidal volume in septic shock may decrease severity of subsequent acute lung injury. Shock 2004;2:145-50.

9. Studnek JR, Artho MR, Garner CL, Jr., Jones AE. The impact of emergency medical services on the ED care of severe sepsis. Am J Emerg Med 2012;1:51-6.

10. Seymour CW, Rea TD, Kahn JM, Walkey AJ, Yealy DM, Angus DC. Severe sepsis in pre-hospital emergency care: analysis of incidence, care, and outcome. Am J Resp Crit Care Med 2012;12:1264-71.

11. Seymour CW, Band RA, Cooke CR, Mikkelsen ME, Hylton J, Rea TD, et al. Out-of-hospital characteristics and care of patients with severe sepsis: a cohort study. J Crit Care 2010;4:553-62.

12. Seymour CW, Cooke CR, Mikkelsen ME, Hylton J, Rea TD, Goss CH, et al. Out-of-hospital fluid in severe sepsis: effect on early resuscitation in the emergency department. Prehos Emerg Care 2010;2:145-52.

13. Seymour CW, Cooke CR, Heckbert SR, Spertus JA, Callaway CW, Martin-Gill C, et al. Prehospital intravenous access and fluid resuscitation in severe sepsis: an observational cohort study. Crit Care 2014;5:533.

14. Guerra WF, Mayfield TR, Meyers MS, Clouatre AE, Riccio JC. Early detection and treatment of patients with severe sepsis by prehospital personnel. J Emerg Med 2013;6:1116-25.

15. Thijssen WA, Giezen PH, Wensing M. Emergency departments in The Netherlands. Emerg Med J 2012;1:6-9

16. Groenewoudt M, Roest AA, Leijten FM, Stassen PM. Septic patients arriving with emergency medical services: a seriously ill population. Eur J Emerg Med 2014;5: 330-5.

17. Levy MM, Fink MP, Marshall JC, Abraham E, Angus D, Cook D, et al. 2001 SCCM/ESICM/ACCP/ATS/SIS International Sepsis Definitions Conference. Crit Care Med 2003;4:1250-6.

18. Bone RC, Balk RA, Cerra FB, Dellinger RP, Fein AM, Knaus WA, et al. Definitions for sepsis and organ failure and guidelines for the use of innovative therapies in sepsis. The ACCP/SCCM Consensus Conference Committee. Chest 1992;6:1644-55.

19. Subbe CP, Kruger M, Rutherford P, Gemmell L. Validation of a modified Early Warning Score in medical admissions. QJM 2001;10:521-6.

20. Geier F, Popp S, Greve Y, Achterberg A, Glöckner E, Ziegler R, et al. Severity illness scoring systems for early identification and prediction of in-hospital mortality in patients with suspected sepsis presenting to the emergency department. Wien Klin Wochenschr 2013;17-18:508-15.

21. Cildir E, Bulut M, Akalin H, Kocabaş E, Ocakoğlu G, Aydın ŞA. Evaluation of the modified MEDS, MEWS score and Charlson comorbidity index in patients with community acquired sepsis in the emergency department. Intern Emerg Med 2013;3:255-60.

22. Anonymous. [National protocol ambulance service, version 7.2] Landelijk protocol ambulancezorg versie 7.22011 . 
Chapter 4

23. Band RA, Gaieski DF, Hylton JH, Shofer FS, Goyal M, Meisel ZF. Arriving by emergency medical services improves time to treatment endpoints for patients with severe sepsis or septic shock. Acad Emerg Med 201;9:934-40.

24. Pro $\mathrm{Cl}$, Yealy DM, Kellum JA, Huang DT, Barnato AE, Weissfeld LA, et al. A randomized trial of protocolbased care for early septic shock. New Engl J Med 2014;18:1683-93.

25. Investigators A, Group ACT, Peake SL, Delaney A, Bailey M, Bellomo R, et al. Goal-directed resuscitation for patients with early septic shock. New Engl J Med 2014;16:1496-506. 


\section{Section III}

Risk stratification of sepsis patients at the Emergency Department 



\section{Chapter}

Predictive Accuracy and Feasibility of
Risk Stratification Scores for 28-day
Mortality of Patients with Sepsis in
an Emergency Department

Michelle J.M. Hilderink*, Asselina A. Roest*, Maud Hermans, Yolande C. Keulemans, Coen D.A. Stehouwer, Patricia M. Stassen $(*$ both authors contributed equally)

European Journal of Emergency Medicine 2015;22(5):331-7 


\begin{abstract}

\section{Objectives}

Sepsis is associated with high mortality. Because early therapy has proven to decrease mortality, a risk stratification tool that quickly and easily quantifies mortality risk of patients will be helpful to guide appropriate treatment. We investigated 5 scores regarding a) predicting 28-day mortality and b) their feasibility for use in the emergency department (ED)

\section{Methods}

We carried out a historical cohort study at the ED of Maastricht University Medical Centre. Patients who fulfilled criteria for sepsis were included if they had been admitted to the hospital by an internist between August 2009 and August 2010. The Mortality in Emergency Department Sepsis (MEDS), Confusion, Urea, Respiratory rate, Blood pressure, age > 65 (CURB-65), Acute Physiology And Chronic Health Evaluation II (APACHE II), Rapid Acute Physiology Score (RAPS) and Rapid Emergency Medicine Score (REMS) scores were calculated using ED charts. The primary outcome was total 28-day mortality. Receiver operating characteristics (ROC) curves and calibration plots were constructed to evaluate predictive accuracy. Feasibility was defined as the proportion of patients for whom all data were available.
\end{abstract}

\title{
Results
}

We included 600 patients, of whom 90 (15\%) died within 28 days. Discriminating ability for total 28-day mortality of the MEDS (area under the curve (AUC): $0.82,95 \%$ confidence interval (CI) 0.78-0.87), CURB-65 (AUC: $0.78,95 \% \mathrm{Cl} 0.73-0.83$ ) and APACHE II (AUC: 0.71, $95 \% \mathrm{Cl}$ 0.64-0.79) were highest, but only the difference between the MEDS and REMS ( $\mathrm{P}=$ $0.007)$ and RAPS score $(P<0.001)$ was significant. Both the MEDS and CURB-65 had higher AUCs for predicting 28-day in-hospital mortality than the other 3 scores, but this was only significant for the MEDS score compared with the RAPS $(P=0.003)$. Both the MEDS and CURB-65 underestimated mortality, especially for the higher scores. The MEDS, CURB-65, REMS and RAPS were most feasible as they could be calculated in more than $96 \%$ of patients.

\section{Conclusion}

The MEDS and CURB-65 scores are the most adequate and feasible tools for the prediction of total 28-day mortality in septic patients presenting at the ED, but they need local recalibration before use at the ED. 


\title{
Introduction
}

\author{
Background
}

Sepsis is a systemic response to infection [1]. It represents a major health care burden and is often lethal with a mortality that is equal to that of myocardial infarction or stroke [2]. On the basis of their clinical presentation, patients who fulfil the criteria of systemic inflammatory response syndrome (SIRS) and are suspected to have an infection, can be classified into 3 severity categories of the sepsis syndrome: sepsis, severe sepsis and septic shock [1]. The in-hospital mortality among patients increases with disease severity from $16 \%$ for patients with sepsis, $20 \%$ for patients with severe sepsis, and $40 \%$ for patients with septic shock [3]. Despite these high mortality rates, the sepsis syndrome is regularly overlooked and its significance is frequently underestimated by emergency department (ED) staff [4].

\section{Importance}

One of the most significant recent improvements in sepsis treatment was the introduction of early goal-directed therapy (EGDT), which is a multifaceted strategy to rapidly correct the physiological derangements in sepsis patients [5]. Implementation of EGDT has proved to reduce mortality and sepsis-related costs [5-9]. Therefore, an easy and accurate scoring system that enables quickly quantification of a patient's risk of dying may be helpful to make decisions on treatment by increasing the treating physician's awareness of the risks involved.

Several scoring systems have been developed to predict mortality in patients with sepsis syndrome or sepsis related organ failure. We evaluated and compared 5 scoring systems: the 'Mortality in Emergency Department Sepsis' (MEDS), 'Confusion, Urea, Respiratory rate, Blood pressure, age $>65$ ' (CURB-65), 'Acute Physiology And Chronic Health Evaluation II' (APACHE II), 'Rapid Acute Physiology Score' (RAPS) and 'Rapid Emergency Medicine Score' (REMS) [10-14]. The MEDS, CURB-65 and REMS were specifically designed for use in an ED setting or on admission to the hospital, whereas the other scoring systems were developed in other settings but have also been applied in EDs [15-18]. The MEDS, APACHE II and RAPS were developed in the United States; the CURB was developed in the United Kingdom and the REMS in Sweden. The 5 scoring systems have not been compared in the same ED patient population before.

\section{Goals of this investigation}

To assess the generalizability of these scoring systems, it is important to study how well scoring systems perform in other countries than where they have been developed. Therefore, we assessed, and mutually compared, the prognostic accuracy and feasibility of these scoring systems in patients with the sepsis syndrome at the ED of a Dutch hospital. 


\title{
Materials and methods
}

\author{
Study design, setting and selection of participants
}

We carried out a study in a historical cohort in the Maastricht University Medical Centre (MUMC) which is a university hospital in the Netherlands that provides secondary and tertiary healthcare. On average, the ED of the hospital is annually visited by 22,750 patients of whom 5,075 are specifically referred to an internist. Apart from high emergency (ambulance) patients and a few self-presenters, in the Netherlands most patients visit the ED after referral by a general practitioner. In our ED, internists specialized in acute medicine and their residents assess all such patients, and decide on further treatment including admittance to the hospital and consultation of an intensivist. In our hospital, there are no emergency physicians and risk stratification tools are not used for the care of sepsis patients at the ED.

We reviewed the ED charts of every patient who attended the ED between 1 August 2009 and 31 July 2010 and was examined by an internist. Consecutive patients, who visited the ED for the first time within this period, were 18 years or older, fulfilled the clinical criteria for sepsis, severe sepsis or septic shock, irrespective of the probable origin of the sepsis, and who had been admitted to the hospital, were included in the study. The diagnosis of sepsis and its severity were established at the ED using data from the electronic ED and hospital chart by one investigator, which was confirmed by a second. In case of doubt, a third investigator decided on this issue. Standardized forms were used to extract the data from the ED and hospital charts. Visits of patients who were admitted more than once in the study period, were excluded, except for the first admittance. The study was approved by the local Medical Ethics Committee.

\section{Scoring systems}

We selected scoring systems that could be used in adult patients at the ED. We excluded scoring systems that require items that are not available in our ED, such as urine output and central venous catheter measurements. Furthermore, as blood gas analysis is not performed on a routine basis in our patients, we included only one score, that requires this analysis, the APACHE II.

The 5 scoring systems are summarized in Table 1 . The MEDS score was developed for ED patients with suspected infection [10]. Patients are divided into 5 severity categories to predict 28-day in-hospital mortality. The CURB-65 score was developed to assess disease severity in patients with community-acquired pneumonia and to predict 30-day mortality [11]. The score is not considered applicable when suspicion of pneumonia is not the primary reason for hospital admission, when patients are immunocompromised, live in nursing homes, have underlying lung disease or haematological malignancies. Because many patients with suspected infection should officially be excluded, we evaluated the predictive accuracy of the CURB-65 for our whole cohort and also for the subgroup of patients on the basis of the original selection criteria [11]. The APACHE II score originally aimed to assess disease severity in adults during the first 24 hours of admission to an ICU [12]. Higher scores are associated with higher mortality. The RAPS was developed as an abbreviated version of the APACHE II and predicts mortality 24 
hours after transfer of critically ill patients between hospitals [13]. The REMS is an extended version of the RAPS and was designed to predict mortality in nonsurgical ED patients [14].

Table 1 Selected scoring systems

\begin{tabular}{|c|c|c|c|c|c|}
\hline Scores & MEDS (10) & CURB-65 (11) & APACHE II(12) & RAPS (13) & REMS (14) \\
\hline Items & \begin{tabular}{|l} 
- tachypnea or \\
hypoxia \\
- shock \\
- altered \\
mental state \\
- age \\
- bands \\
- thrombocytes \\
- lower \\
respiratory \\
infection \\
- nursing home \\
resident \\
- terminal \\
illness
\end{tabular} & $\begin{array}{l}\text { - respiratory } \\
\text { rate } \\
\text { - low systolic } \\
\text { or diastolic } \\
\text { BP } \\
\text { - confusion } \\
\text { - age } \\
\text { - urea }\end{array}$ & \begin{tabular}{|l|} 
- respiratory \\
- rate \\
- GCart rate \\
- age \\
- creatinine \\
- temperature \\
- MAP \\
- haematocrit \\
- arterial pH \\
- chronic organ \\
insufficiency or \\
immunocom- \\
promised \\
- sodium \\
- potassium \\
- leukocytes \\
- (A-a)PO2 or \\
PAO2
\end{tabular} & $\begin{array}{l}\text { - } \text { respiratory } \\
\text { rate } \\
\text { - } \mathrm{MAP} \\
\text { - } \mathrm{GCS} \\
\text { - } \text { heart rate }\end{array}$ & $\begin{array}{l}\text { - respiratory } \\
\text { rate } \\
\text { - MAP } \\
\text { - GCS } \\
\text { - heart rate } \\
\text { - age } \\
\text { - temperature } \\
\text { - peripheral } \\
\text { oxygen } \\
\text { saturation }\end{array}$ \\
\hline $\begin{array}{l}\text { Intended } \\
\text { endpoint }\end{array}$ & $\begin{array}{l}\text { mortality in } \\
\text { septic patients } \\
\text { at the ED }\end{array}$ & \begin{tabular}{|l|} 
mortality in \\
patients with \\
community \\
acquired \\
pneumonia \\
\end{tabular} & $\begin{array}{l}\text { mortality } \\
\text { in patients } \\
\text { admitted to the } \\
\text { ICU }\end{array}$ & $\begin{array}{l}\text { mortality } \\
\text { in patients } \\
\text { being } \\
\text { transported }\end{array}$ & $\begin{array}{l}\text { mortality in } \\
\text { patients in } \\
\text { non-surgical } \\
\text { ED }\end{array}$ \\
\hline Mortality & $\begin{array}{l}28 \text { days } \\
\text { in-hospital }\end{array}$ & 30 days & 24 hours & 24 hours & 28 days \\
\hline Range & $0-27$ & $0-5$ & $0-71$ & $0-16$ & $0-30$ \\
\hline
\end{tabular}

\section{Data collection and processing}

All data were retrieved retrospectively from the ED charts and the electronic hospital database: demographic data, vital parameters, severity of sepsis, working diagnosis, focus of infection, comorbidity, chemotherapy within the past 28 days, recent immunosuppressive therapy and laboratory results. The data used for analysis were those present at the ED visit. Because the Glasgow Coma Scale (GCS) and respiratory rate were not always recorded exactly at the ED charts, these items were scored by deduction from other items in the chart. For GCS values that were not noted exactly, the description "alert" was considered "15" and a change in mental state was scored as "14", like others did [16]. Similarly, for 
respiratory rates, we converted "normal respiratory rate" to $14 /$ minute, as this value is considered normal in all 5 scores, and "tachypnoic" to 30/minute, as this is considered abnormally high in all 5 scores as well. We, like others, organized periodic meetings throughout the progress of our study to increase the quality of our deductions [19].

\section{Outcome measures}

The primary endpoint in our study was all-cause mortality within 28 days, either in-hospital or after discharge. Although the choice of in-hospital mortality as second endpoint is arbitrary, it was chosen because it was also used to develop and validate the MEDS in earlier studies, and therefore results can be better compared $[10,16]$. We retrieved data on mortality by checking the electronic hospital database or, if necessary, by contacting the patient's general practitioner.

\section{Data analysis}

We assessed two aspects of accuracy of the scoring systems to predict mortality: discrimination and calibration. To quantify discriminating ability, receiver operating characteristics (ROC) curves were constructed, and area under the curves (AUCS) with their 95\% confidence interval $(\mathrm{Cl})$ were calculated. Statistical testing for differences between AUCs was performed using the DeLong test [20].

We assessed the generalizability of risk estimates (calibration) of the scoring systems by comparing mortality rates in our population (observed mortality) with those reported in the original validation studies (expected mortality) in calibration plots. Expected mortality estimates could only be retrieved for the MEDS, CURB-65 and APACHE II scores [10-12].

The feasibility of the scoring systems was expressed as the proportion of patients for whom the scoring systems could be calculated. To investigate why the scoring systems could not be calculated in some of the patients, we assessed the frequency of incomplete scores is subgroups based on patient characteristics, complaints at presentation and comorbidity.

All statistical analyses were performed with SPSS for Windows 20.0 (IBM, Armont, New York, USA, 2011) and MedCalc for Windows, version 12.4.0.0 (MedCalc Software, Mariakerke, Belgium). Categorical data were summarized as percentages, and continuous data were reported as means with standard deviations (SD). Differences were considered statistically significant if P-value was less than 0.05 .

\section{Results}

In the 12-month study period, 5053 patients were assessed by an internist at the ED, of whom 2150 were admitted to the hospital. Of these patients, 664 (30.9\%) were suspected of having sepsis, severe sepsis or septic shock at the moment of presentation at the ED (Table 2 ). Four ( $0.6 \%$ ) of these were excluded because data on essential selection criteria like blood pressure were missing and 60 because it was not their first visit within the study period. Therefore, 600 patients were analyzed. 
Sepsis was the most prevalent category of the sepsis syndrome (57.3\%), followed by severe sepsis (36.7\%) and septic shock (6.0\%) (Table 2). Of the 600 patients, 75 patients (12.5\%) died in the hospital within 28 days, while another 15 (2.5\%) also died within 28 days, but after discharge. Total 28 -day mortality was $15.0 \%$.

Table 2 Patient characteristics and management

\begin{tabular}{|c|c|}
\hline All patients $\mathrm{n}(\%)$ & $n=600$ \\
\hline Male sex & $296(49.3)$ \\
\hline Age (years) mean (SD) & $64.6(17.6)$ \\
\hline \multicolumn{2}{|l|}{ Mortality } \\
\hline In-hospital mortality within 28-days & $75(12.5)$ \\
\hline Total mortality within 28 days & $90(15.0)$ \\
\hline \multicolumn{2}{|l|}{ Severity of sepsis syndrome } \\
\hline Sepsis & $344(57.3)$ \\
\hline Severe sepsis & $220(36.7)$ \\
\hline Septic shock & $36(6.0)$ \\
\hline \multicolumn{2}{|l|}{ Complaints at presentation* } \\
\hline Fever/cold chills & $385(64.2)$ \\
\hline Dyspnoea & $160(26.7)$ \\
\hline Gastrointestinal & $141(23.5)$ \\
\hline Confusion & $95(15.8)$ \\
\hline Urinary & $22(3.7)$ \\
\hline \multicolumn{2}{|l|}{ Comorbidity* } \\
\hline Malignancy & $243(40.5)$ \\
\hline with metastases & $74(12.3)$ \\
\hline Chemotherapy within 28 days & $60(10.0)$ \\
\hline Immunocompromised & $156(26.0)$ \\
\hline Pulmonary disease & $76(12.7)$ \\
\hline Cardiac disease & $155(25.8)$ \\
\hline Diabetes Mellitus & $105(17.5)$ \\
\hline Neurological disease & $102(17.0)$ \\
\hline \multicolumn{2}{|l|}{ Therapy } \\
\hline Antibiotics administered at ED & $386(64.3)$ \\
\hline Antibiotics administered at ward & $116(19.3)$ \\
\hline \multicolumn{2}{|l|}{ Unit of admittance } \\
\hline General ward & $548(91.3)$ \\
\hline $\mathrm{MCU}$ & $20(3.3)$ \\
\hline ICU & $32(5.3)$ \\
\hline
\end{tabular}


Receiver operating characteristic analysis showed that all scoring systems had good discriminative ability for 28-day both in-hospital and total mortality (Figure 1, Table 3). Except for the MEDS, the AUCs for all scores were somewhat lower for total 28-day mortality than for 28-day in-hospital mortality.

For total 28-day mortality, the MEDS had the highest AUC $(0.82,95 \% \mathrm{Cl}: 0.78-0.87)$, which was significantly higher than the AUCs of the RAPS and REMS. For 28-day in-hospital mortality, the MEDS and CURB-65 had the highest AUCs (0.82 for both), but only the difference between the AUC of the MEDS and RAMS was significant $(P=0.003)$.The AUCS for the CURB-65 in patients with community-acquired pneumonia were not significantly different from the AUCS of the MEDS in this patient group for both total and in-hospital 28-day mortality.

Figure 1 ROC curves for the five scoring systems; prediction of total 28-day mortality

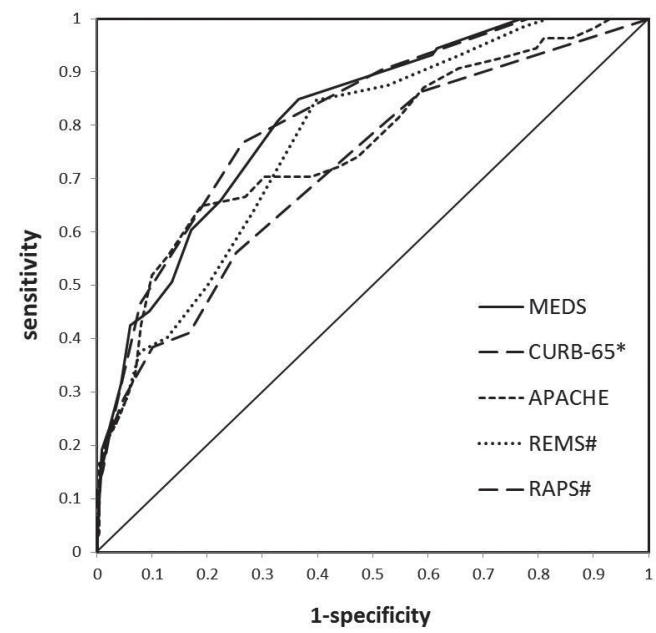

* calculated for all patients; ${ }^{*} p<0.005$, compared with AUC of the MEDS score

Table 3 AUCs for the 5 scoring systems

\begin{tabular}{|c|c|c|c|c|c|c|c|}
\hline Scoring system & $n$ & $\begin{array}{l}\text { in-hospi } \\
\text { (95\%Cl) }\end{array}$ & nortality & $\begin{array}{l}\text { p-value } \\
\#\end{array}$ & $\begin{array}{l}\text { total } \mathrm{m} \\
(95 \% \mathrm{Cl})\end{array}$ & ality & $\begin{array}{l}\text { p-value } \\
\#\end{array}$ \\
\hline MEDS (10) & 595 & 0.82 & $(0.77-0.86)$ & - & 0.82 & $(0.78-0.87)$ & \\
\hline CURB-65* (11) & 577 & 0.82 & $(0.77-0.87)$ & 0.911 & 0.78 & $(0.73-0.83)$ & 0.095 \\
\hline CURB-65 (11) & 222 & 0.77 & $(0.69-0.85)$ & 0.952 & 0.72 & $(0.63-0.80)$ & 0.125 \\
\hline APACHE II (12) & 256 & 0.76 & $(0.68-0.84)$ & 0.748 & 0.71 & $(0.64-0.79)$ & 0.196 \\
\hline RAPS (13) & 596 & 0.72 & $(0.66-0.79)$ & 0.003 & 0.70 & $(0.64-0.76)$ & $<0.001$ \\
\hline REMS (14) & 594 & 0.78 & $(0.72-0.83)$ & 0.127 & 0.74 & $(0.69-0.80)$ & 0.007 \\
\hline
\end{tabular}


Figure 2 shows the comparison between the observed mortality rates in our study and those reported in the original validation studies (expected mortality) for the MEDS, the CURB-65 and the APACHE II score. For the lowest two risk levels of the MEDS, observed mortality and expected mortality according to the original study corresponded well [10]. However, the observed mortality was roughly twice as high as expected for other levels of the MEDS score. Also for the CURB-65, observed mortality was increasingly higher than expected, but this was not as pronounced as for the MEDS [10, 11]. In contrast, for the APACHE II score, we found somewhat lower mortality rates for the higher risk levels than reported in the original study [12].

Figure 2 Comparison of observed mortality and mortality reported in the original validation studies [10-12]

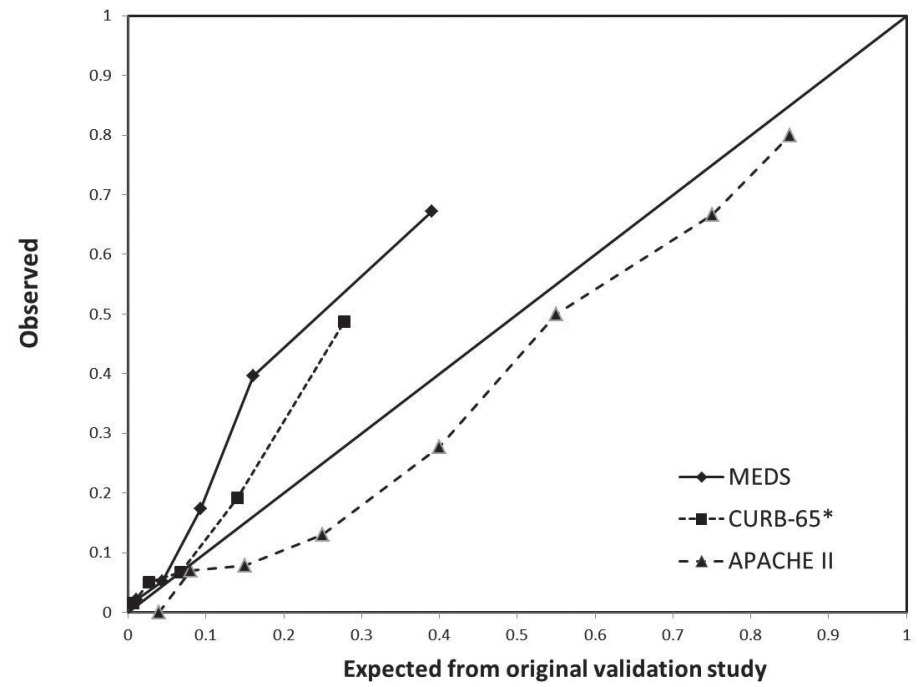

* calculated for all patients

Feasibility

The MEDS, REMS and RAPS could be calculated in $99 \%$ of all patients, the CURB- 65 in $96 \%$ and the APACHE II in 42\% (Table 4). Missing laboratory items were the main cause for incomplete scores (thrombocytes for MEDS; urea for CURB-65, and haematocrit and arterial blood gas for APACHE II). The total 28-day mortality rates for those patients for whom the scoring systems could be calculated and for the total group were almost equal for all scores (range $15.0 \%-15.6 \%$ ) except for the APACHE II, where the mortality was clearly higher than in the total group ( $25.4 \%$ vs. $15.0 \%$; $p<0.0001$ ).

We assessed the availability of all items necessary to calculate the different scores in subgroups on the basis of all patient characteristics listed in Table 2. Table 4 shows all items for which there was a statistically significant difference between subgroups for at least one of the scores. There were only clear differences for the subgroups on the basis 
of sepsis syndrome severity and for subgroups on the basis of two presenting complaints. The APACHE II score could be calculated more often for patients with increasing severity of sepsis $(23.8 \%, 64.5 \%$ and $88.9 \% ; \mathrm{P}=0.0001)$ and for patients with dyspnoea $(68.1 \%$ vs. $31.9 \%$ in other patients; $P<0.0001$ ) because a blood gas was analysed more frequently in such patients. In patients with and without fever, the APACHE could be calculated $35.6 \%$ and $55.3 \%(p<0.0001)$.

Table 4 Availability of items for calculations of the five scores: relation to the severity of sepsis and presenting complaints

\begin{tabular}{|c|c|c|c|c|c|}
\hline Scoring system & MEDS & CURB-65* & APACHE II & RAPS & REMS \\
\hline & n (\%) & n (\%) & n (\%) & n (\%) & n (\%) \\
\hline $\begin{array}{l}\text { All items available, } \\
\mathrm{n}(\%)\end{array}$ & $595(99.2)$ & $577(96.2)$ & $256(42.7)$ & $596(99.3)$ & $594(99.2)$ \\
\hline $\begin{array}{l}\text { Total 28-day } \\
\text { mortality (\%) }\end{array}$ & $89(15.0)$ & $90(15.6)$ & $65(25.4)^{\#}$ & $90(15.1)$ & $90(15.2)$ \\
\hline \multicolumn{6}{|l|}{ Missing items**, n (\%) } \\
\hline Arterial blood gas & - & - & $344(57.3)$ & - & - \\
\hline Thrombocytes & $5(0.8)$ & - & - & - & - \\
\hline Urea & - & $23(3.8)$ & - & - & - \\
\hline Haematocrit & - & - & $241(40.2)$ & - & - \\
\hline Other & $1(0.2)^{\mathrm{a}}$ & $0(0.0)$ & $0(0.0)$ & $4(0.7)^{b}$ & $5(0.8)^{\mathrm{b}, \mathrm{c}}$ \\
\hline \multicolumn{6}{|l|}{ Sepsis severity category } \\
\hline Sepsis $(n=344)$ & $340(98.8)$ & $324(94.2)$ & $82(23.8)^{\# \#}$ & $343(99.7)$ & $343(99.7)$ \\
\hline $\begin{array}{l}\text { Severe sepsis } \\
(n=220)\end{array}$ & $220(100.0)$ & 218 (99.1) & $142(64.5)^{\# \#}$ & $220(100.0)$ & $220(100.0)$ \\
\hline Septic shock $(n=36)$ & $35(97.2)$ & $35(97.2)$ & $32(88.9)^{\# \#}$ & $33(91.7)$ & 32 (88.9) \\
\hline \multicolumn{6}{|l|}{ Presenting complaints } \\
\hline Fever $(n=385)$ & $383(99.5)$ & $372(96.6)$ & $137(35.6)^{\#}$ & $383(99.4)$ & $382(99.2)$ \\
\hline Dyspnoea $(n=160)$ & $158(99.4)$ & 155 (96.9) & $109(68.1)^{\#}$ & 159 (99.4) & $159(99.4)$ \\
\hline
\end{tabular}

\section{Limitations}

Our study has some limitations. First, we depended on the notes of physicians to retrieve all the necessary information. The Glasgow Coma Scale (GCS) and respiratory rate were often not recorded exactly. These data were deduced from other recorded information, and although this method is less accurate in this respect, other authors used similar solutions for these problems $[16,19]$. Inaccurate data recording may have had an effect on decisions on the inclusion of patients and on their classification into sepsis severity categories. 
Second, our results on the APACHE II score stem from a subgroup of patients with more severe disease than the total group, as indicated by a higher mortality ( 25.4 vs. $15.0 \%)$ and more respiratory complaints (68.1 vs. $26.7 \%$ ). For now, it remains unclear what the accuracy of the APACHE II score would be when applied to the total group. Finally, like in most similar studies, the patients in this study did not receive standardized treatment. Therefore, the observed differences in mortality rate between subgroups will not only depend on the severity of disease at the moment of presentation at the ED but also on the treatment they received.

In terms of feasibility, we could retrieve data on comorbidity (metastatic cancer) and residence of the patients completely. It is possible that in other hospitals, this information is more difficult to retrieve, which may lead to lower feasibility.

\section{Discussion}

We investigated the predictive accuracy of risk stratification by the MEDS, CURB-65, APACHE II, RAPS and REMS, for 28-day mortality in consecutive adult patients with the sepsis syndrome, irrespective of the probable origin of the sepsis. These patients were generally referred by their general practitioner and admitted to the hospital by an internist after presentation at the ED. All five scoring systems discriminated well between patients who did and who did not die within 28 days, with AUCs ranging from 0.82 (MEDS) to 0.70 (RAPS). For total 28-day mortality, the MEDS had the highest AUC $(0.82,95 \% \mathrm{CI}: 0.78-0.87)$, which was significantly higher than the AUCs of the RAPS and REMS, but not compared with the CURB-65 and APACHE II. The AUCs for the different scoring systems were very similar to those reported by others $[10,11,13,14,16,19,21,22]$. Our finding that the MEDS score has a higher AUC than the REMS was reported in one other study before [19, 23].

The mortality rate in the present study was higher than in the original studies in which the MEDS and CURB-65 were internally validated $[10,11]$. It is unclear whether differences in patient selection or treatment between our hospital and the original hospitals explain these differences. The observed and predicted mortality corresponded well in the lowest risk levels, while the prediction became increasingly less accurate at higher estimated risks. In contrast, we found somewhat lower mortality rates using the APACHE II than those found in the original validation study [12]. The fact that the APACHE II was developed and validated in ICU patients, who are generally more seriously ill than our ED patient population, may explain these differences. Recalibration of the scoring systems before their use in our patient population seems necessary.

The MEDS, REMS, RAPS and CURB-65 turned out to be very feasible in our ED, as these could be calculated in almost all patients, although these scores were not used in the care for ED patients. The APACHE II score could be calculated less frequently (42.7\%) and is therefore presently less feasible for implementation at the ED. Scoring systems are considered useful for risk stratification of septic ED patients because they increase the awareness of physicians of the high risk of dying that patients with the sepsis syndrome carry [16, 18]. Improved awareness is expected to lead to more timely and appropriate treatment 
and hence improved prognosis [5-7]. The effect of rapid and aggressive treatment on mortality has already been shown in hospitals in which EGDT has been implemented for all patients with severe sepsis and septic shock $[8,9]$. In order to make implementation of the MEDS score for regular patient care at the ED more useful, there should be clarity about the treatment implications of the different levels of the sore instead on prediction of mortality. Future research should aim to determine appropriate cutoff levels to support treatment choices for individual patients.

\section{Conclusion}

The MEDS, CURB-65 and APACHE II scores had good discriminative ability for both 28-day mortality (either total or in-hospital) in ED patients with the sepsis syndrome. As the APACHE II was less feasible, the MEDS and CURB-65 are the most adequate risk stratification tools, but both need local recalibration to yield accurate estimates of mortality risk in our ED and probably in EDs of other hospitals. Future research should focus on the determination of appropriate cutoff levels of the MEDS for treatment decisions. 


\section{References}

1. Levy MM, Fink MP, Marshall JC, Abraham E, Angus D, Cook D, et al. 2001 SCCM/ESICM/ACCP/ATS/SIS International Sepsis Definitions Conference. Crit Care Med. 2003;31(4):1250-6.

2. Martin GS, Mannino DM, Eaton S, Moss M. The epidemiology of sepsis in the United States from 1979 through 2000. N EngI J Med. 2003;348(16):1546-54.

3. Rangel-Frausto MS, Pittet D, Costigan M, Hwang T, Davis CS, Wenzel RP. The natural history of the systemic inflammatory response syndrome (SIRS). A prospective study. JAMA. 1995;273(2):117-23.

4. Assuncao M, Akamine N, Cardoso GS, Mello PV, Teles JM, Nunes AL, et al. Survey on physicians' knowledge of sepsis: do they recognize it promptly? J Crit Care. 2010;25(4):545-52.

5. Rivers E, Nguyen B, Havstad S, Ressler J, Muzzin A, Knoblich B, et al. Early goal-directed therapy in the treatment of severe sepsis and septic shock. N Engl J Med. 2001;345(19):1368-77.

6. Jones AE, Focht A, Horton JM, Kline JA. Prospective external validation of the clinical effectiveness of an emergency department-based early goal-directed therapy protocol for severe sepsis and septic shock. Chest. 2007;132(2):425-32.

7. Puskarich MA, Marchick MR, Kline JA, Steuerwald MT, Jones AE. One year mortality of patients treated with an emergency department based early goal directed therapy protocol for severe sepsis and septic shock: a before and after study. Crit Care. 2009;13(5):R167.

8. Rivers EP, Coba V, Whitmill M. Early goal-directed therapy in severe sepsis and septic shock: a contemporary review of the literature. Curr Opin Anaesthesiol. 2008;21(2):128-40.

9. Levy MM, Dellinger RP, Townsend SR, Linde-Zwirble WT, Marshall JC, Bion J, et al. The Surviving Sepsis Campaign: results of an international guideline-based performance improvement program targeting severe sepsis. Intensive Care Med. 2010;36(2):222-31.

10. Shapiro NI, Wolfe RE, Moore RB, Smith E, Burdick E, Bates DW. Mortality in Emergency Department Sepsis (MEDS) score: a prospectively derived and validated clinical prediction rule. Crit Care Med. 2003;31(3):670-5.

11. Lim WS, van der Eerden MM, Laing R, Boersma WG, Karalus N, Town GI, et al. Defining community acquired pneumonia severity on presentation to hospital: an international derivation and validation study. Thorax. 2003;58(5):377-82.

12. Knaus WA, Draper EA, Wagner DP, Zimmerman JE. APACHE II: a severity of disease classification system. Crit Care Med. 1985;13(10):818-29.

13. Rhee KJ, Fisher CJ, Jr., Willitis NH. The Rapid Acute Physiology Score. Am J Emerg Med. 1987;5(4):278-82.

14. Olsson T, Terent A, Lind L. Rapid Emergency Medicine score: a new prognostic tool for in-hospital mortality in nonsurgical emergency department patients. J Intern Med. 2004;255(5):579-87.

15. Olsson T, Lind L. Comparison of the rapid emergency medicine score and APACHE II in nonsurgical emergency department patients. Acad Emerg Med. 2003;10(10):1040-8.

16. Howell MD, Donnino MW, Talmor D, Clardy P, Ngo L, Shapiro NI. Performance of severity of illness scoring systems in emergency department patients with infection. Acad Emerg Med. 2007;14(8):709-14.

17. Jones AE, Trzeciak S, Kline JA. The Sequential Organ Failure Assessment score for predicting outcome in patients with severe sepsis and evidence of hypoperfusion at the time of emergency department presentation. Crit Care Med. 2009;37(5):1649-54.

18. Goodacre S, Turner J, Nicholl J. Prediction of mortality among emergency medical admissions. Emerg Med J. 2006;23(5):372-5.

19. DeLong ER, DeLong DM, Clarke-Pearson DL. Comparing the areas under two or more correlated receiver operating characteristic curves: a nonparametric approach. Biometrics. 1988;44(3):837-45.

20. Crowe CA, Kulstad EB, Mistry CD, Kulstad CE. Comparison of severity of illness scoring systems in the prediction of hospital mortality in severe sepsis and septic shock. J Emerg Trauma Shock;3(4):342-7.

21. Shapiro NI, Howell MD, Talmor D, Donnino M, Ngo L, Bates DW. Mortality in Emergency Department Sepsis (MEDS) score predicts 1-year mortality. Crit Care Med. 2007;35(1):192-8.

22. Cildir E, Bulut M, Akalin H, Kocabas E, Ocakoglu G, Aydin SA. Evaluation of the modified MEDS, MEWS score and Charlson comorbidity index in patients with community acquired sepsis in the emergency department. Intern Emerg Med. 2013;8(3):255-60.

23. Chen CC, Chong CF, Liu YL, Chen KC, Wang TL. Risk stratification of severe sepsis patients in the emergency department. Emerg Med J. 2006;23(4):281-5. 



\section{Chapter 6}

\section{Predictive scoring in sepsis patients at the emergency department; the AbbMEDS performs best}

Asselina A. Roest, Mariëlle E.C. Bakker, Judith Stoffers. Michelle J.M. Hilderink, Yolande C. Keulemans, Coen D.A. Stehouwer, Patricia M. Stassen

Submitted 


\begin{abstract}
Purpose

Risk stratification scores identify sepsis patients with a high mortality risk. We investigated the ability to predict 28-day mortality of 14 risk stratification scores and two laboratory values and investigated the scores with high discriminatory value and feasibility regarding age-specific discriminatory value and calibration.
\end{abstract}

\title{
Methods
}

We retrospectively included a cohort of sepsis patients at the ED. We analysed the discriminatory value and feasibility of 14 scores: MEDS, abbMEDS, PIRO, SSS, SAPSII, SOFA, APACHEII, REMS, Shock Index, RAPS, CURB-65, PSI, NaURSE, MEWS and two laboratory values: CRP and WBC. We compared the scores with an AUC of $\geq 0.70$ and a feasibility of $>90 \%$ regarding discriminatory value in all patients and in four age categories $(<65, \geq 65$, $\geq 75$ and $\geq 85$ years) and calibrated these.

\section{Results}

In 1230 patients 28 -day mortality was $14.5 \%$. The abbMEDS was highly feasible (91.4\%) and had a higher AUC 0.78(95\% $\mathrm{Cl}: 0.75-0.82)$ than other scores with a high discriminatory value and feasibility: REMS, CURB-65 and NaURSE. The discriminatory value of abbMEDS was maintained in elderly patients. The abbMEDS was well calibrated.

\section{Conclusions}

AbbMEDS is most accurate in predicting 28-day mortality in sepsis patients at the ED and is highly feasible and well calibrated. Its discriminatory value is maintained in elderly patients. 


\section{Introduction}

\section{Background}

Sepsis is a major health care problem, which affects millions of people worldwide. The in-hospital mortality increases from $16 \%$ of patients with uncomplicated sepsis to $46 \%$ of patients with septic shock [1]. Underlining the importance to identify sepsis patients with the highest chance to die in an early stage is the finding that every hour of delay in administration of antibiotics in patients with septic shock was associated with an increase of mortality of $7.6 \%[2]$.

\section{Importance}

To identify sepsis patients with a high chance of dying, a predictive score is helpful. Several scores that quantify the severity of illness have been developed and validated in different populations and countries $[3,4]$. However, these scores have often been designed and validated in the same cohort, which may lead to an overestimation of their discriminatory value [5-7]. In addition, the scores have often not been calibrated [5, 8]. Further, although some of the scores include items regarding age, the predictive value of the scores have not been tested in elderly patients, who form a major part of the patients with sepsis. To improve the value of predictive scores, it is important to evaluate which score is best, regarding discriminatory value and feasibility, by comparing these scores with each other in external validation studies with large populations of sepsis patients and in different age categories.

Besides predictive scores, in daily practice, traditional inflammation markers such as Creactive protein (CRP) and white blood cell count (WBC) are used to assess severity of disease. Two studies reported that CRP was higher in survivors than in non-survivors in elderly patients $[9,10]$. The association between WBC and mortality has not yet been established [11,12]. Of course, a comparison between a single laboratory value and a complete score consisting of multiple items is not completely fair. However, because of their use in daily practice it is important to establish whether these inflammation markers can predict mortality in sepsis patients at all.

\section{Goals of this investigation}

The aim of our study was to investigate the discriminatory value and feasibility of severity scores and traditional inflammation markers (WBC and CRP) in predicting 28-day mortality in a sepsis population at the ED. Furthermore, we aimed to find out which of the scores performs best with respect to discriminatory value and feasibility. In addition, we aimed to investigate the age-specific discriminatory value of the best scores and to calibrate these scores with the original derivation studies. 


\section{Methods}

\section{Study design and setting}

This retrospective cohort study was performed in the Maastricht University Medical Center (MUMC+). This is a secondary and tertiary teaching hospital in the Netherlands. Yearly, approximately 23,000 patients visit our ED, of whom approximately 5,000 are assessed and treated by acute care internists and their residents. There are no ED physicians in our ED. Most patients are referred by a general practitioner and almost all acute admissions originate from the ED (for more information on the organization of EDs in the Netherlands, we refer to [13]). This study was approved by the Medical Ethics Committee of MUMC+.

\section{Selection of scores}

We performed a literature search in the PubMed database using the following terms; "Emergency Department", "Sepsis", "Severe Sepsis", "Septic shock", "Infection", "Systemic Inflammatory Response Syndrome", "Severity of Illness Index", "Mortality" and "Prognosis" in different combinations using both MESH and free terms. We found 18 scores, which could - in theory - be applied to a sepsis population at the ED (details in Table 1). We included sepsis-specific ED scores, ICU scores, non-disease specific ED scores and Other scores, such as disease-specific, transport- and bedside scores.

We included the following sepsis-specific ED scores: MEDS (Mortality in Emergency Department Sepsis), abbMEDS (abbreviated Mortality in Emergency Department Sepsis) and PIRO (Predisposition, Infection, Response and Organ failure), which have been developed specifically for sepsis patients at the ED, and the SSS (Sepsis Severity Score), which was developed in patients with severe sepsis and septic shock at the ED (Table 1) [5, 7, 14-16]. The SSS consists of 19 different items of which two are the same in our whole population (sepsis origin and geographic region), one item that is not applicable to our population (mechanical ventilation) and two items that are not regularly documented in our patients (response to fluids and presence of chills). We excluded the last three items from the SSS score and refer to it as modified SSS (mSSS). We further included three ICU scores that have previously been tested in sepsis patients at the ED: the SAPSII (Simplified Acute Physiology Score II), SOFA (Sequential Organ Failure Assessment) and APACHE II (Acute Physiology and Chronic Health Evaluation II) [6, 17-20]. The SAPSII score was included despite the fact that the score includes 2 items that are not applicable to (most) ED patients: details on mechanical ventilation and measurement of urinary output [17]. We excluded these items from the score, and in this manuscript, we will refer to SAPSII as modified SAPS II (mSAPSII).

Two non-disease-specific ED scores were included: REMS (Rapid Emergency Medicine Score) and the Shock Index [6, 21-23]. The other included scores (disease-specific, transport and bedside scores) have been developed in other populations but have also been tested in sepsis patients at the ED. These were the RAPS (Rapid Acute Physiology Score), CURB-65 (Confusion, Urea, Respiratory rate, Blood pressure and Age $>65$ ) and MEWS (Mo- 
dified Early Warning Score) [5, 6, 14, 24-26]. There are several, highly similar, early warning scores, but we chose to include the MEWS only, because this score is most frequently used in sepsis patients at the $\operatorname{ED}[27,28]$.

The PSI (Pneumonia severity index) was also included in the category Other scores, because this is a well-known predictive score in patients with pneumonia [29]. We also included the NaURSE (Sodium, Urea, Respiratory rate, Shock index in the Elderly), which is a new score with predictive ability in the acutely admitted oldest old and that in theory can be applied to sepsis patients at the ED [30].

We excluded four scores (Table 1). The MISSED score (Mortality in Severe Sepsis in the Emergency Department), a sepsis-specific score, was excluded because it includes two (out of a total of three) items which are not routinely measured in our sepsis population (international normalized ratio and albumin) [31]. Second, we excluded the MPM0-II score (Mortality Probability Models) [32], which was originally validated in an ICU population, but has also been used in sepsis patients at the ED [33]. However, the MPM0-II includes items like "resuscitation" and "mechanical ventilation", which are not applicable to our sepsis patients. In sum, the item "emergency ICU admission" (the same in all our patients) led to 3 out of $9 \mathrm{missing} /$ the same items in our population, thereby reducing its discriminatory value. Third, we excluded the MODS score (Multiple Organ Dysfunction Score), which is also an ICU-derived score, and includes items similar to the SOFA (Sepsis-related Organ Failure Assessment) score, but the score-specific item "pressure-adjusted heart rate" could not be measured in our population [18, 34]. Fourth, the APS (Acute Physiology Score) was excluded, because we included the APACHE II score which was derived from the APS score [35]. In addition, the APS score consists of 34 items, which makes the score not feasible for use at the ED. 


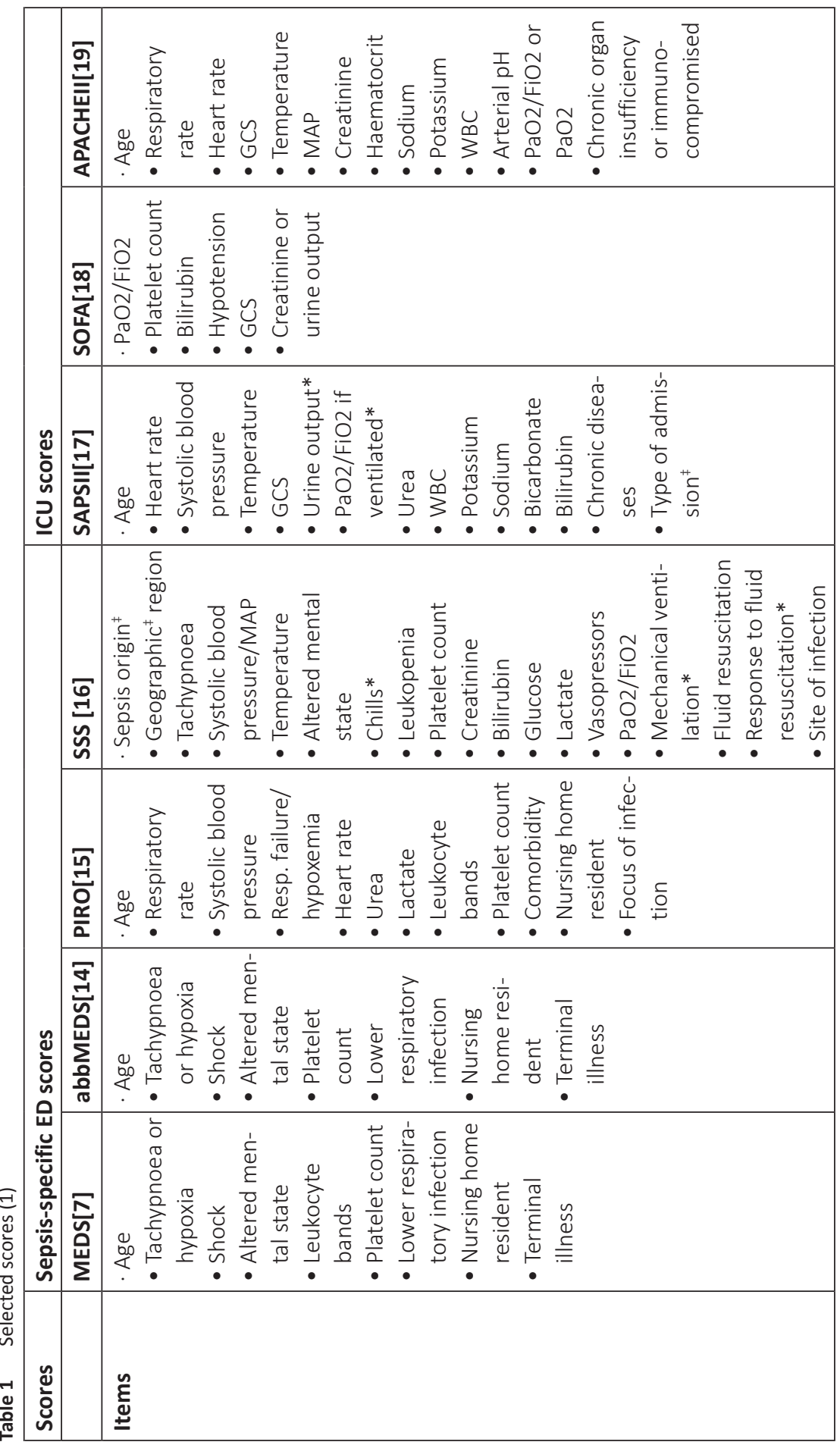




\begin{tabular}{|c|c|c|c|c|}
\hline 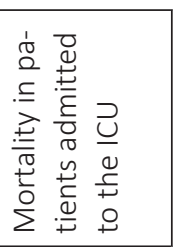 & 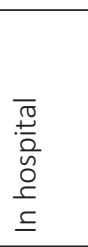 & $\begin{array}{l}\stackrel{0}{\hat{~}} \\
\text { ने }\end{array}$ & $\begin{array}{l}-1 \\
\hat{0}\end{array}$ & 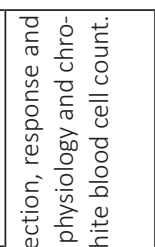 \\
\hline 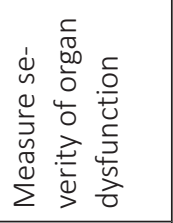 & $\stackrel{\text { வீं }}{\check{c}}$ & 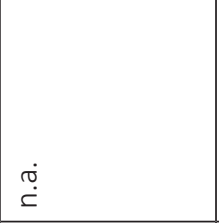 & 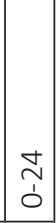 & 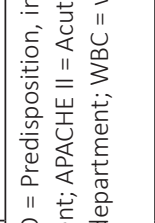 \\
\hline 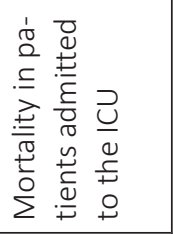 & 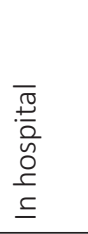 & 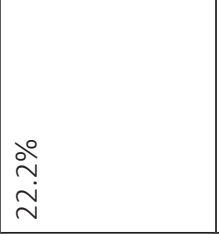 & 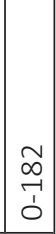 & 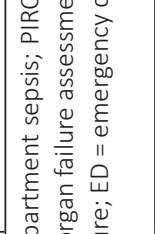 \\
\hline 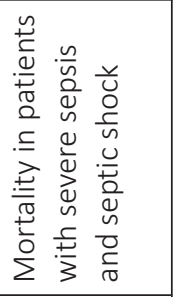 & \begin{tabular}{|l}
$\overline{0}$ \\
$\frac{\pi}{2}$ \\
$\overline{2}$ \\
0 \\
$\frac{1}{1}$ \\
$\underline{\underline{n}}$ \\
\end{tabular} & $\begin{array}{l}\stackrel{\circ}{\text { ஸे }} \\
\text { m }\end{array}$ & $\begin{array}{l}0 \\
\stackrel{y}{7} \\
0 \\
\end{array}$ & 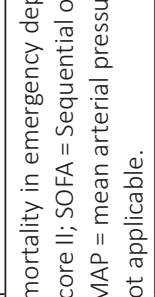 \\
\hline 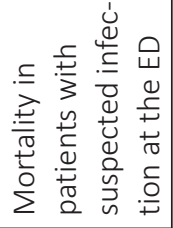 & 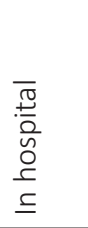 & 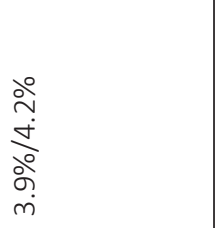 & $\begin{array}{c}m \\
m \\
\dot{d}\end{array}$ & 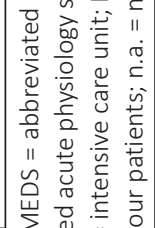 \\
\hline 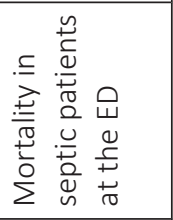 & 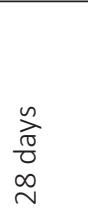 & 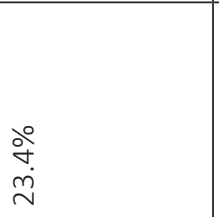 & 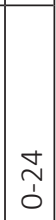 & 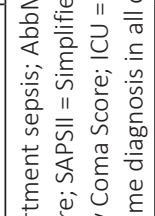 \\
\hline 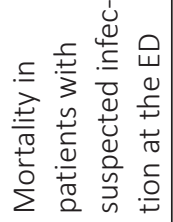 & 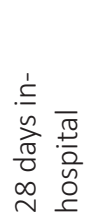 & 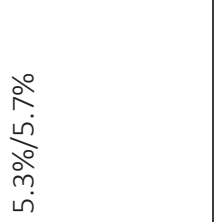 & $\begin{array}{c}\hat{\gamma} \\
\hat{1}\end{array}$ & 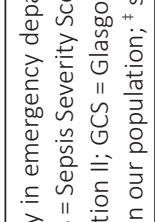 \\
\hline 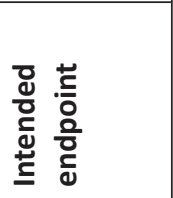 & $\begin{array}{l}\frac{2}{\frac{2}{5}} \\
\frac{t}{0} \\
\sum\end{array}$ & 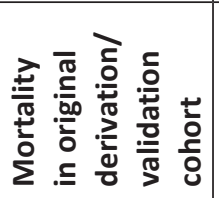 & 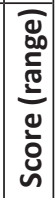 & 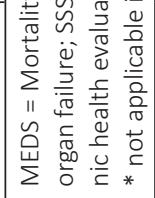 \\
\hline
\end{tabular}




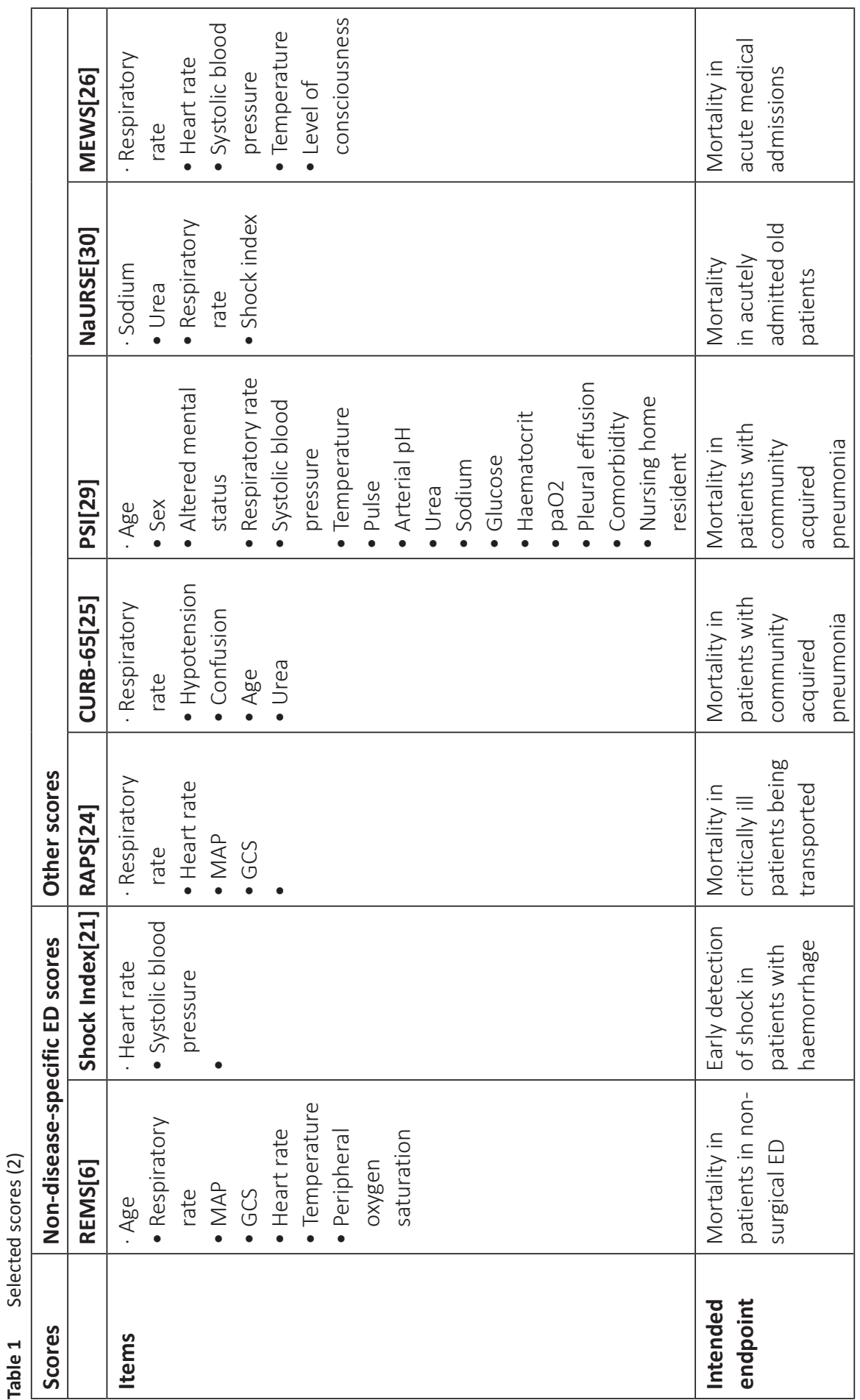




\begin{tabular}{|c|c|c|c|}
\hline $\begin{array}{l}\frac{0}{0} \\
\frac{1}{0} \\
8 \\
8\end{array}$ & $\begin{array}{c}\stackrel{\circ}{0} \\
\infty \\
\infty\end{array}$ & 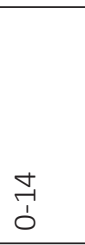 & 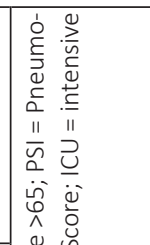 \\
\hline 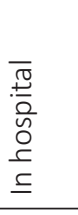 & $\begin{array}{l}\stackrel{\circ}{\circ} \\
\text { mे } \\
\text { - }\end{array}$ & fं & 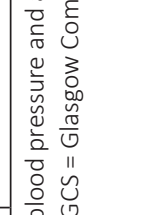 \\
\hline $\begin{array}{l}\text { 耐 } \\
\text { D } \\
\text { m }\end{array}$ & 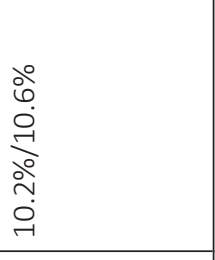 & 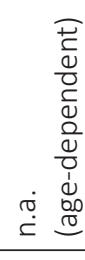 & 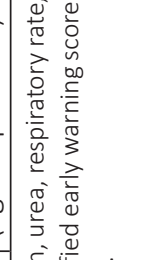 \\
\hline $\begin{array}{l}n \\
\frac{\pi}{0} \\
0 \\
0 \\
m\end{array}$ & 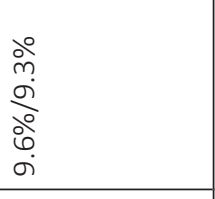 & 占 & 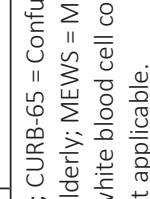 \\
\hline 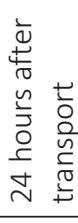 & $\begin{array}{l}\text { 今े } \\
\text { ते }\end{array}$ & $\begin{array}{l}0 \\
\stackrel{1}{0}\end{array}$ & 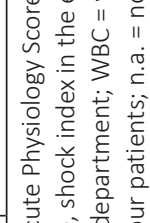 \\
\hline 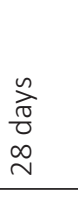 & 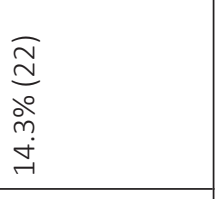 & 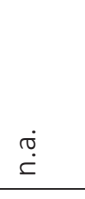 & 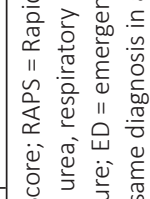 \\
\hline 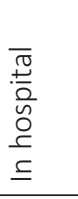 & 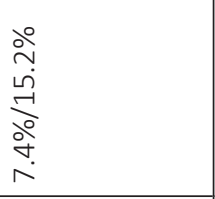 & $\begin{array}{l}\text { ô } \\
\text { ò }\end{array}$ & 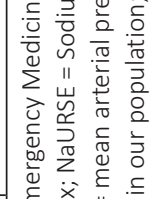 \\
\hline 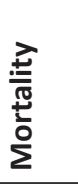 & 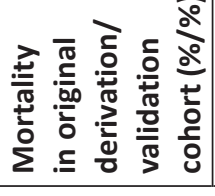 & 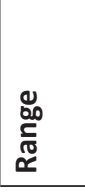 & 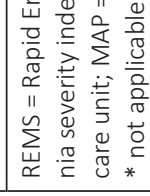 \\
\hline
\end{tabular}




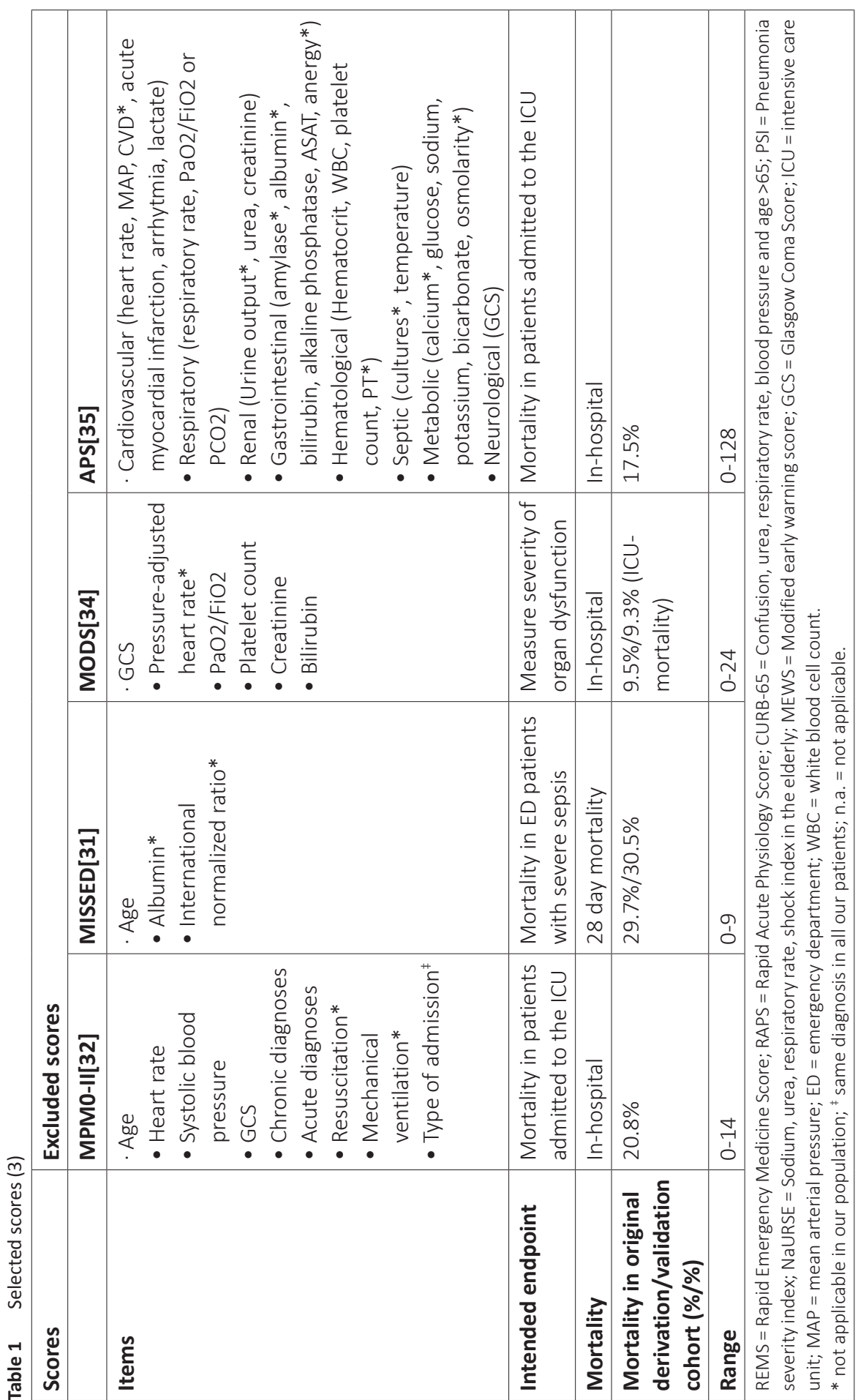




\section{Selection of participants}

We evaluated all ED charts of patients assessed at the ED and admitted to the hospital (including medium care and intensive care unit) by the internist. We included sepsis patients aged 18 years and older in a 28 month period (between August 1, 2009 and July 31, 2010 and between March 28, 2011 and July 31, 2012). Only the first ED visit was included in our analysis, to avoid confounding our dataset with repeated measures. The patients were followed up for 28 days or until death within 28 days. We excluded patients with incomplete follow-up ( $N=16)$.

The diagnosis sepsis was defined using international criteria at the ED [36, 37], i.e. suspicion of infection and 2 or more of 4 systemic inflammatory response syndrome (SIRS) criteria: 1 ) heart rate $>90 / \mathrm{min}, 2$ ) respiratory rate $>20 / \mathrm{min}$ or $\mathrm{pCO} 2<4.3 \mathrm{kPa}, 3$ ) temperature $<36.0^{\circ} \mathrm{C}$ or $>38.3^{\circ} \mathrm{C}$, 4) leukocytes $<4 \times 109 / \mathrm{L}$ or $>12 \times 109 / \mathrm{L}$ or $>10 \%$ leukocyte bands. The diagnosis sepsis was retrieved from the charts by one investigator and confirmed by another and in case of doubt, a third investigator decided on the issue. Part of our patients $(\mathrm{N}=664,45.8 \%)$ have been included in another study [38].

\section{Methods and measurements}

We used standardized extraction forms to retrieve the data from the charts. From these charts, demographic data, medical history, vital signs, laboratory values, suspected focus of infection and total scores of the scores at the ED were retrieved. All-cause mortality within 28 days, both in- and out of-hospital, was retrieved from the charts as well, and if necessary, by contacting the general practitioner. To complete missing data, we calculated the following missing items from other available values in the chart. We converted the $\mathrm{PaO} 2 / \mathrm{FiO} 2$ ratio into a $\mathrm{SpO} / \mathrm{FiO} 2$ ratio after calculating the $\mathrm{FiO} 2$ delivered per oxygen delivery device $(39,40)$. We also converted haemoglobin values into haematocrit [41]. Some items, such as respiratory rate, were not always explicitly noted in the chart. To complete these items, we converted text notes into numerical data (Appendix, Table 1). When (other) data were missing, we completed the scores by assuming that the missing data were normal.

\section{Outcomes}

To assess the discriminatory value of severity scores in predicting 28-day mortality, we calculated the area under the receiver operator characteristics curve (AUC-ROC) for every score and the two laboratory values (CRP/WBC). In our manuscript, we refer to AUC-ROC as AUC. We analysed the Shock Index both as a total score and by using two cut-off values of $>0.7$ and $>1.0(22,23)$. Likewise, for CRP, we analysed the value as a total score and using two cut-off points of 70 and $100 \mathrm{mg} / \mathrm{L}$ [9]. We further tested the combination of abnormal WBC and CRP $>70 \mathrm{mg} / \mathrm{L}$.

To assess the feasibility of the scores in our sepsis population at the ED, we calculated the proportion of scores that could be filled out completely. In addition, we retrieved the main reasons for missing data. To assess whether there were differences between patients 
for whom scores could be calculated completely and for those for whom scores were incomplete, we retrieved 28-day mortality in these two groups.

For the best scores, defined as scores with an AUC $\geq 0.70$ and a feasibility $>90 \%$, we investigated differences in discriminatory value among these scores. To assess whether the discriminatory value of the best scores is age-dependent, we investigated this using different cut-off points $(<65, \geq 65, \geq 75$ and $\geq 85$ years of age). We compared the discriminatory value of the best scores with each other within each age group. Last, these best scores were calibrated by comparing the mortality rates we found per predefined risk category with mortality rates reported in the original studies. For the REMS and NaURSE score, we combined the two highest risk categories, because the number of patients in the high risk category was small.

\section{Analysis}

All statistical analyses were processed with IBM SPSS Statistics 20.0. for Windows (IBM, Armont, New York, USA, 2011) and MedCalc for Windows, version 12.4.0.0 (MedCalc Software, Mariakerke, Belgium). Means and medians were calculated with standard deviation $(S D)$ and interquartile ranges (IQR), respectively. AUCs were calculated for all scores. Differences between the AUCs were calculated using the DeLong test [42]. P values lower than 0.05 were considered significant.

\section{Results}

\section{Characteristics of study subjects}

In the 28-month study period, 1451 patients with sepsis were assessed by the internist at the ED (Figure 1). In total, 221 patients (15.2\%) were excluded because of subsequent visits $(205,14.1 \%)$ and because of incomplete follow up $(16,1.1 \%)$. Therefore, the study population consisted of 1230 patients.

Pneumonia was the most commonly suspected focus of infection (33.2\%). Mortality within 28 days was $14.5 \%$ (Table 2 ).

Figure 1 Flowchart of study population

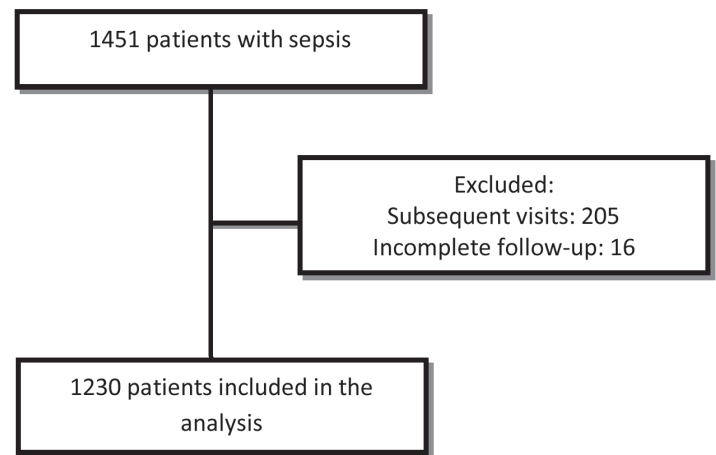


Table 2 Patient characteristics and outcome

\begin{tabular}{|c|c|}
\hline N (\%) or median (IQR) & Patients ( $\mathrm{N}=1230)$ \\
\hline \multicolumn{2}{|l|}{ Demographics } \\
\hline Age (mean, SD, years) & $64.5(17.3)$ \\
\hline$<65$ & $563(45.8)$ \\
\hline$\geq 65$ & $667(54.2)$ \\
\hline$\geq 75$ & $400(32.5)$ \\
\hline$\geq 85$ & $152(12.4)$ \\
\hline Male & $598(48.6)$ \\
\hline Nursing home resident & $99(8.0)$ \\
\hline \multicolumn{2}{|l|}{ Comorbidity } \\
\hline Malignancy & $445(36.2)$ \\
\hline Renal disease & $240(19.5)$ \\
\hline Diabetes & $223(18.1)$ \\
\hline Chronic obstructive pulmonary disease & $107(8.7)$ \\
\hline Congestive heart failure & $82(6.7)$ \\
\hline \multicolumn{2}{|l|}{ Suspected focus of infection } \\
\hline Pneumonia & $411(33.4)$ \\
\hline Urinary tract & $310(25.2)$ \\
\hline Gastro-enteritis & $192(15.6)$ \\
\hline Skin & $96(7.8)$ \\
\hline Hepatobiliary & $88(7.2)$ \\
\hline Other & 133(10.8) \\
\hline \multicolumn{2}{|l|}{ Sepsis severity } \\
\hline Sepsis & $671(54.6)$ \\
\hline Severe sepsis & $455(37.0)$ \\
\hline Septic shock & $104(8.5)$ \\
\hline \multicolumn{2}{|l|}{ Total score } \\
\hline \multicolumn{2}{|l|}{ Sepsis specific ED scores } \\
\hline MEDS & $5(3-8)$ \\
\hline abbMEDS & $5(3-8)$ \\
\hline PIRO & $8(6-13)$ \\
\hline mSSS & $28(21-36)$ \\
\hline \multicolumn{2}{|l|}{ ICU scores } \\
\hline mSAPSII & $27(21-34)$ \\
\hline SOFA & $2(0-3)$ \\
\hline APACHE II & $13(9-17)$ \\
\hline
\end{tabular}




\begin{tabular}{|l|r|}
\hline N (\%) or median (IQR) & Patients (N=1230) \\
\hline Non-disease-specific ED scores & $7(5-9)$ \\
\hline REMS & $0.80(0.67-0.96)$ \\
\hline Shock Index & $2(0-3)$ \\
\hline Other scores & $2(1-3)$ \\
\hline RAPS & $98(73-126)$ \\
\hline CURB-65 & $1(0-1)$ \\
\hline PSI & $3(2-4)$ \\
\hline NaURSE & $109(42-220)$ \\
\hline MEWS & $12.7(6.7-17.0)$ \\
\hline Laboratory values & $7(3-12)$ \\
\hline CRP & $27(2.2)$ \\
\hline WBC & $53(4.3)$ \\
\hline Outcomes & $178(14.5)$ \\
\hline Length of hospital stay, days & \\
\hline Medium care admission & \\
\hline Intensive care admission & \\
\hline Mortality within 28 days & \\
\hline $\begin{array}{l}\text { SD= standard deviation; IQR = interquartile range; CRP = C-reactive pro- } \\
\text { tein; WBC = White blood cell count. }\end{array}$ & \\
\hline
\end{tabular}

\section{Main results}

\section{Discriminatory value}

The AUCs for predicting 28-day mortality of the scores are shown in Table 3. All the sepsisspecific ED scores had an AUC > 0.70. Of the ICU scores, mSAPS II had the highest AUC $(0.77(95 \% \mathrm{Cl} 0.74-0.81))$. From the non-disease-specific and the Other scores, the PSI and NaURSE had the highest AUC $(0.79(95 \% \mathrm{Cl} 0.75-0.82)$ and $0.73(95 \% \mathrm{Cl} 0.68-0.77)$, respectively). The AUCs for the laboratory values were $0.62(0.57-0.66)$ for CRP and 0.55 (0.51$0.60)$ for WBC. 
Table 3 Discriminatory value and feasibility per score and two laboratory values

\begin{tabular}{|c|c|c|c|}
\hline & \multirow{2}{*}{$\begin{array}{l}\text { Discriminatory value } \\
\text { AUC }(95 \% \mathrm{Cl}) \\
(\mathrm{N}=1230)\end{array}$} & \multicolumn{2}{|l|}{ Feasibility } \\
\hline & & $\begin{array}{l}\text { Complete scores } \\
(\%)\end{array}$ & $\begin{array}{l}\text { 28-day mortality } \\
\text { in those with } \\
\text { complete score (\%) }\end{array}$ \\
\hline \multicolumn{4}{|c|}{ Sepsis-specific ED scores } \\
\hline MEDS & $0.78(0.75-0.82)$ & 74.9 & 14.4 \\
\hline abbMEDS & $0.78(0.75-0.82)$ & 91.4 & 14.9 \\
\hline PIRO & $0.75(0.71-0.79)$ & 15.8 & 26.3 \\
\hline $\mathrm{mSSS}$ & $0.72(0.68-0.77)$ & 7.0 & 19.8 \\
\hline \multicolumn{4}{|l|}{ ICU scores } \\
\hline mSAPS II & $0.77(0.74-0.81)$ & 22.5 & 30.4 \\
\hline SOFA & $0.70(0.66-0.74)$ & 8.1 & 23.0 \\
\hline APACHE II & $0.72(0.68-0.76)$ & 33.7 & 23.2 \\
\hline \multicolumn{4}{|c|}{ Non-disease-specific ED scores } \\
\hline REMS & $0.70(0.66-0.74)^{*}$ & 96.1 & 14.1 \\
\hline Shock Index & $0.58(0.53-0.63)$ & 99.8 & 14.4 \\
\hline Shock Index $>0.7$ & $0.51(0.47-0.56)$ & & \\
\hline Shock Index $>1.0$ & $0.57(0.53-0.62)$ & & \\
\hline \multicolumn{4}{|l|}{ Other scores } \\
\hline RAPS & $0.67(0.62-0.71)$ & 99.2 & 14.1 \\
\hline CURB-65 & $0.72(0.68-0.76)^{*}$ & 94.6 & 14.3 \\
\hline PSI & $0.79(0.75-0.82)$ & 24.1 & 23.6 \\
\hline NaURSE & $0.73(0.68-0.77)^{*}$ & 96.7 & 14.6 \\
\hline MEWS & $0.66(0.61-0.70)$ & 98.0 & 13.9 \\
\hline \multicolumn{4}{|l|}{ Laboratory values } \\
\hline CRP & $0.62(0.57-0.66)$ & 99.6 & 14.5 \\
\hline $\mathrm{CRP}>70 \mathrm{mg} / \mathrm{L}$ & $0.57(0.53-0.61)$ & & \\
\hline $\mathrm{CRP}>100 \mathrm{mg} / \mathrm{L}$ & $0.59(0.54-0.63)$ & & \\
\hline $\begin{array}{l}\text { WBC }<4 \text { of }>12 \\
10 \mathrm{E} 9 / \mathrm{L}\end{array}$ & $0.55(0.51-0.60)$ & 99.8 & 14.4 \\
\hline $\begin{array}{l}\text { Abnormal WBC } \\
\text { and } C R P>70 \mathrm{mg} / \mathrm{L}\end{array}$ & $0.58(0.53-0.62)$ & 99.3 & 14.5 \\
\hline
\end{tabular}


Chapter 6

Feasibility

The abbMEDS was the most feasible sepsis-specific ED score (91.4\%, Table 3). The ICU-scores were not feasible with a complete score ranging between 8.1-33.7\%. The non-diseasespecific ED scores and Other scores were feasible, except for the PSI (complete in 24.1\%). The scores that were most often complete and thus feasible were in general based on vital parameters and/or demographic data. The main reason of missing data were missing laboratory values, especially arterial blood gasses and lactate (Appendix, Table 4). Mortality in patients for whom the non-feasible scores could be completed was higher than in the total study cohort.

Comparison of the best scores

The four scores with an AUC of $\geq 0.70$ and a complete score $>90 \%$ were the sepsis-specific score abbMEDS, the non-disease-specific ED score REMS and two Other scores: the CURB65 and NaURSE (Table 3). The AUC of the sepsis specific ED score abbMEDS was highest (0.78) and significantly higher than that of the REMS, CURB-65 and NaURSE (AUC 0.70, 0.72 and 0.73 , respectively).

Age-specific discriminatory value of the four best scores

The discriminatory value of the sepsis specific score abbMEDS was highest within all age groups and significant in 3 out of 4 age groups compared to the REMS and the CURB-65 (Table 4). Both the abbMEDS and the NaURSE performed well in both younger and in older patients. Between these two scores, there was no difference in discriminatory value within the four age groups.

Table 4 Comparison of discriminatory value within each age group

\begin{tabular}{|c|c|c|c|c|}
\hline AUC $(95 \% \mathrm{Cl})$ & $\begin{array}{l}\text { Age }<65 \text { years } \\
N=563\end{array}$ & $\begin{array}{l}\text { Age } \geq 65 \text { years } \\
N=667\end{array}$ & $\begin{array}{l}\text { Age } \geq 75 \text { years } \\
N=400\end{array}$ & $\begin{array}{l}\text { Age } \geq 85 \text { years } \\
\mathrm{N}=152\end{array}$ \\
\hline abbMEDS & $0.79(0.72-0.87)$ & $0.73(0.65-0.78)$ & $0.77(0.72-0.83)$ & $0.79(0.71-0.87)$ \\
\hline REMS & $0.66(0.57-0.74)^{*}$ & $0.64(0.59-0.70)^{* *}$ & $0.65(0.58-0.71)^{\ddagger}$ & $0.71(0.62-0.80)$ \\
\hline CURB-65 & $0.65(0.55-0.74) *$ & $0.68(0.63-0.73)$ & $0.66(0.60-0.72)^{*}$ & $0.67(0.58-0.76)^{5}$ \\
\hline NaURSE & $0.72(0.63-0.80)$ & $0.71(0.66-0.76)$ & $0.74(0.68-0.79)$ & $0.78(0.70-0.86)$ \\
\hline
\end{tabular}

\section{Calibration of the four best scores}

We calibrated the abbMEDS, the REMS, the CURB-65 and the NaURSE with the derivation studies $[6,14,25,30]$ (Figure 2, Appendix, Table 5). The observed mortality (per predefined risk group in this study) and expected mortality (reported in the derivation studies) 
corresponded well for the three risk categories of abbMEDS, with a mortality of $3.8 \mathrm{vs.}$ $1.6 \%$ in the lowest predefined risk group and similar mortality rates in the other two risk categories. For the nine risk categories of the REMS in our study, the observed mortality was higher in the low and intermediate risk categories. Mortality in all three risk categories of the CURB-65 was higher than expected. For the four risk categories of the NaURSE, the observed mortality in the highest risk category was lower than expected ( 44.8 vs $83.4 \%$ ), while mortality in the lower risk categories corresponded well.

Figure 2 Comparison of observed mortality and expected mortality (original derivation studies) per predefined risk category

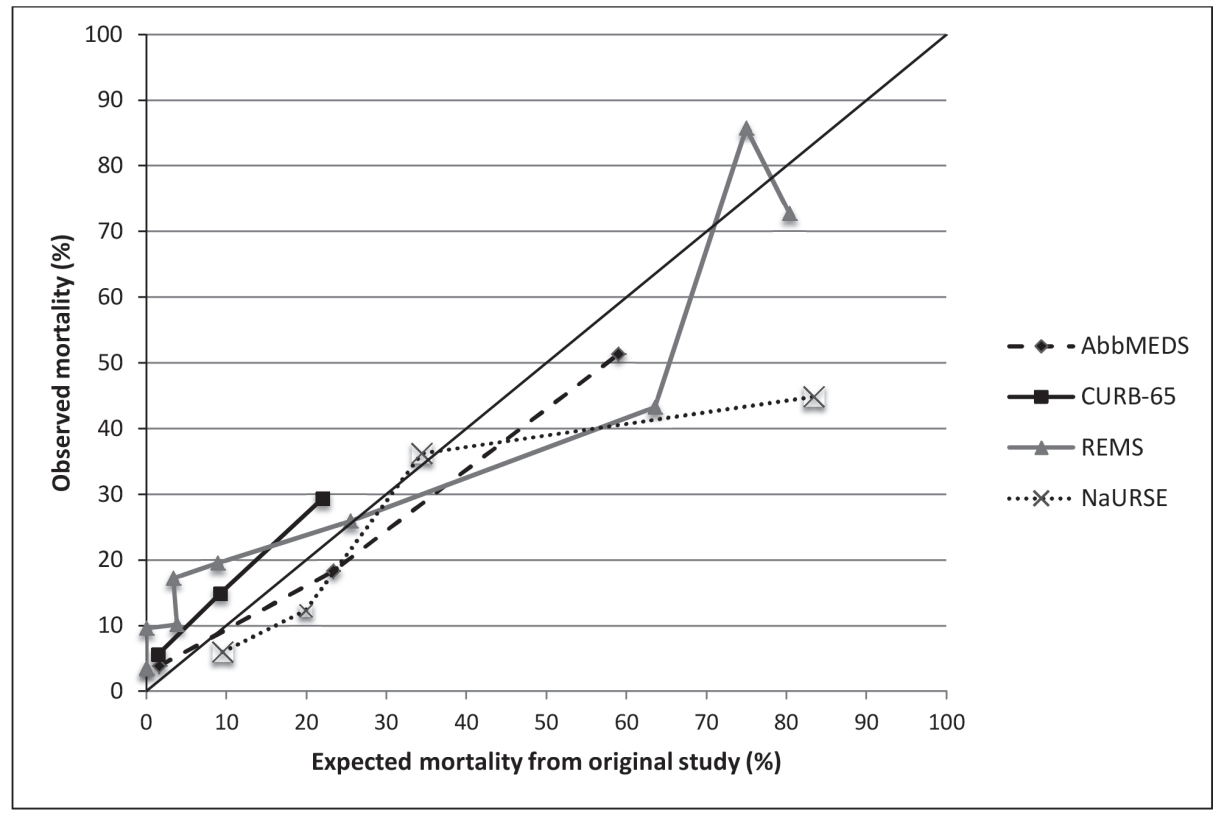

AbbMEDS = abbreviated mortality in emergency department sepsis; CURB-65 = Confusion, urea, respiratory rate, blood pressure and age $>65$; REMS = Rapid Emergency Medicine Score; NaURSE = Sodium, urea, respiratory rate, shock index in the elderly.

\section{Discussion}

In our large retrospective cohort study, we investigated the ability of fourteen severity of illness scores and two traditional inflammation markers to predict 28-day mortality in sepsis patients at the ED. The abbMEDS, REMS, CURB-65 and NaURSE all had high discriminatory value (AUC $\geq 0.70$ ) and were highly feasible (>90\%), the AbbMEDS turned out to have the highest AUC (0.78). In addition, the abbMEDS was well calibrated. Within each of the four age groups ( $<65, \geq 65, \geq 75$ and $\geq 85$ years of age), both abbMEDS and NaURSE performed better than REMS and CURB-65 and the discriminatory value of abbMEDS remained high in elderly patients. 
The sepsis-specific ED score abbMEDS was very feasible (complete score: $91.4 \%$ ) and had high discriminatory value. Its predictive value was somewhat lower than in the original study (AUC 0.82) [14]. In addition, the abbMEDS was well calibrated, in contrast to the REMS and CURB-65 that were less well calibrated in the lowest risk categories. Especially in the low risk group, it is important to be able to differentiate between low- and high-risk patients, because these patients might be safely treated as outpatients or with narrowspectrum antibiotics.

The non-disease-specific ED score REMS is very feasible (96.1\%), but has an AUC of 0.70, which is lower than in the original study (AUC 0.91) [6]. However, the original population consisted of both nonsurgical ICU and ED patients instead of an ED population with sepsis. In addition, its derivation and validation were performed in the same population, which can lead to a higher (than realistic) discriminatory value. From the Other scores, the CURB-65 and NaURSE had an AUC of $\geq 0.70$ and a complete score of $>90 \%$. The CURB- 65 score was developed in a population of patients with community-acquired pneumonia, and later validated in an ED population with suspected infection [5]. The NaURSE is a relatively new score specially designed for the acutely admitted elderly patient (median age: 93.5 years) [30]. The NaURSE was well calibrated in the low risk category, but in the high risk category, the observed mortality was much lower than expected (44.8 vs $83.4 \%$ )[30]. This may be explained by the lower mean age of our patients, because young patients have a higher chance of surviving the same severe disease than older patients. With regard to age-specific discriminatory value of the abbMEDS, although the AUC was highest in the age group $<65$ years (AUC 0.79 ), the discriminatory value of the abbMEDS increased per age group above 65 years from 0.73 to 0.79 in the group $\geq 85$ year. In several age groups, the discriminatory value of the abbMEDS and NaURSE was significantly higher than that of the REMS and CURB-65, reflecting the lower discriminatory value of the REMS and CURB-65 in the whole group.

Ten scores were less valuable at the ED. Some scores were not feasible. These were the sepsis-specific ED scores MEDS, PIRO and mSSS (complete scores: 74.9,15.8 and 7.0\%, respectively) and the three ICU-scores (complete scores ranging between 8.1-33.7\%). In addition, the ICU-scores were complete only in the sickest patients, which is reflected by the higher than average mortality (23.0-30.4\% vs $14.5 \%)$ in the group with complete scores.

Other scores had low discriminatory value. Among these was the non-disease-specific ED score, the Shock Index (AUC 0.58), irrespective of the use of different cut-off points. One study showed an AUC of 0.89 using a cut-off point of $\geq 1.0$ [23]. However, that study was performed in a small group of patients with severe sepsis and septic shock and a mortality of $54 \%$. This was also true for the two laboratory values. With regard to CRP, one study described CRP as a prognostic marker in elderly patients with a median value at admission of $70 \mathrm{mg} / \mathrm{L}$ [10]. However, the discriminatory value of WBC has also not been established by others either [11, 12]. Of all the scores we investigated, only the SSS includes a (low) WBC as a prognostic marker [16]. The low predictive value of both laboratory tests is not surprising; it is not expected that the course of a complex disease such as sepsis can be predicted by a simple blood test.

Our AUCs are in general slightly lower than in the original studies. This may be explained by differences in patient selection, setting and outcome measures. Our study population con- 
sisted of a sepsis population at the ED, while some scores were developed in a population with suspected infection, an ICU population, a general ED population or a population with a specific disease, like pneumonia $[6,7,19,25,29]$. Further, our outcome measure was overall 28-day mortality both in- and out-hospital, while other studies used only in-hospital mortality. $[5,7,17,19]$. Moreover, the scores were often derived and validated in the same population, thereby overestimating their discriminatory value $[6,7,16]$.

The main reason for incomplete scores in most cases was missing laboratory values. This can in part be explained by the retrospective nature of our study. However, it will not be feasible or desirable to perform all tests to complete the scores. For instance, a blood gas analysis is not necessary in every patient, is expensive and can cause complications such as false aneurysms [43].

In our view, instead of developing new scores, the quality of the current scores should be expanded. As a first step, we validated fourteen severity scores in predicting 28-day mortality in a large population of sepsis patients. In our study, we find four scores with a good discriminatory value and feasibility, namely the abbMEDS, REMS, CURB-65 and NaURSE. To further expand the quality of these scores, prospective multicentre studies are necessary with consistent and well-defined populations and outcome measures. A sepsis-specific ED score would ideally be part of a clinical decision support system that guides antibiotic treatment and disposition. Eventually, this may lead to a higher level of care in sepsis patients.

\section{Limitations}

These data stem from a single centre ED, but our study population is large and we compared more scores in the same population than ever reported before (as far as we know). Due to the retrospective nature of our study, we can only rely on documented data, which can lead to misclassification. However, the main factors we studied (vital signs, laboratory values and mortality) are less susceptible to misclassification and we only lost 16 patients to follow-up. Further, our study was performed in sepsis patients who were assessed and treated by the internist, and therefore our findings may not be applicable to all sepsis patients at the ED. Last, we modified two scores, the SSS and SAPSII, because these included items that are not applicable in an ED population.

\section{Conclusion}

In sepsis patients at the ED, the abbMEDS has the highest discriminatory value in predicting 28-day mortality, has a high feasibility and is well calibrated. This high discriminatory value of the AbbMEDS is maintained in elderly patients. The abbMEDS performs better than the other three scores with a high discriminatory value and feasibility, namely REMS, CURB-65 and NaURSE. ICU scores are not feasible at the ED due to the high number of missing data. The traditional inflammation markers CRP and WBC have no discriminatory value. 


\section{References}

1. Rangel-Frausto MS, Pittet $D$, Costigan M, Hwang T, Davis CS, Wenzel RP. The natural history of the systemic inflammatory response syndrome (SIRS). A prospective study. JAMA 1995;273:117-23.

2. Kumar A, Roberts D, Wood KE, Light B, Parrillo JE, Sharma S, et al. Duration of hypotension before initiation of effective antimicrobial therapy is the critical determinant of survival in human septic shock. Crit Care Med 2006;34:1589-96.

3. Challen K, Goodacre SW. Predictive scoring in non-trauma emergency patients: a scoping review. Emerg Med J 2011;28:827-37.

4. Calle P, Cerro L, Valencia J, Jaimes F. Usefulness of severity scores in patients with suspected infection in the emergency department: a systematic review. J Emerg Med 2012;42:379-91.

5. Howell MD, Donnino MW, Talmor D, Clardy P, Ngo L, Shapiro NI. Performance of severity of illness scoring systems in emergency department patients with infection. Acad Emerg Med 2007;14:709-14.

6. Olsson T, Lind L. Comparison of the rapid emergency medicine score and APACHE II in nonsurgical emergency department patients. Acad Emerg Med 2003;10:1040-8.

7. Shapiro NI, Wolfe RE, Moore RB, Smith E, Burdick E, Bates DW. Mortality in Emergency Department Sepsis (MEDS) score: a prospectively derived and validated clinical prediction rule. Crit Care Med 2003;31:670-5.

8. Chen YX, Li CS. Risk stratification and prognostic performance of the predisposition, infection, response, and organ dysfunction (PIRO) scoring system in septic patients in the emergency department: a cohort study. Crit care 2014;18:R74.

9. Povoa P. C-reactive protein: a valuable marker of sepsis. Intensive Care Med 2002;28:235-43.

10. Cox ML, Rudd AG, Gallimore R, Hodkinson HM, Pepys MB. Real-time measurement of serum C-reactive protein in the management of infection in the elderly. Age Ageing 1986;15:257-66.

11. Suberviola B, Castellanos-Ortega A, Gonzalez-Castro A, Garcia-Astudillo LA, Fernandez-Miret B. Prognostic value of procalcitonin, C-reactive protein and leukocytes in septic shock. Med Intensiva 2012;36:177-84.

12. Magrini L, Gagliano G, Travaglino F, Vetrone F, Marino R, Cardelli P, et al. Comparison between white blood cell count, procalcitonin and $C$ reactive protein as diagnostic and prognostic biomarkers of infection or sepsis in patients presenting to emergency department. Clin Chemistry Lab Med 2014;52:1465-72.

13. Thijssen WA, Giesen PH, Wensing M. Emergency departments in The Netherlands. Emerg Med J 2012;29:6-9.

14. Vorwerk C, Loryman B, Coats TJ, Stephenson JA, Gray LD, Reddy G, et al. Prediction of mortality in adult emergency department patients with sepsis. Emerg Med J 2009;26:254-8.

15. Howell MD, Talmor D, Schuetz P, Hunziker S, Jones AE, Shapiro NI. Proof of principle: the predisposition, infection, response, organ failure sepsis staging system. Crit Care Med 2011;39:322-7.

16. Osborn TM, Phillips G, Lemeshow S, Townsend S, Schorr CA, Levy MM, et al. Sepsis severity score: an internationally derived scoring system from the surviving sepsis campaign database*. Crit Care Med 2014;42:1969-76.

17. Le Gall JR, Lemeshow S, Saulnier F. A new Simplified Acute Physiology Score (SAPS II) based on a European/North American multicenter study. JAMA 1993;270:2957-63.

18. Vincent JL, Moreno R, Takala J, Willatts S, De Mendonca A, Bruining H, et al. The SOFA (Sepsis-related Organ Failure Assessment) score to describe organ dysfunction/failure. On behalf of the Working Group on Sepsis-Related Problems of the European Society of Intensive Care Medicine. Intensive Care Med 1996;22:707-10.

19. Knaus WA, Draper EA, Wagner DP, Zimmerman JE. APACHE II: a severity of disease classification system. Crit Care Med 1985;13:818-29.

20. Kofoed K, Eugen-Olsen J, Petersen J, Larsen K, Andersen O. Predicting mortality in patients with systemic inflammatory response syndrome: an evaluation of two prognostic models, two soluble receptors, and a macrophage migration inhibitory factor. Eur J Clin Microbiol Infect Dis 2008;27:375-83.

21. Allgower M, Burri C. Shock index. Dtsch Med Wochenschr 1967;92:1947-50.

22. Berger T, Green J, Horeczko T, Hagar Y, Garg N, Suarez A, et al. Shock index and early recognition of 
sepsis in the emergency department: pilot study. West J Emerg Med 2013;14:168-74.

23. Yussof SJ, Zakaria MI, Mohamed FL, Bujang MA, Lakshmanan S, Asaari AH. Value of Shock Index in prognosticating the short-term outcome of death for patients presenting with severe sepsis and septic shock in the emergency department. Med J Malaysia 2012;67:406-11.

24. Rhee KJ, Fisher CJ, Jr., Willitis NH. The Rapid Acute Physiology Score. Am J Emerg Med 1987;5:278-82.

25. Lim WS, van der Eerden MM, Laing R, Boersma WG, Karalus N, Town GI, et al. Defining community acquired pneumonia severity on presentation to hospital: an international derivation and validation study. Thorax 2003;58:377-82.

26. Subbe CP, Kruger M, Rutherford P, Gemmel L. Validation of a modified Early Warning Score in medical admissions. QJM 2001;94:521-6.

27. Geier F, Popp S, Greve Y, Achterberg A, Glockner E, Ziegler R, et al. Severity illness scoring systems for early identification and prediction of in-hospital mortality in patients with suspected sepsis presenting to the emergency department. Wien Klin Wochenschr 2013;125:508-15.

28. Cildir E, Bulut M, Akalin H, Kocabas E, Ocakoglu G, Aydin SA. Evaluation of the modified MEDS, MEWS score and Charlson comorbidity index in patients with community acquired sepsis in the emergency department. Intern Emerg Med 2013;8:255-60.

29. Fine MJ, Auble TE, Yealy DM, Hanusa BH, Weissfeld LA, Singer DE, et al. A prediction rule to identify low-risk patients with community-acquired pneumonia. N Engl J Med 1997;336:243-50.

30. Wilson AH, Kidd AC, Skinner J, Musonda P, Pai Y, Lunt CJ, et al. A simple 5-point scoring system, NaURSE ( $\mathrm{Na}+$, urea, respiratory rate and shock index in the elderly), predicts in-hospital mortality in oldest old. Age Ageing 2014;43:352-7.

31. Sivayoham N, Rhodes A, Cecconi M. The MISSED score, a new scoring system to predict Mortality In Severe Sepsis in the Emergency Department: a derivation and validation study. Eur J Emerg Med 2014;21:30-6.

32. Lemeshow S, Teres D, Klar J, Avrunin JS, Gehlbach SH, Rapoport J. Mortality Probability Models (MPM II) based on an international cohort of intensive care unit patients. JAMA 1993;270:2478-86.

33. Nguyen HB, Banta JE, Cho TW, Van Ginkel C, Burroughs K, Wittlake WA, et al. Mortality predictions using current physiologic scoring systems in patients meeting criteria for early goal-directed therapy and the severe sepsis resuscitation bundle. Shock 2008;30:23-8.

34. Marshall JC, Cook DJ, Christou NV, Bernard GR, Sprung CL, Sibbald WJ. Multiple organ dysfunction score: a reliable descriptor of a complex clinical outcome. Crit Care Med 1995;23:1638-52.

35. Knaus WA, Zimmerman JE, Wagner DP, Draper EA, Lawrence DE. APACHE-acute physiology and chronic health evaluation: a physiologically based classification system. Crit Care Med 1981;9:591-7.

36. Levy MM, Fink MP, Marshall JC, Abraham E, Angus D, Cook D, et al. 2001 SCCM/ESICM/ACCP/ATS/SIS International Sepsis Definitions Conference. Crit Care Med 2003;31:1250-6.

37. Bone RC, Sibbald WJ, Sprung CL. The ACCP-SCCM consensus conference on sepsis and organ failure. Chest 1992;101:1481-3.

38. Hilderink MJ, Roest AA, Hermans M, Keulemans YC, Stehouwer CD, Stassen PM. Predictive accuracy and feasibility of risk stratification scores for 28-day mortality of patients with sepsis in an emergency department. Eur J Emerg Med 2014 [Epub ahead of print].

39. Waldau T, Larsen VH, Bonde J. Evaluation of five oxygen delivery devices in spontaneously breathing subjects by oxygraphy. Anaesthesia 1998;53:256-63.

40. Pandharipande PP, Shintani AK, Hagerman HE, St Jacques PJ, Rice TW, Sanders NW, et al. Derivation and validation of Spo2/Fio2 ratio to impute for Pao2/Fio2 ratio in the respiratory component of the Sequential Organ Failure Assessment score. Crit Care Med 2009;37:1317-21.

41. Sakka SG, Reinhart K, Wegscheider K, Meier-Hellmann A. Variability of splanchnic blood flow in patients with sepsis. Intensive Care Med 2001;27:1281-7.

42. DeLong ER, DeLong DM, Clarke-Pearson DL. Comparing the areas under two or more correlated receiver operating characteristic curves: a nonparametric approach. Biometrics 1988;44:837-45.

43. Oddershede L, Petersen SS, Kristensen AK, Pedersen JF, Rees SE, Ehlers L. The cost-effectiveness of venous-converted acid-base and blood gas status in pulmonary medical departments. Clinico Econ Outcomes Res 2011;3:1-7. 



\section{Chapter 7}

Risk Stratification by abbMEDS and CURB-65 in relation to treatment and disposition of the septic patient at the emergency department: a cohort study

Asselina. A. Roest, Jan Tegtmeier, Joris J. Heyligen, Jeanette Duijst, Andrea Peeters, Hella F. Borgreve, Astrid M.L. Oude Lashof, Coen D.A. Stehouwer, Patricia M. Stassen BMC Emergency Medicine. 2015 Oct 13. Epub ahead of print. 


\begin{abstract}
Background

Sepsis leads to high mortality, therefore risk stratification is important. The abbMEDS (abbreviated Mortality Emergency Department Sepsis) score assesses sepsis severity and predicts mortality. In community-acquired pneumonia, the CURB-65 (Confusion, Urea, Respiration, Blood pressure, Age) also provides support in clinical decisions regarding antibiotic treatment and disposition.

We investigated the predictive value and feasibility of the abbMEDS and CURB-65 in sepsis patients at the ED and the relationship between the scores and antibiotic treatment and disposition (i.e. admission and type of ward).
\end{abstract}

\title{
Methods
}

In this retrospective cohort study, we included 725 sepsis patients at the ED. We investigated the value in predicting 28-day mortality and feasibility of both scores. We calibrated the abbMEDS. We further assessed the relationship between the three risk categories per score and antibiotic treatment (i.e. oral and intravenous narrow or broad-spectrum) and disposition.

\section{Results}

Both abbMEDS and CURB-65 were good predictors of 28-day mortality (13.0\%) (AUC $0.77[95 \% \mathrm{Cl} 0.72-0.83]$ and $0.73[95 \% \mathrm{Cl} 0.67-0.78]$ respectively) and feasible (complete score 92.7 and $93.9 \%$, respectively). In the high risk category of the abbMEDS, all patients were admitted and treated with intravenous broad-spectrum antibiotics. In the high risk category of the CURB-65, 2.5\% were not admitted and $4.4 \%$ received no antibiotics.

\section{Conclusion}

Both abbMEDS and CURB-65 are good predictors of 28-day mortality in septic ED patients. The abbMEDS is well calibrated. A new finding is that the abbMEDS matches current clinical decisions concerning antibiotic treatment and disposition, while this is less so for the CURB-65. In the future, use of the abbMEDS at the ED may improve sepsis care when its value as a decision support tool can be confirmed. 


\section{Introduction}

Sepsis, which is defined as a systemic inflammatory response syndrome (SIRS) to an infection, is a broad clinical entity and a deadly disease [1, 2]. Sepsis can be classified as sepsis, severe sepsis or septic shock, which are associated with an increasing in-hospital mortality of $16 \%$ (sepsis) to $46 \%$ (septic shock) [1,2,3]. Therefore, sepsis is an important cause of death and puts significant strain on healthcare budgets [4].

Risk stratification tools aim to assess severity of illness. In patients with communityacquired pneumonia (CAP), the CURB-65 score (Confusion, Urea, Respiration, Blood pressure, Age $>65$ years) stratifies patients into three risk categories (low, intermediate, high) that predict 28-day mortality well [5]. When applied to patients with suspected infection from all foci (including CAP), the CURB-65 had good discriminatory value with regard to 28-day in-hospital mortality [6]. Besides prediction of mortality for patients with CAP, the CURB-65 provides decision support for the physician, as it advises on two management decisions, namely choice of antibiotics (e.g. small or broad-spectrum) and disposition (i.e. whether a patient should be admitted to the hospital and to what type of ward) $[7,8]$. The CURB-65 can therefore (in patients with CAP) be used as a decision support tool in addition to the use as an estimator of mortality risk. For patients with sepsis, the abbMEDS (Abbreviated Mortality in Emergency Department Sepsis) score, derived from the MEDS (Mortality in Emergency Department Sepsis) score has good predictive value concerning 28-day mortality $[9,10]$. It would be ideal, however, if the abbMEDS could provide the same clinical guidance for sepsis as the CURB-65 does for CAP. Since there is an overlap between sepsis and CAP, we hypothesize that both the abbMEDS and CURB-65 risk categories can be related to decisions concerning management in sepsis patients.

The aim of our study was to investigate the predictive value and feasibility of the abbMEDS and CURB-65 in internal medicine patients presenting with sepsis at the ED. In addition, we aimed to assess the relationship between the three risk categories defined by both scores and two clinical decisions, i.e. empirical antibiotic treatment and disposition.

\section{Materials and methods}

\section{Study design and setting}

We performed a retrospective study of a cohort of patients. The Maastricht University Medical Centre (MUMC+) is a secondary and tertiary care university hospital in The $\mathrm{Ne}$ therlands with a capacity of 715 beds. There are 30,235 ED visits per year; approximately 5000 patients are assessed by an internist. Most patients are referred by a general practitioner before visiting the ED [11]. This study is approved by the Medical Ethics Committee of MUMC+. 


\section{Selection of participants}

We reviewed the ED charts of all patients who visited the ED between 1 April 2011 and 1 August 2012 and who were assessed by an internist. Part of these patients ( $n=704 ; 81.2 \%$ ) have been included in another study in which we investigated which sepsis patients were transported by ambulance [12]. We included patients above 18 years of age, who had two or more SIRS criteria and either suspected or proven infection (i.e. who fulfilled the criteria for sepsis, severe sepsis or septic shock) [1,3]. Patients were excluded if the current admission was a re-admission within the study period $(n=108,12.5 \%)$, or when data on 28-day mortality could not be retrieved $(n=21,2.4 \%)$, despite contacting the general practitioner. Furthermore, patients were excluded when the ED chart was not complete $(n=7,0.8 \%)$ or when the patient died at the ED before the chart was completed $(n=1,0.1 \%)$.

\section{Methods and measurements}

All data were retrieved from the ED charts and the hospital's electronic database by the use of standardized forms and by two independent researchers. We recorded date of birth, sex, comorbidity, vital signs, laboratory results, suspected focus of infection, items of the abbMEDS and CURB-65 score (Appendix, Table 6), antibiotic treatment, results of microbiology cultures, disposition (admission and type of ward), length of hospital stay and 28day mortality.

Since the respiratory rate and Glasgow Coma Scale were not always exactly documented at the ED charts, we derived these items from other information in the chart in the same way as others did [6]. Items of the abbMEDS and CURB-65 were derived from patient charts. The abbMEDS and CURB-65 score were calculated and patients were assigned to the three defined risk categories (low, intermediate, high) of each score (Appendix, Table 6$)[5,9]$. To assess the discriminatory value of both scores in the daily practice of a busy $E D$, only complete scores were included in our analysis with the exception of the missing abbMEDS item 'nursing home resident', which we imputed. This item could not always be scored $(n=113,15.6 \%)$ and we therefore made the assumption that all patients under 65 years of age ( $n=68,9.4 \%$ ) were not nursing home residents, unless stated otherwise. Suspected focus of infection was determined by the treating physician at the ED.

Antibiotic treatment was categorized into four groups: no antibiotics, oral antibiotics, intravenous (IV) narrow-spectrum, or IV broad-spectrum (Appendix, Table 7). When a patient received two narrow-spectrum antibiotics, which covered both gram positive and gram negative bacteria, we classified this as IV broad-spectrum antibiotics. Results of microbiological cultures were recorded per site (blood, urine, skin/wound and sputum). To minimize the chance of confusing infections present at the ED with nosocomial infections, we only recorded results of cultures taken at the day of presentation and two subsequent days $[13,14]$.

With regard to the adequacy of current empirical antibiotic treatment, we classified the choice of antibiotics started at the ED as adequate when the identified microorganisms were susceptible for the antibiotics in vitro. If a patient had a positive culture, but received 
no antibiotics, treatment was considered inadequate. When multiple micro-organisms were cultured, all had to be susceptible for the administered antibiotics. As coagulasenegative staphylococci in blood cultures can result from contamination, discharge letters were checked to retrieve whether the treating physician(s) regarded the culture as relevant or as contamination.

Regarding disposition, all patients were stratified into three groups: no admission, admission to regular ward and medium/intensive care unit (MCU/ICU).

\section{Outcomes}

To assess the discriminatory value of the abbMEDS and CURB-65 in predicting 28-day mortality, we plotted Receiver Operation Characteristic (ROC) curves and calculated the Area Under the Curve (AUC) with 95\% confidence intervals (CI). To calibrate the abbMEDS, we compared the observed data on 28-day mortality per risk category with those found in the derivation study of Vorwerk et al. [9]. Since the CURB-65 has only been derived in CAP patients, we did not calibrate the CURB-65 in our sepsis cohort. To assess the feasibility of the abbMEDS and CURB-65, we calculated the total number and proportion of complete scores in our study population.

We investigated two clinical decisions, i.e. the choice regarding empirical antibiotic treatment and the choice regarding disposition in relation to the three abbMEDS and CURB65 risk categories. As current empirical antibiotic treatment and disposition might not be optimal, we assessed 28-day mortality for each of the groups to check for differences in mortality. To further evaluate antibiotic treatment, we investigated its adequacy in the subset of patients with positive cultures. Again, mortality was calculated for the groups with adequate and inadequate treatment.

\section{Analysis}

We performed all statistical analyses with SPSS v.20.0 (IBM SPSS Statistics, Chicago, Illinois, USA, 2011) and MedCalc v13.3 (MedCalc Software bvba, Ostend, Belgium, 2014). Continuous data were reported as means and standard deviations or medians with interquartile ranges, categorical data as absolute counts and percentages. All percentages were valid percentages. We used the DeLong test to compare AUC values [15]. Chi-square or Fisher's exact tests were used to test for differences in proportions. The Mann Whitney test was used to compare medians, one way ANOVA was used to analyse trends. We analysed mortality between the different groups of antibiotic treatment and disposition with KaplanMeier survival analysis and the Log rank test. Associations or differences were considered statistically significant if $p<0.05$. 


\section{Results}

\section{Patients}

In the 15 month study period, 6937 patients were assessed by an internist at the ED. Of these, 862 had sepsis. We excluded 137 to form a final cohort of 725 patients (Figure 1).

In our patients, the prevalence of comorbidity was high, as $31.3 \%$ had a malignancy and $37.5 \%$ cardiopulmonary disease (Table 1). Ninety-four patients (13.0\%) died within 28 days after presentation. The median age was higher in non-survivors than in survivors (73.5 vs. 64.0 years, $\mathrm{p}<0.0001)$. The abbMEDS and CURB- 65 scores were higher in non-survivors than in survivors as well $(p<0.0001)$.

Figure 1 Flowchart of study population

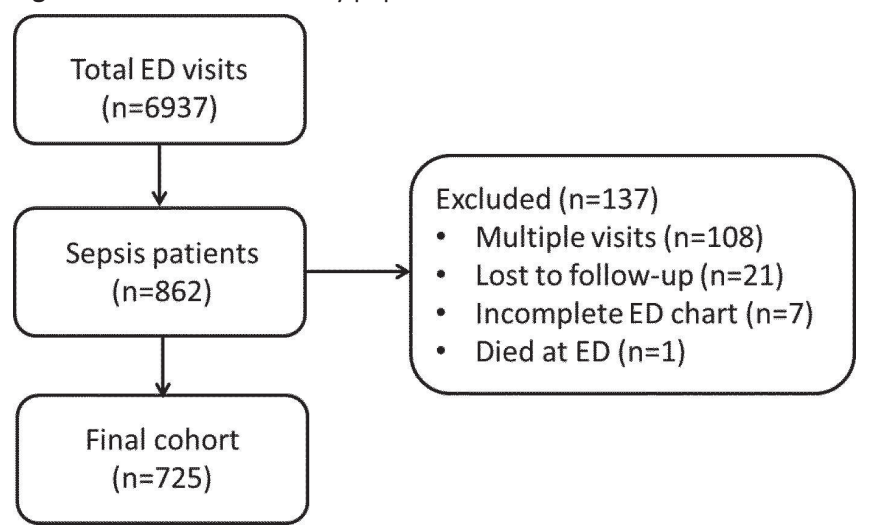


Table 1 Patient characteristics and management

\begin{tabular}{|c|c|c|c|}
\hline n (\%) or median (IQR) & $\begin{array}{l}\text { All patients } \\
\mathrm{n}=725\end{array}$ & \begin{tabular}{|l} 
Survivors \\
$n=631(87.0)$
\end{tabular} & $\begin{array}{l}\text { Non-survivors } \\
n=94(13.0)\end{array}$ \\
\hline Age, years & $65.0(53.0-77.0)$ & $64.0(51.0-76.0)$ & $73.5(63.8-83.0)^{*}$ \\
\hline Age $>65$ & $351(48.4)$ & $284(45.0)$ & $67(71.3)^{*}$ \\
\hline Male sex & $338(46.6)$ & $299(47.4)$ & $39(41.5)$ \\
\hline \multicolumn{4}{|l|}{ Comorbidity } \\
\hline Cancer & $292(40.3)$ & $247(39.1)$ & 45 (47.9) \\
\hline Cardiopulmonary & $233(32.1)$ & $189(30.0)$ & $44(46.8)^{*}$ \\
\hline Immunocompromised & $198(27.3)$ & $175(27.7)$ & $23(24.5)$ \\
\hline Neuropsychiatric & $177(24.4)$ & $149(23.6)$ & $28(29.8)$ \\
\hline Diabetes mellitus & $134(18.5)$ & $110(17.4)$ & $24(25.5)$ \\
\hline Renal disease & $96(13.2)$ & $83(13.2)$ & $13(13.8)$ \\
\hline Liver disease & $22(3.0)$ & $21(3.3)$ & $1(1.1)$ \\
\hline \multicolumn{4}{|l|}{ Suspected focus of infection } \\
\hline Lower respiratory tract & $213(29.4)$ & $179(28.4)$ & $34(36.2)$ \\
\hline Urinary tract & $152(21.0)$ & $138(21.9)$ & $14(14.9)$ \\
\hline Gastrointestinal tract & $94(13.0)$ & $82(13.0)$ & $12(12.8)$ \\
\hline Hepatobiliary system & $43(5.9)$ & $37(5.9)$ & $6(6.4)$ \\
\hline Skin & $39(5.4)$ & $34(5.4)$ & $5(5.3)$ \\
\hline Upper respiratory tract & $37(5.1)$ & $36(5.7)$ & $1(1.1)$ \\
\hline Other & $147(20.3)$ & $125(19.8)$ & $22(23.4)$ \\
\hline Antibiotics at ED & $647(89.2)$ & $558(88.4)$ & $89(94.7)$ \\
\hline \multicolumn{4}{|l|}{ Disposition } \\
\hline No admission & $63(8.7)$ & $62(9.8)$ & $1(1.1)^{\dagger}$ \\
\hline Regular ward & $626(86.3)$ & $544(86.2)$ & $82(87.2)$ \\
\hline $\mathrm{MCU} / \mathrm{ICU}$ & $36(5.0)$ & $25(4.0)$ & $11(11.7)^{\ddagger}$ \\
\hline Length of hospital stay, days & $6(3-11)$ & $7(3-12)$ & $5(2-10)^{\ddagger}$ \\
\hline \multicolumn{4}{|l|}{ Severity scores } \\
\hline AbbMEDS & $5(2-8)$ & $3(2-7)$ & $8(6-11) *$ \\
\hline CURB-65 & $1(0-2)$ & $1(0-2)$ & $2(1-3)^{*}$ \\
\hline
\end{tabular}

\section{Discriminatory value}

Mortality increased per increasing risk category for both scores (Figure 2). Compared to the abbMEDS, the CURB-65 assigned more patients to the high risk category ( 5.8 vs. $23.3 \%$ ). The AUC of the abbMEDS was 0.77 [ $95 \% \mathrm{Cl}: 0.72-0.83$ ] and that of the CURB-65 0.73 [95\% $\mathrm{Cl}: 0.67-$ 0.78 ] for predicting 28 -day mortality. This difference was not statistically significant $(p=0.08)$. 
Figure 2 Number of patients and 28-day mortality for the three abbMEDS and CURB-65 risk categories

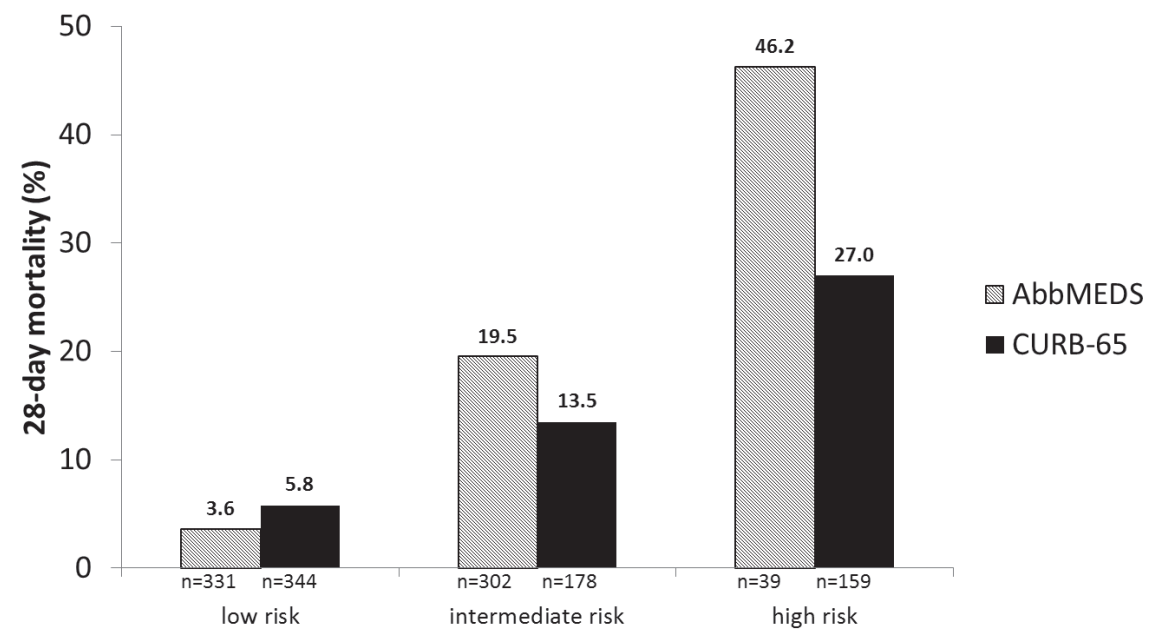

Feasibility

Complete risk scores could be calculated in 672 (92.7\%) patients for the abbMEDS, and in 681 (93.9\%) for the CURB-65 ( $p=0.51)$. The abbMEDS was incomplete due to missing information concerning the item "nursing home resident" ( $n=45,6.2 \%$ (>65 years)) or missing thrombocytes ( $n=8,1.1 \%$ ). The CURB-65 score was incomplete mostly due to missing urea values $(n=42,5.8 \%)$. The survival of those with complete and incomplete scores was not different $(p=0.42)$.

\section{Calibration}

Table 2 shows that mortality increased per increasing abbMEDS risk category in parallel with the mortality found in the derivation study by Vorwerk et al. [9]. In our study, mortality in the low risk category was higher than in the derivation study (3.6 vs. 1.6\%), while in the intermediate and high risk categories our mortality was lower (19.5 vs. $23.4 \%$ and 46.2 vs. 59.0\%, respectively).

Table 2 Mortality within 28 days per abbMEDS risk category compared to the derivation study (10)

\begin{tabular}{|l|r|r|r|r|}
\hline Risk category & \multicolumn{4}{|c|}{ 28-day mortality, $\mathbf{n}$ (\%) } \\
\hline & \multicolumn{2}{|c|}{ Current study } & \multicolumn{2}{c|}{ Derivation study (10) } \\
\hline & Mortality & \multicolumn{1}{|c|}{ Patients } & \multicolumn{1}{c|}{ Mortality } & \multicolumn{1}{c|}{ Patients } \\
\hline Low risk & $12(3.6)$ & 331 & $1(1.6)$ & 63 \\
\hline Intermediate risk & $59(19.5)$ & 302 & $48(23.4)$ & 205 \\
\hline High risk & $18(46.2)$ & 39 & $23(59.0)$ & 39 \\
\hline Total & $89(13.2)$ & 672 & $72(23.5)$ & 307 \\
\hline
\end{tabular}




\section{Antibiotic treatment in relation to risk categories}

Of the 725 patients, $78(10.8 \%)$ did not receive antibiotic treatment, 71 (9.8\%) received oral and $34(4.7 \%)$ IV narrow-spectrum antibiotics and the majority were treated with IV broad-spectrum antibiotics (74.7\%, Table 3 ). In the low and intermediate risk categories of both scores, antibiotic treatment was almost similar. In contrast, all high risk abbMEDS patients were treated with IV broad-spectrum antibiotics, while 15 (9.4\%) high risk CURB65 patients were treated with no, oral or IV narrow-spectrum antibiotics. There were no significant differences in mortality between the four groups of antibiotic treatment in the intermediate risk category of both scores, but the numbers were too small to draw firm conclusions. Mortality in the four groups of antibiotic treatment in the low and high risk categories were not compared with each other, because in some subgroups nobody died.

Table 3 Antibiotic treatment and 28-day mortality per risk category of the abbMEDS and CURB-65

\begin{tabular}{|c|c|c|c|c|c|c|}
\hline \multirow[t]{2}{*}{$n(\%)$} & \multicolumn{2}{|c|}{ Low risk } & \multicolumn{2}{|c|}{ Intermediate risk } & \multicolumn{2}{|c|}{ High risk } \\
\hline & Patients & Mortality & Patients & Mortality & Patients & Mortality \\
\hline \multicolumn{7}{|l|}{ AbbMEDS } \\
\hline No antibiotics & $52(15.7)$ & $1(1.9)$ & $20(6.6)$ & $4(20.0)$ & 0 & 0 \\
\hline Oral & $41(12.4)$ & 0 & $21(7.0)$ & $3(14.3)$ & 0 & 0 \\
\hline $\begin{array}{l}\text { IV narrow- } \\
\text { spectrum }\end{array}$ & $22(6.6)$ & 0 & $10(3.3)$ & $1(10.0)$ & 0 & 0 \\
\hline $\begin{array}{l}\text { IV broad- } \\
\text { spectrum }\end{array}$ & $216(65.3)$ & $11(5.1)$ & $251(83.1)$ & $51(20.3)$ & $39(100.0)$ & $18(46.2)$ \\
\hline Total $(672)^{\#}$ & $331(49.3)$ & $12(3.6)$ & 302 (44.9) & $59(19.5)$ & $39(5.8)$ & $18(46.2)$ \\
\hline \multicolumn{7}{|l|}{ CURB-65 } \\
\hline No antibiotics & $54(15.7)$ & $3(5.6)$ & $10(5.6)$ & 0 & $7(4.4)$ & $1(14.3)$ \\
\hline Oral & $47(13.7)$ & $3(6.4)$ & $13(7.3)$ & $1(7.7)$ & $4(2.5)$ & 0 \\
\hline $\begin{array}{l}\text { IV narrow- } \\
\text { spectrum }\end{array}$ & $24(7.0)$ & 0 & $5(2.8)$ & 0 & $4(2.5)$ & 0 \\
\hline $\begin{array}{l}\text { IV broad- } \\
\text { spectrum }\end{array}$ & $219(63.7)$ & $14(6.4)$ & $150(84.3)$ & $23(15.3)$ & $144(90.6)$ & $41(28.5)$ \\
\hline Total (681)\# & $344(50.5)$ & $20(5.8)$ & $178(26.1)$ & $24(13.5)$ & $159(23.3)$ & 42 (26.4) \\
\hline
\end{tabular}

\section{Adequacy of empirical antibiotic treatment in relation to risk categories}

One or more positive culture(s) were found in 244 patients (Appendix, Table 8). Seventeen patients were excluded because their cultures were considered to result from contamination. Empirical antibiotic treatment was considered adequate in 169 (74.4\%) of the remaining 227 patients (Table 4). There was no significant difference in 28-day mortality between the groups with adequate $(13.6 \%)$ and inadequate treatment $(12.1 \%, p=0.48, \log$ 
rank test). The higher the abbMEDS risk category, the more frequent antibiotic treatment was considered adequate $(p=0.04)$. For the CURB-65, adequacy of antibiotic treatment was not different between the three risk categories $(p=0.09)$.

Table 4 Adequacy of antibiotic treatment and 28-day mortality per risk category of the abbMEDS and CURB-65

\begin{tabular}{|c|c|c|c|c|c|c|}
\hline \multirow[t]{2}{*}{ n (\%) } & \multicolumn{2}{|c|}{ Low risk } & \multicolumn{2}{|c|}{ Intermediate risk } & \multicolumn{2}{|c|}{ High risk } \\
\hline & Patients & Mortality & Patients & Mortality & Patients & Mortality \\
\hline \multicolumn{7}{|l|}{ AbbMEDS } \\
\hline Adequate* & $60(65.9)$ & $1(1.7)$ & $80(80.8)$ & $13(16.3)$ & $13(92.9)$ & $6(46.2)$ \\
\hline Inadequate & $31(34.1)$ & $1(3.2)$ & $19(19.2)$ & $5(26.3)$ & $1(7.1)$ & $1(100)$ \\
\hline Total $(204)^{\#}$ & 91 & $2(2.2)$ & 99 & $18(18.2)$ & 14 & $7(50.0)$ \\
\hline \multicolumn{7}{|l|}{ CURB-65 } \\
\hline Adequate & $61(70.1)$ & $4(6.6)$ & $43(70.5)$ & $3(7.0)$ & $58(84.1)$ & $15(25.9)$ \\
\hline Inadequate & $26(29.9)$ & $1(3.8)$ & $18(29.5)$ & $5(27.8)$ & $11(15.9)$ & $1(9.1)$ \\
\hline Total $(217)^{\#}$ & 87 & $5(5.7)$ & 61 & $8(13.1)$ & 69 & $16(23.2)$ \\
\hline
\end{tabular}

\section{Disposition in relation to risk categories}

Across the three risk categories of both scores, the majority of patients were admitted to the hospital (Table 5). The main difference between both scores was that all high risk abbMEDS patients were admitted, while four (2.5\%) high risk CURB-65 patients were not admitted. The proportion of patients, who were treated as outpatients, was highest in the low risk category and almost equal for both the abbMEDS and CURB-65. The only patient in the intermediate risk category of the abbMEDS who was treated as an outpatient, had terminal cancer and went home on his own request, where he died. None of the CURB65 patients, treated as outpatients, died. While most patients were admitted to a regular ward, the percentage of patients admitted to a MCU/ICU increased through the risk categories in both scores to 12.6 and $11.9 \%$ in the high risk category of abbMEDS and CURB-65, respectively. 
Table 5 Disposition and 28-day mortality per risk category of the abbMEDS and CURB-65

\begin{tabular}{|l|r|r|r|r|r|r|}
\hline $\mathbf{n}$ (\%) & \multicolumn{2}{|c|}{ Low risk } & Intermediate risk & \multicolumn{1}{c|}{ High risk } \\
\hline & Patients & Mortality & Patients & Mortality & Patients & Mortality \\
\hline AbbMEDS \\
\hline No admission & $40(12.1)$ & 0 & $16(5.3)$ & $1(6.3)$ & 0 & 0 \\
\hline Regular ward & $289(87.3)$ & $12(4.2)$ & $259(85.8)$ & $50(19.3)$ & $34(87.2)$ & $15(44.1)$ \\
\hline MCU/ICU & $2(0.6)$ & 0 & $27(8.9)$ & $8(29.6)$ & $5(12.8)$ & $3(60.0)$ \\
\hline Total (672) & 331 & $12(3.6)$ & 302 & $59(19.5)$ & 39 & $18(46.2)$ \\
\hline CURB-65 & \multicolumn{7}{|c|}{0} \\
\hline No admission & $46(13.4)$ & 0 & $5(2.8)$ & 0 & $4(2.5)$ & 0 \\
\hline Regular ward & $295(85.8)$ & $19(6.4)$ & $161(90.4)$ & $20(12.4)$ & $136(85.5)$ & $38(27.9)$ \\
\hline MCU/ICU & $3(0.9)$ & 1 (33.3) & $12(6.7)$ & $4(33.3)$ & $19(11.9)$ & $5(26.3)$ \\
\hline Total (681) & 344 & $20(5.8)$ & 178 & $24(13.5)$ & 159 & $43(27.0)$ \\
\hline $\begin{array}{l}\text { There were no significant differences in mortality per disposition group within each risk category. } \\
\text { \# The totals are depicted to show that not for all analyses, data for all patients were available. }\end{array}$ \\
\hline
\end{tabular}

\section{Discussion}

This study investigated the value of abbMEDS and CURB-65 in internal medicine patients presenting with sepsis at the ED. We found a moderate predictive value regarding 28-day mortality of both the abbMEDS (AUC 0.77) and CURB-65 (AUC 0.73). In addition, both scores were feasible at our ED with complete scores in 92.7 and $93.9 \%$, respectively. New findings are that the abbMEDS categorizes patients well with respect to two important clinical decisions, namely empirical antibiotic treatment (e.g. narrow or broad-spectrum) and disposition (i.e. whether a patient is admitted to the hospital and to what level of care), and that the abbMEDS performs better than the CURB- 65 .

With regard to the discriminatory value of the AbbMEDS, we found a slightly lower AUC than in the derivation study (AUC 0.77 vs. 0.82 ) by Vorwerk et al. [9], which may result from a difference in patient selection. The abbMEDS was well calibrated in our population, but compared to the derivation study, we found a higher mortality in the low risk category (3.6 vs. 1.6\%). An explanation for this finding may be that, due to the prominent role of the general practitioner in the Netherlands, the patients who are referred to the hospital and then assigned to the low risk category are sicker than patients in other countries $[11,16]$. The CURB-65 was developed for patients with CAP [5]. Later, the CURB-65 was validated in an ED population with an infection [6]. In that study, a higher AUC (0.79) was found than in ours (0.73), which might be explained by the higher mortality in our study population (13.0 vs. $3.9 \%)$. The mortality in our study was higher because we selected patients with sepsis instead of patients with infection. 
The first new finding of our study is that the abbMEDS matches two current clinical decisions in the management of sepsis patients, namely empirical antibiotic treatment and disposition. This is less the case for the CURB-65. In the low risk categories of both scores, roughly $30 \%$ did not receive antibiotics or were treated with oral antibiotics (i.e. treatment that would not require admission to the hospital). The most severely ill patients in the high risk categories received almost exclusively IV broad-spectrum antibiotics. This is where the abbMEDS matches current clinical decisions better than the CURB-65, for all high risk abbMEDS patients were treated with IV broad-spectrum antibiotics, in contrast to $9.4 \%$ of the high risk CURB-65 patients who were treated with no or oral antibiotics. We did not find significant differences in mortality between these different treatment groups. However, our mortality figures are too small to draw conclusions on this matter. We additionally analysed adequacy of our empirical treatment (antibiotics started at the ED) based on in vitro susceptibility. We found that adequacy was related to the abbMEDS risk categories. With increasing risk category of the abbMEDS, more patients were treated with adequate antibiotics. However, this was not the case for CURB-65, as $15.9 \%$ of the patients in the high risk category received inadequate treatment. Again, mortality figures were too small to draw conclusions. The need for high adequacy is most urgent in the high risk category with the most severely ill patients [17]. For the less severely ill patients, a lower adequacy can be accepted, as confining microbial resistance to antibiotics is more important in this large group of patients.

The second new finding regarding clinical decisions was that the abbMEDS matches current management regarding disposition. Again, the CURB-65 did this less well. With increasing risk category of the abbMEDS, more patients were admitted to the MCU/ICU, while the fraction of patients who were not admitted to the hospital decreased. In contrast, in the high risk category of the CURB-65, 4 (2.5\%) patients were not admitted.

To our knowledge, we showed for the first time that the abbMEDS matches current clinical decisions regarding empirical antibiotic treatment and disposition of sepsis patients at the ED. In patients with CAP, the CURB-65 is already used to provide clinical decision support. This resulted in more standardized care and reduced the use of broad-spectrum antibiotics, while patient safety was maintained $[18,19]$. We think that the abbMEDS has the potential to provide clinical decision support in patients presenting with sepsis. However, future studies are needed and these should confirm that the abbMEDS can indeed appropriately and safely be used to provide this support on clinical decisions. The abbMEDS could then develop from a risk stratification score into a clinical decision support tool with the potential to increase the standard of care in sepsis patients. Hopefully, this will yield improved survival, reduced use of broad-spectrum antibiotics and reduced healthcare costs in sepsis patients.

This study has some limitations. First, we cannot exclude the risk of misclassification of measurements or data. However, we used standardized extraction forms and the main factors studied (laboratory values, antibiotic treatment, disposition and mortality) are less likely to be subject of misclassification. Second, our study was limited to one hospital, but we included over 700 patients in a 15-month study period. Third, current clinical decisions 
might not be optimal as they are physician-dependent. However, our study reflects clinical practice and it is improbable that our management is less optimal than in other hospitals. A way to examine adequate management in a better way is to ask a panel of experts whether they agree with the management decisions based on the calculated score in selected cases. Considering the limitations, our study must be seen as an exploratory one. To our opinion, the results justify a prospective study that implements such a panel.

\section{Conclusion}

Both the abbMEDS and CURB-65 are good predictors of 28-day mortality in sepsis patients and are very feasible at the ED. The abbMEDS is well calibrated. A new finding is that the abbMEDS matches current clinical decisions in sepsis patients concerning antibiotic treatment and disposition well, while this is less the case for the CURB-65. To improve the standard of care of sepsis, further research on the ability of the abbMEDS to provide support in clinical decisions in sepsis patients at the ED must be performed.

\section{Acknowledgements}

G. Vandergraesen, staff supporter of the emergency department MUMC+ 


\section{References}

1. Bone RC, Sibbald WJ, Sprung CL. The ACCP-SCCM consensus conference on sepsis and organ failure. Chest. 1992;101:1481-3.

2. Rangel-Frausto MS, Pittet D, Costigan M, Hwang T, Davis CS, Wenzel RP. The natural history of the systemic inflammatory response syndrome (SIRS). A prospective study. JAMA. 1995;273:117-23.

3. Levy MM, Fink MP, Marshall JC, Abraham E, Angus D, Cook D, et al. 2001 SCCM/ESICM/ACCP/ATS/SIS International Sepsis Definitions Conference. Crit Care Med. 2003;31:1250-6.

4. Bakker J, Levi M, van Hout BA, van Gestel A. Sepsis, a complicated syndrome with major medical and social consequences. Ned Tijdschr Geneeskd. 2004;148:975-8.

5. Lim WS, van der Eerden MM, Laing R, Boersma WG, Karalus N, Town GI, et al. Defining community acquired pneumonia severity on presentation to hospital: an international derivation and validation study. Thorax. 2003;58:377-82.

6. Howell MD, Donnino MW, Talmor D, Clardy P, Ngo L, Shapiro NI. Performance of severity of illness scoring systems in emergency department patients with infection. Acad Emerg Med. 2007;14:709-14.

7. Harris M, Clark J, Coote N, Fletcher P, Harnden A, McKean M, et al. British Thoracic Society guidelines for the management of community acquired pneumonia in children: update 2011. Thorax. 2011;66 Suppl 2:ii1-23.

8. Wiersinga WJ, Bonten MJ, Boersma WG, Jonkers RE, Aleva RM, Kullberg BJ, et al. SWAB/NVALT (Dutch Working Party on Antibiotic Policy and Dutch Association of Chest Physicians) guidelines on the management of community-acquired pneumonia in adults. NethJ Med. 2012;70:90-101.

9. Vorwerk C, Loryman B, Coats TJ, Stephenson JA, Gray LD, Reddy G, et al. Prediction of mortality in adult emergency department patients with sepsis. Emerg Med J. 2009;26:254-8.

10. Shapiro NI, Wolfe RE, Moore RB, Smith E, Burdick E, Bates DW. Mortality in Emergency Department Sepsis (MEDS) score: a prospectively derived and validated clinical prediction rule. Crit Care Med. 2003;31:670-5.

11. Thijssen WA, Giesen PH, Wensing M. Emergency departments in The Netherlands. Emerg Med J. 2012;29:6-9.

12. Groenewoudt M, Roest AA, Leijten FM, Stassen PM. Septic patients arriving with emergency medical services: a seriously ill population. Eur J Emerg Med. 2014;21(5):330-5.

13. Artero A, Zaragoza R, Camarena JJ, Sancho S, Gonzalez R, Nogueira JM. Prognostic factors of mortality in patients with community-acquired bloodstream infection with severe sepsis and septic shock. J Crit Care. 2010;25:276-81.

14. Grozdanovski K, Milenkovic Z, Demiri I, Spasovska K. Prediction of outcome from community-acquired severe sepsis and septic shock in tertiary-care university hospital in a developing country. Crit Care Res Pract. 2012;2012:182324.

15. DeLong ER, DeLong DM, Clarke-Pearson DL. Comparing the areas under two or more correlated receiver operating characteristic curves: a nonparametric approach. Biometrics. 1988;44:837-45.

16. Hermans MA, Leffers P, Jansen LM, Keulemans YC, Stassen PM. The value of the Mortality in Emergency Department Sepsis (MEDS) score, C reactive protein and lactate in predicting 28-day mortality of sepsis in a Dutch emergency department. Emerg Med J. 2012;29:295-300.

17. Chen HC, Lin WL, Lin CC, Hsieh WH, Hsieh CH, Wu MH, et al. Outcome of inadequate empirical antibiotic therapy in emergency department patients with community-onset bloodstream infections. J Antimicrob Chemother. 2013;68:947-53.

18. Chalmers JD, Singanayagam A, Akram AR, Choudhury G, Mandal P, Hill AT. Safety and efficacy of CURB65-guided antibiotic therapy in community-acquired pneumonia. J Antimicrob Chemother. 2011;66:416-23.

19. Singanayagam A, Chalmers JD. Severity assessment scores to guide empirical use of antibiotics in community acquired pneumonia. Lancet Resp Med. 2013;1:653-62. 


\section{Chapter 8}

General discussion 
The studies in this thesis describe the journey through the chain of emergency care from diagnosing sepsis (chapter 2) to prehospital care in sepsis patients (chapters 3 and 4 ) and risk stratification of sepsis patients at the Emergency Department (ED) (chapters 5, 6 and 7). In this discussion, existing knowledge and the way our findings contribute to the improvement of emergency care in sepsis patients will be discussed. Furthermore, future perspectives of research in this field will be discussed.

\section{The definition of sepsis}

Sepsis is a serious disease leading to high morbidity and mortality, especially when accompanied by organ failure [1,2]. Early recognition of sepsis facilitates adequate treatment at an early stage [3], which ultimately reduces mortality in patients with severe sepsis and septic shock $[4,5]$. In order to intervene at an early stage, highly sensitive and specific diagnostic criteria are needed to recognize sepsis patients. The current sepsis definition is a set of diagnostic criteria: two or more SIRS-criteria and a suspected or proven infection and is designed to be highly sensitive, even at the expense of specificity [6-9], to avoid missing the serious diagnosis sepsis. Low specificity results in overtreatment with an increase in bacterial resistance, but more importantly, it could lead to missing other life-threatening diagnoses, for example a pulmonary embolism. The low specificity of the sepsis definition leads to ongoing international discussion about the usefulness of the definition in daily practice and in research.

Despite the discussions about the importance of a definition of sepsis with good diagnostic accuracy, until now, this definition has not been changed during the most recent International Sepsis Definitions Conference of 2001, nor been validated extensively [10]. In addition, the few studies that have been performed focus on intensive care patients [11-13]. Therefore, most available information concerns a relatively small number of patients who are more severely ill than the general population presenting at the Emergency Department (ED) $[14,15]$. It is not unlikely that the diagnostic value of the sepsis definition in ED patients differs from ICU patients [15]. In chapter 2, we evaluated the diagnostic value of the sepsis definition in a general medical population $(n=538)$ with and without infection at the ED. We found that the sepsis definition had a sensitivity of $73.5 \%$ and a specificity of $86.8 \%$, which means that over a quarter of the patients with sepsis were missed and almost $15 \%$ of the patients were wrongly diagnosed as having sepsis by the sepsis definition. The sensitivity of the sepsis definition at the ED was indeed lower than that reported in ICU studies [16-18]. Fortunately, the diagnosis made by the physician (face diagnosis) at the ED had higher diagnostic value than the sepsis definition; i.e. the ED physician corrected some of the flaws of the sepsis definition, probably by combining the sepsis definition with pattern recognition and by profiting from the fact that during stay at the ED, more information on the status of the patient becomes available. We were indeed able to demonstrate that during stay at the ED and in the hospital, the diagnosis is changed in $19.3 \%$ of the patients. This means that the diagnostic value of the sepsis definition is less than we first calculated, due to cross-over between the groups. 
We were not able to identify other factors (age, Charlson Comorbidity Index, vital signs or laboratory values) that could influence the discordance between the sepsis definition and the discharge diagnosis. It is possible that other factors like the experience of the physician or obtaining more detailed information on the clinical presentation and history of the patient contributed to the face and final discharge diagnosis.

In conclusion, the diagnosis made by the physician is extremely important, because decisions on treatment and disposition have to be made, based on preliminary clinical assessments and test results. This is confirmed by our finding that the combination 'sepsis by sepsis definition and/or face diagnosis' resulted in a higher diagnostic value than when the sepsis definition was used solely. This finding is in concordance with the decision to add a list of signs and symptoms of sepsis for pattern recognition in daily clinical practice to the sepsis definition during the International Sepsis Definitions Conference of 2001 [10]. However, this expanded list is not practical to use in research nor in daily practice, because it consists of more than twenty items of which some are very aspecific (hyperglycaemia, significant oedema) or not applicable at the ED (cardiac index, SvO2).

There are some limitations in chapter 2 that need to be addressed. First, we assessed the patients' charts retrospectively. This increases the chance of selection and misclassification bias. However, our charts are rather complete and include information from doctors, nurses, ancillary investigations and data from the municipal administration (regarding mortality) as well. In case of missing SIRS criteria, we deduced these from the description of the patient at the moment of presentation at the ED. Missing SIRS criteria is to be expected in patients with for example an intoxication. In such a case, it is not mandatory to measure a white blood cell count. Second, by retrieving a clinical diagnosis retrospectively, the face diagnosis and discharge diagnosis could be biased by knowledge on the presence of SIRS criteria and/or clinical outcome. We tried to minimize this problem by assessing the face diagnosis and discharge diagnosis by two investigators separately. A fourth issue might be that we considered the discharge diagnosis the "reference test" for the diagnosis of sepsis, because at discharge, most results of laboratory, cultures and radiologic examinations are known. We are aware of the fact that the discharge diagnosis depends on the ability of the hospital physician to diagnose sepsis. Another method to establish this diagnosis would be to implement a panel of experts that decides whether or not the patient has sepsis during stay in the hospital.

In the future, it may be possible to improve diagnosing of sepsis by early confirmation of infection. A confirmation of infection helps the physician to diagnose sepsis. However, it may take several days before blood, sputum or urinary cultures confirm a bacterial infection. Recently, two new methods have been developed to identify the bacterial pathogen in blood cultures in an early stage: matrix-assisted laser-desorption/ionization time-of-flight mass spectrometry (MALDI-TOF MS) and Polymerase Chain Reaction/Electrospray lonization Mass Spectometry (PCR/ESI-MS) $[19,20]$. MALDI-TOF MS can be used after an incubation period of 4 hours of the blood culture on a chocolate agar plate. It has an overall concordance of $69.5 \%$ with standard techniques, which increases to $97.6 \%$ in Gram negative 
bacteria and 100\% in Staphylococcus aureus and Enterococci [21]. PCR/ESI-MS is a newer and more expensive technique than MALDI-TOF MS. The concordance of the PCR/ESI-MS with standard techniques is very high for determining both the genus and species of the bacterial pathogen (98.7 and $96.6 \%$, respectively). In addition, in contrast to MALDI-TOF MS, PCR/ESI-MS can detect polymicrobial infections and antibiotic resistance genes [19, 20]. Unfortunately, these techniques can only be used when blood cultures are positive, which is the case in one third of the sepsis patients [22]. To diagnose the other two third of the patients another tool is still needed.

Another promising field of research in sepsis patients are biomarkers. One of the biomarkers evaluated in sepsis patients is C-reactive protein (CRP), which is an acute phase inflammation protein synthesized by the liver [23, 24]. However, CRP, like the sepsis definition, is highly sensitive to detect an inflammatory response, but lacks specificity in the detection of sepsis [24]. Procalcitonin (PCT) is normally synthesized by thyroid cells and is a pro-hormone of calcitonin, but pro-inflammatory stimuli increases the ability of other tissues and immune cells to produce PCT as well $[25,26]$. PCT correlates better with the presence of an infection than CRP, but it also rises transiently in SIRS without infection $[27,28]$. Recently, some studies present presepsin as a promising biomarker in sepsis patients [29]. Presepsin is present in the human body in low concentrations, is expressed on the surface of monocytes and macrophages and increases rapidly in response to bacterial infections [30, 31]. However, recent studies have shown conflicting results in sepsis patients [32, 33]. An explanation could be that the concentration of presepsin in specific populations is unknown. For example, concentrations of presepsin increase with progression of kidney dysfunction and age in the absence of infection [34]. There are several other biomarkers with a potential diagnostic value in sepsis patients like adrenomedullin (ADM), pro-ADM, atrial natriuretic peptide (ANP) and pro-ANP, pro-vasopressin, interferon- $\gamma$ and several interleukins [35]. However, the studies are still small, little is known about the optimal assays, adapted thresholds in specific populations and cost-effectiveness.

In conclusion, there are several biomarkers in sepsis, but until now none of them appears to be a gold standard in diagnosing sepsis. This is to be expected as the sepsis response is very complex, it is improbable that a single biomarker will be sufficient to diagnose sepsis. Most likely, in the future, the diagnosis of sepsis will be made by finding a combination of vital signs, simple laboratory results and one or more biomarkers. Therefore, prospective, multicentre studies performed in a general ED population are necessary to identify the best and fastest way to diagnose sepsis.

Meanwhile, the clinical judgment of the physician at the ED and therefore education and training of these physicians - remains very important in diagnosing sepsis.

In this thesis, the abovementioned and internationally discussed definition of sepsis is used to select our patients. However, we found in chapter 2 that the face diagnosis of sepsis performs better than the sepsis definition. Unfortunately, the face diagnosis is a black box and therefore not applicable to research. It is probable that our physicians are more experienced in diagnosing sepsis, because they are (proportionally) more often exposed to sepsis than doctors in other countries as about a quarter of the patients presenting at our ED fulfil the sepsis definition $[15,36,37]$. There are two reasons for this high exposure: 
first, most patients at our ED have been referred by a general practitioner to the internist and are therefore more severely ill $[15,36,37]$ and second, this study was performed in a secondary and tertiary teaching hospital, with a substantial number of (immune compromised) septic patients and more physicians per patient were involved. This high exposure to sepsis patients could be beneficial to the recognition of sepsis at the ED (face diagnosis) and may have led to a relatively lower diagnostic value of the sepsis definition. Therefore, especially for inexperienced physicians, with an AUC of 0.80 , the sepsis definition can help to diagnose sepsis. Further, by adjustment of the SIRS criteria to for example $\geq 3$, the most severely ill sepsis patients will be selected, which is not a reflection of an ED population and the results would not be implementable in daily practice. Also, almost all studies uses the current sepsis definition and are therefore, easy to compare with each other. In conclusion, the current sepsis definition is still used in research because, there is no other extensively tested and superior definition of sepsis.

\section{Prehospital care in sepsis patients}

Treatment of severely ill patients is often initiated by ambulance staff, for example in acute myocardial infarction or stroke [36, 37]. For sepsis as well, treatment of sepsis is most effective when started early [5]. Therefore, in theory, ambulance staff could initiate early treatment of sepsis and possibly reduce morbidity and mortality in sepsis patients. However, treatment of sepsis patients in the ambulance is only profitable when the following three conditions are met. First, sepsis patients must be recognized by ambulance staff. Second, a large proportion of sepsis patients, preferably the most severely ill, should be transported by the ambulance. Third, early treatment in the ambulance should decrease both morbidity and mortality.

First, before sepsis management can be initiated the patient should be recognized as having sepsis. Unfortunately, sepsis is a frequently missed diagnosis. Hospital doctors in Brazil did not recognize severe sepsis in $43 \%$ and in the United Kingdom, only $17 \%$ of the admitted patients with sepsis were documented to have sepsis $[38,39]$. In theory, the sepsis definition is easy to use in the pre-hospital phase as it includes items that can be measured in the ambulance (temperature, heart rate, respiratory rate), and thus recognition could be possible in most patients with sepsis. However, in two American studies, the ambulance staff missed up to $69 \%$ of those with a serious infection and $52.2 \%$ of patients with severe sepsis $[40,41]$. We investigated recognition of sepsis patients by ambulance personnel in the Netherlands (chapters 3 and 4) and found that sepsis was documented in about $60 \%$ of the ambulance charts of sepsis patients. Because both our studies were retrospective, we (like other investigators) were only able to retrieve the cases in which sepsis was documented in the chart $[3,42]$.

It seems very important to document sepsis, because patients with documented sepsis had significant lower mortality than those with non-documented sepsis ( 12.9 vs. $25.7 \%$ ). This is in agreement with another study where mortality was twice as low in ambulance patients with recognized severe sepsis than in those who were not recognized (13.5 vs. $33.3 \%$ ) [41]. We could not explain this difference in mortality by differences in age, 
comorbidity, the use of cytotoxic or immune suppressive medication, sepsis severity, initiated treatment in the ambulance and start of antibiotic treatment at the ED. However, non-documented patients stayed half an hour longer at the ED, which may indicate that treatment at the ED was started later. In other studies, patients who were recognized as septic by the paramedic received antibiotics 53 minutes earlier at the ED than those who were not recognized [43]. In conclusion, documentation of sepsis in the ambulance is associated with lower mortality, probably by improving quality of emergency care in sepsis patients at the ED, although the mechanism and factors of influence are not yet entirely clear.

To improve recognition, it is important to understand why sepsis is not recognized. We found five reasons for non-documentation of sepsis. First, lack of assessment of vital signs in the ambulance (32.1\%). Measuring vital signs contributed to the recognition of sepsis; in particular, the assessment of the temperature contributed to the documentation of sepsis (OR 11.2). Second, sepsis was not yet present in the ambulance (5.7\%), third, laboratory values ( $\mathrm{pCO} 2$, leucocytes and bands) were not measurable in the ambulance (46.4\%). Fourth, the diagnosis was missed despite having accurate information (15.7\%). Last, urgent transport was negatively associated with documentation, which may be explained by the fact that the ambulance nurse needs time to stabilize the patient and has less time to document sepsis when a patient is unstable.

These reasons for not recognizing sepsis are in accordance with an American study [41]. However, the organisation of emergency care in the United States differs from that in the Netherlands [44]. The ambulance team in the Netherlands consists of a trained driver and a highly trained nurse instead of a paramedic. In addition, as the Netherlands is a densely populated country, the ambulance can transport (almost) all urgent patients to an ED within 45 minutes and non-urgent patients within 60 minutes. A recent Dutch study reported a lower rate of documented sepsis (13.7\%) than the $60 \%$ we found [45]. An explanation could be that in our study in $20.4 \%$ of patients, all SIRS assessments were done compared to a complete set of assessments of $7.6 \%$ in their study. In addition, we considered sepsis documented when infection, fever or sepsis was documented in the chart, whereas they considered sepsis documented when the terms sepsis, severe sepsis or septic shock were documented.

In conclusion, about $60 \%$ of the ambulance patients with sepsis are currently recognized as septic, which can be improved to almost $80 \%$ when all vital signs are assessed and knowledge on sepsis is improved.

In the future, to improve recognition, measurement of all vital signs and training of ambulance personnel is important. Recently, a large trial was started in the Netherlands to investigate among other things the effect of training of ambulance personnel on recognition of sepsis [46]. Also, point of care testing (POCT) of lactate in the ambulance may improve early detection and treatment of sepsis in the ambulance. Elevated blood lactate levels are a sensitive marker of impaired perfusion and oxygenation of tissues in patients with suspected sepsis, and particularly patients with elevated blood lactate levels are most likely to benefit of early goal directed therapy (EGDT) [47]. A study that assessed the diagnostic value of a prehospital Sepsis Alert Protocol in combination with POCT of lactate showed that measurement of lactate might have assisted in identifying an additional $11.9 \%$ of am- 
bulance patients with severe sepsis [41]. The combination of training and POCT of lactate could improve early recognition of sepsis.

The ambulance may play an important role in emergency care of sepsis patients when they first, recognize them and second, transport a significant number of sepsis patients to the hospital. We found that the ambulance indeed transports a significant number of patients (chapter 3 and 4). Almost half of all sepsis patients presenting at the ED were indeed transported by ambulance, which is in agreement with other studies [45].

We analysed medical patients only and therefore, we cannot extrapolate our findings to the total number of ambulance patients with sepsis. However, we assume that the majority of sepsis patients are presented to the internist at the ED. The ambulance patients were, compared to patients transported otherwise: older, they more often had severe sepsis or septic shock and died more frequently within 28 days (17.9 vs. $7.2 \%$, chapter 4$)$. The high mortality rate of sepsis we found was also seen in studies from the United States [48].

Therefore, as the ambulance transports a substantial part and the most severely ill sepsis patients who are treated by internists at the ED, it is likely that the ambulance may indeed play an important role in the improvement of emergency care for sepsis patients. Particularly, as it is likely that the most severely ill patients benefit most by early start of treatment.

The ambulance could recognize a large number of sepsis patients. After that early treatment of sepsis becomes important, because the severity of sepsis tends to increase rapidly. More than $20 \%$ of patients with uncomplicated sepsis develop severe sepsis or septic shock within 72 hours [49]. Furthermore, each hour of delay in the administration of antibiotics at the ED increased mortality with $7.6 \%$ in patients with severe sepsis [5].

We found that our sepsis patients were not treated aggressively in the ambulance, as only $15.7 \%$ of the patients were treated with both oxygen and intravenous fluids and almost half of the patients received neither of these treatments, which was found in other studies as well (chapter 3 ) [43, 48]. Treatment in documented patients was not different from non-documented patients. In other studies, patients who were treated with intravenous fluids in the ambulance (irrespective of the presence of shock), received twice the volume of fluids in the first hour of ED stay and had a better outcome (less intubations and lower mortality) $[48,50]$. The reason that only a small number of our patients were treated in the ambulance can at least in part be explained by the current ambulance protocol, which only prescribes oxygen and intravenous fluids in case of septic shock [51]. This explanation is in line with the finding that treatment was more frequently initiated with increasing sepsis severity.

There are some limitations in chapter 3 and 4 that need to be addressed. First, recognition of sepsis in our studies was based on the documentation of sepsis in the chart. Therefore, a prospective study that focuses on the recognition of sepsis would be preferable. However, performing a prospective study to assess recognition of sepsis could also cause selection bias as the study alerts health care professionals, thereby improving the results. A second retrospective study is an option when registration of a diagnosis in the ambulance chart would be made mandatory for every patient. Although we cannot be precise on the exact 
number of missed diagnoses, we can at least conclude that the exact diagnosis sepsis was not documented in a large proportion of the EMS charts that were handed over to the ED. Second, we can only rely on vital signs registered in the ambulance chart. It is therefore possible that vital signs were assessed more frequently than we retrieved in the charts. This will not be an issue in future research as the assessments of vital signs will be registered automatically in our ambulances in the near future. Third, in our studies, we were only able to retrieve treatments that were registered in the ambulance chart. However, the registration of started treatments was rather reliable, for this registration is used for feedback on skills maintenance. Fourth, Dutch ambulance personnel are highly qualified and because the $\mathrm{Ne}-$ therlands is a densely populated country, the ambulance can transport all urgent patients to an ED within 45 minutes and non-urgent patients within 60 minutes. This is different from the situation in other countries. In spite of this limitations, our findings concerning the recognition of sepsis are in accordance with American studies [41, 52].

In conclusion, ambulance personnel is highly alert at diagnosing acute myocardial infarction or trauma and uses standardized early treatment protocols that has reduced mortality substantially, while mortality in these patients is lower than in sepsis patients [53, 54]. We conclude that the ambulance can also be a key player in reducing mortality in sepsis patients. In theory, ambulance personnel can recognize almost $80 \%$ of the sepsis patients and almost half of all sepsis patients are transported by ambulance. In addition, early start of treatment with oxygen and fluids is found to be effective. Therefore, improving knowledge of sepsis and adjustment of the current ambulance protocol prescribing a more aggressive assessment and treatment in the ambulance could improve both morbidity and mortality. In addition to treatment with oxygen and fluids, antibiotic treatment may also be started in the ambulance. This could prove beneficial, if the results of early antibiotic treatment in the hospital can be extrapolated to the pre-hospital phase, which is never investigated before. Therefore, to investigate this issue, the first prospective randomized study was launched in the Netherlands, which assesses the impact of early antibiotic treatment in the ambulance on mortality [46].

\section{Risk stratification of sepsis patients in the Emergency Department}

Risk stratification of sepsis patients at the ED is important to identify patients with the highest mortality risk. In addition, risk stratification leads to a more standardized and in-

dividualized emergency care, for the emergency care could be adjusted to the severity of illness in the individual patient. Sepsis can be classified as sepsis, severe sepsis or septic shock, which are associated with an increasing in-hospital mortality of $16 \%$ in sepsis to $46 \%$ in septic shock $[6,10,55]$. However, there are several other factors influencing mortality which are not included in this classification, for example age, comorbidity, performance status or laboratory values. In the last decades, several scores have been developed that quantify the severity of illness in sepsis patients includes these factors. However, these scores have been developed and validated in different populations and countries [56,57]. Also, they have often been designed and validated in the same cohort, which may lead to an overestimation of their discriminatory value [58-60]. In addition, the scores have 
often not been calibrated $[58,61]$. Before implementation in daily practice, a severity of illness score must meet the following three conditions [56,57]. First, it must have a high discriminatory value, which means that the score is able to differentiate between patients with a high and low risk, for example high or low mortality risk. When a score has an AUC of $0.70-0.80$ the discriminatory value is considered good, an AUC of 0.80-0.90 is considered very good [62]. Second, the score must be well calibrated. Calibration is the degree of concordance between the prediction of a outcome and the observed outcome in the study population. Therefore, external validation of a new developed score is important to obviate this problem. Third, the score must be feasible, which means that the score is easy applicable in the whole population, without performing unnecessary and possibly harmful tests to complete the score.

In chapter 5, we investigated the discriminatory value of the MEDS, CURB-65, APACHE II, RAPS and REMS, for 28-day mortality in sepsis patients $(n=600)$. All five severity scores had very good to good discriminatory value, with AUCs ranging from 0.82 (MEDS) to 0.70 (RAPS). The discriminatory value of the MEDS was significantly higher than that of the RAPS and REMS, but not higher compared to the CURB-65 and APACHE II. The calibration of the MEDS and CURB-65 was rather well in the lowest risk levels, while the calibration was poor at higher estimated risks. In addition, the MEDS, CURB-65, RAPS and REMS were found to be highly feasible, because the items of the score were easily accessible at the ED. The ICU score APACHEII was not feasible at the ED with a complete score in $41.5 \%$ of the patients, and the discriminatory value and calibration of this score were not reliable, because the score was only complete in the sickest ED patients.

In conclusion, in chapter 5 the MEDS and CURB-65 are the most adequate risk stratification tools, but both need local recalibration, because of poor calibration in the higher risk category, to give accurate estimates of mortality risk at our ED, and probably at EDs of other hospitals in the Netherlands.

In the past decades, more severity of illness score than the five scores mentioned before have been developed. In our view, instead of developing new scores, the quality of the current scores should be expanded. As a first step, we validated fourteen severity scores in predicting 28 -day mortality in a large retrospective study ( $n=1230$, chapter 6$)$. In our study, we found four scores with good discriminatory value and feasibility, namely the abbMEDS, REMS, CURB-65 and NaURSE of which the abbMEDS performed best. In addition, the abbMEDS was well calibrated, in contrast to the REMS and CURB-65 that were less well calibrated in the lowest risk categories. Especially in the low risk group, it is important to be able to differentiate between low- and high-risk patients, because these patients might be safely treated as outpatients or with narrow-spectrum antibiotics. The NaURSE was well calibrated in the low risk category, but in the high risk category, the observed mortality was much lower than expected [63]. Further, although some of the scores include items regarding age, the predictive value of the scores have not been tested in elderly patients, who form an important part of the patients with sepsis. We found that the discriminatory value of the abbMEDS is maintained in elderly patients.

Ten scores were found to be less valuable at the ED, because of low discriminatory value and/or low feasibility, while two laboratory values (CRP/WBC) had no discriminatory value. 
The low predictive value of both laboratory tests is not surprising; it is not expected that the course of a complex disease, such as sepsis, can be predicted by a simple blood test.

In conclusion, the abbMEDS has the highest discriminatory value, also in elderly patients, in predicting 28-day mortality, is highly feasible and well calibrated.

To further expand the quality the risk stratification scores, prospective multicentre studies are necessary with consistent and well-defined populations and outcome measures. In addition, a sepsis-specific ED score would ideally be part of a clinical decision support system that not only discriminates well between those who will live and those who will die, but also guides antibiotic treatment (e.g. small or broad-spectrum) and disposition (i.e. whether a patient should be admitted to the hospital and if so, to what level of care). Eventually, such a clinical decision support system may lead to a higher level of emergency care in sepsis patients.

An example of a severity of illness score that discriminates well, but is also part of a clinical decision support system is the CURB-65. For patients with community-acquired pneumonia (CAP), the CURB-65 score stratifies patients into three risk categories (low, intermediate, high) that predict 28-day mortality well and guides antibiotic treatment and disposition [64]. The implementation of CURB-65 resulted in more standardized care and reduced the use of broad-spectrum antibiotics, while patient safety was maintained [65, 66]. It would be ideal, if the abbMEDS, the best score for patients with sepsis (chapter 6 ), could provide the same clinical guidance for patients with sepsis as the CURB-65 does for CAP.

In chapter 7, we performed a retrospective study with 725 sepsis patients that found that the abbMEDS categorized patients well with respect to two important clinical decisions, namely empirical antibiotic treatment and disposition. In addition, the abbMEDS performed better than the CURB-65. The most severely ill patients in the high risk category of the abbMEDS received almost exclusively IV broad-spectrum antibiotics. This is where the abbMEDS matched current clinical decisions (antibiotic treatment and disposition) better than the CURB-65. We further found that adequacy of empirical antibiotic treatment (identified micro-organisms are susceptible to empirical antibiotic treatment) was related to the abbMEDS risk categories. With increasing risk category of the abbMEDS, more patients were treated with adequate antibiotics. However, this was not the case for CURB-65, as $15.9 \%$ of the patients in the high risk category received inadequate treatment. The need for high adequacy is most urgent in this high risk category that includes the most severely ill patients [67]. For the less severely ill patients, a lower adequacy can be accepted, as confining microbial resistance to antibiotics is more important in this large group of patients.

In addition, the abbMEDS matched current management regarding disposition. With increasing risk category of the abbMEDS, more patients were admitted to the MCU/ICU, while the fraction of patients who were not admitted to the hospital decreased. Again, the CURB-65 did this less well.

There are some limitations in the chapters concerning risk stratification in sepsis patients at the ED. First, we depended on the documentation in the chart to retrieve all the necessary information, which can lead to misclassification. Some data (Glasgow Coma Scale, res- 
piratory rate) were deduced from other recorded information, and although this method is less accurate in this respect, other authors used similar solutions for these problems [58, 68]. Second, our studies were performed in sepsis patients who were assessed and treated by the internist, and therefore our findings may not be applicable to all sepsis patients at the ED. Third, like in most similar studies, the patients did not receive standardized treatment. Therefore, the observed differences in mortality rate between subgroups will not only depend on the severity of disease at the moment of presentation at the ED, but also on the treatment and the timing of treatment they received. Further, in chapter 7 , we considered this treatment and disposition by the physician as optimal. However, current clinical decisions might not be optimal as they are physician-dependent. However, our study reflect daily clinical practice and it is improbable that our management is less optimal than in other hospitals. Considering these limitations, our studies must been seen as exploratory ones. To our opinion, we showed that further research towards risk stratification and guidance of clinical decisions could improve both standardized care and mortality in sepsis patients at the ED.

In conclusion, the abbMEDS matches current clinical decisions in sepsis patients concerning empirical antibiotic treatment and disposition well, while this is less the case for the CURB-65.

We think that the abbMEDS has the potential to provide clinical decision support in patients presenting with sepsis and thereby increase the standard of care in sepsis patients. In order to develop the abbMEDS from a risk stratification to a decision support tool, future studies are needed to calibrate the abbMEDS in a prospective multicentre trial. Next, we have to confirm that the score can indeed appropriately and safely be used to provide this support on clinical decisions. Hopefully, the implementation of clinical decision support by abbMEDS will improve survival, reduce use of broad-spectrum antibiotics and reduce healthcare costs in sepsis patients. 


\section{References}

1. Dombrovskiy VY, Martin AA, Sunderram J, Paz HL. Rapid increase in hospitalization and mortality rates for severe sepsis in the United States: a trend analysis from 1993 to 2003. Crit Care Med. 2007;35(5):1244-50.

2. Angus DC, van der Poll T. Severe sepsis and septic shock. N Engl J Med. 2013;369(21):2063.

3. Seymour CW, Rea TD, Kahn JM, Walkey AJ, Yealy DM, Angus DC. Severe sepsis in pre-hospital emergency care: analysis of incidence, care, and outcome. Am J Respir Crit Care Med. 2012;186(12):126471.

4. Rivers EP, Katranji M, Jaehne KA, Brown S, Abou Dagher G, Cannon C, et al. Early interventions in severe sepsis and septic shock: a review of the evidence one decade later. Minerva Anestesiol. 2012;78(6):712-24.

5. Kumar A, Roberts D, Wood KE, Light B, Parrillo JE, Sharma S, et al. Duration of hypotension before initiation of effective antimicrobial therapy is the critical determinant of survival in human septic shock. Crit Care Med. 2006;34(6):1589-96.

6. Bone RC, Sibbald WJ, Sprung CL. The ACCP-SCCM consensus conference on sepsis and organ failure. Chest. 1992;101(6):1481-3.

7. Balk RA. Systemic inflammatory response syndrome (SIRS): where did it come from and is it still relevant today? Virulence. 2014;5(1):20-6.

8. Vincent JL, Abraham E, Annane D, Bernard G, Rivers E, Van den Berghe G. Reducing mortality in sepsis: new directions. Crit Care. 2002;6 Suppl 3:S1-18.

9. Klein Klouwenberg PM, Ong DS, Bonten MJ, Cremer OL. Classification of sepsis, severe sepsis and septic shock: the impact of minor variations in data capture and definition of SIRS criteria. Intensive Care Med. 2012;38(5):811-9.

10. Levy MM, Fink MP, Marshall JC, Abraham E, Angus D, Cook D, et al. 2001 SCCM/ESICM/ACCP/ATS/SIS International Sepsis Definitions Conference. Crit Care Med. 2003;31(4):1250-6.

11. Alberti C, Brun-Buisson C, Burchardi H, Martin C, Goodman S, Artigas A, et al. Epidemiology of sepsis and infection in ICU patients from an international multicentre cohort study. Intensive Care Med. 2002;28(2):108-21.

12. Brun-Buisson C, Meshaka P, Pinton P, Vallet B, Group ES. EPISEPSIS: a reappraisal of the epidemiology and outcome of severe sepsis in French intensive care units. Intensive Care Med. 2004;30(4):580-8.

13. Knaus WA, Sun X, Nystrom O, Wagner DP. Evaluation of definitions for sepsis. Chest. 1992;101(6):165662.

14. Jaimes F, Garces J, Cuervo J, Ramirez F, Ramirez J, Vargas A, et al. The systemic inflammatory response syndrome (SIRS) to identify infected patients in the emergency room. Intensive Care Med. 2003;29(8):1368-71.

15. Gille-Johnson P, Hansson KE, Gardlund B. Severe sepsis and systemic inflammatory response syndrome in emergency department patients with suspected severe infection. Scand J Infect Dis. 2013;45(3):186-93.

16. Vincent JL, Opal SM, Marshall JC, Tracey KJ. Sepsis definitions: time for change. Lancet. 2013;381(9868):774-5.

17. Kaukonen KM, Bailey M, Pilcher D, Cooper DJ, Bellomo R. Systemic inflammatory response syndrome criteria in defining severe sepsis. N Engl J Med. 2015;372(17):1629-38.

18. Sprung CL, Sakr Y, Vincent JL, Le Gall JR, Reinhart K, Ranieri VM, et al. An evaluation of systemic inflammatory response syndrome signs in the Sepsis Occurrence In Acutely III Patients (SOAP) study. Intensive Care Med. 2006;32(3):421-7.

19. Kaleta EJ, Clark AE, Cherkaoui A, Wysocki VH, Ingram EL, Schrenzel J, et al. Comparative analysis of PCR-electrospray ionization/mass spectrometry (MS) and MALDI-TOF/MS for the identification of bacteria and yeast from positive blood culture bottles. Clin Chem. 2011;57(7):1057-67.

20. Kaleta EJ, Clark AE, Johnson DR, Gamage DC, Wysocki VH, Cherkaoui A, et al. Use of PCR coupled with electrospray ionization mass spectrometry for rapid identification of bacterial and yeast bloodstream pathogens from blood culture bottles. J Clin Microbiol. 2011;49(1):345-53. 
21. KohImann R, Hoffmann A, Geis G, Gatermann S. MALDI-TOF mass spectrometry following short incubation on a solid medium is a valuable tool for rapid pathogen identification from positive blood cultures. Int J Med Microbiol. 2015;305(4-5):469-79.

22. Hoeboer SH, van der Geest PJ, Nieboer D, Groeneveld AB. The diagnostic accuracy of procalcitonin for bacteraemia: a systematic review and meta-analysis. Clin Microbiol Infect. 2015;21(5):474-81.

23. Hausfater P. Biomarkers and infection in the emergency unit. Med Mal Infect. 2014;44(4):139-45.

24. Clyne B, Olshaker JS. The C-reactive protein. J Emerg Med. 1999;17(6):1019-25.

25. Meisner M. Pathobiochemistry and clinical use of procalcitonin. Clin Chim Acta. 2002;323(1-2): 17-29.

26. Hatzistilianou M. Diagnostic and prognostic role of procalcitonin in infections. ScientificWorldJournal. 2010;10:1941-6.

27. Tsalik EL, Jaggers LB, Glickman SW, Langley RJ, van Velkinburgh JC, Park LP, et al. Discriminative value of inflammatory biomarkers for suspected sepsis. J Emerg Med. 2012;43(1):97-106.

28. Wacker C, Prkno A, Brunkhorst FM, Schlattmann P. Procalcitonin as a diagnostic marker for sepsis: a systematic review and meta-analysis. Lancet Infect Dis. 2013;13(5):426-35.

29. Pizzolato E, Ulla M, Galluzzo C, Lucchiari M, Manetta T, Lupia E, et al. Role of presepsin for the evaluation of sepsis in the emergency department. Clin Chem Lab Med. 2014;52(10):1395-400.

30. Romualdo LG, Torrella PE, Gonzalez MV, Sanchez RJ, Holgado AH, Freire AO, et al. Diagnostic accuracy of presepsin (soluble CD14 subtype) for prediction of bacteremia in patients with systemic inflammatory response syndrome in the Emergency Department. Clin Biochem. 2014;47(7-8):505-8.

31. Camussi G, Mariano F, Biancone L, De Martino A, Bussolati B, Montrucchio G, et al. Lipopolysaccharide binding protein and CD14 modulate the synthesis of platelet-activating factor by human monocytes and mesangial and endothelial cells stimulated with lipopolysaccharide. J Immunol. 1995;155(1):316-24.

32. Ulla M, Pizzolato E, Lucchiari M, Loiacono M, Soardo F, Forno D, et al. Diagnostic and prognostic value of presepsin in the management of sepsis in the emergency department: a multicenter prospective study. Crit Care. 2013;17(4):R168.

33. Liu B, Chen YX, Yin Q, Zhao YZ, Li CS. Diagnostic value and prognostic evaluation of Presepsin for sepsis in an emergency department. Crit Care. 2013;17(5):R244.

34. Chenevier-Gobeaux C, Trabattoni E, Roelens M, Borderie D, Claessens YE. Presepsin (sCD14-ST) in emergency department: the need for adapted threshold values? Clin Chim Acta. 2014;427:34-6.

35. Kibe S, Adams K, Barlow G. Diagnostic and prognostic biomarkers of sepsis in critical care. J Antimicrob Chemother. 2011;66 Suppl 2:ii33-40.

36. Clemmensen P, Wiberg S, Van't Hof A, Deliargyris EN, Coste P, Ten Berg J, et al. Acute stent thrombosis after primary percutaneous coronary intervention: insights from the EUROMAX trial (European Ambulance Acute Coronary Syndrome Angiography). JACC Cardiovasc Interv. 2015;8(1 Pt B):214-20.

37. Aoun RJ, Bendok BR, Zammar SG, Hamade YJ, Aguilar MI, Demaerschalk BM. From Delivering the Patient to the Hospital to Delivering the Hospital to the Patient: Acute Stroke Therapy in an Ambulance. World Neurosurg. 2015.

38. Assuncao M, Akamine N, Cardoso GS, Mello PV, Teles JM, Nunes AL, et al. Survey on physicians' knowledge of sepsis: do they recognize it promptly? J Crit Care. 2010;25(4):545-52.

39. Cronshaw HL, Daniels R, Bleetman A, Joynes E, Sheils M. Impact of the Surviving Sepsis Campaign on the recognition and management of severe sepsis in the emergency department: are we failing? Emerg Med J. 2011;28(8):670-5.

40. Suffoletto B, Frisch A, Prabhu A, Kristan J, Guyette FX, Callaway CW. Prediction of serious infection during prehospital emergency care. Prehosp Emerg Care. 2011;15(3):325-30.

41. Guerra WF, Mayfield TR, Meyers MS, Clouatre AE, Riccio JC. Early detection and treatment of patients with severe sepsis by prehospital personnel. J Emerg Med. 2013;44(6):1116-25.

42. Seymour CW, Band RA, Cooke CR, Mikkelsen ME, Hylton J, Rea TD, et al. Out-of-hospital characteristics and care of patients with severe sepsis: a cohort study. J Crit Care. 2010;25(4):553-62.

43. Studnek JR, Artho MR, Garner CL, Jr., Jones AE. The impact of emergency medical services on the ED care of severe sepsis. Am J Emerg Med. 2012;30(1):51-6.

44. Thijssen WA, Giesen PH, Wensing M. Emergency departments in The Netherlands. Emerg Med J. 2012;29(1):6-9. 
45. van der Wekken LC, Alam N, Holleman F, van Exter P, Kramer MH, Nanayakkara PW. Epidemiology of Sepsis and Its Recognition by Emergency Medical Services Personnel in the Netherlands. Prehosp Emerg Care. 2015.

46. ALAM N, Stassen, P.M., Nanayakkara, P. W. B. Optimaliseren ketenzorg bij patiënten met sepsis. NTVG. 2015;159:A8541.

47. Scott HF, Donoghue AJ, Gaieski DF, Marchese RF, Mistry RD. The utility of early lactate testing in undifferentiated pediatric systemic inflammatory response syndrome. Acad Emerg Med. 2012;19(11):127680 .

48. Seymour CW, Cooke CR, Mikkelsen ME, Hylton J, Rea TD, Goss CH, et al. Out-of-hospital fluid in severe sepsis: effect on early resuscitation in the emergency department. Prehosp Emerg Care. 2010;14(2):145-52.

49. Glickman SW, Cairns CB, Otero RM, Woods CW, Tsalik EL, Langley RJ, et al. Disease progression in hemodynamically stable patients presenting to the emergency department with sepsis. Acad Emerg Med. 2010;17(4):383-90.

50. Band RA, Gaieski DF, Hylton JH, Shofer FS, Goyal M, Meisel ZF. Arriving by emergency medical services improves time to treatment endpoints for patients with severe sepsis or septic shock. Acad Emerg Med. 2011;18(9):934-40.

51. Anonymous. [National protocol ambulance service, version 7.2] Landelijk protocol ambulancezorg versie $7.2 ; 2011$.

52. Seymour CW, Carlbom D, Engelberg RA, Larsen J, Bulger EM, Copass MK, et al. Understanding of sepsis among emergency medical services: a survey study. J Emerg Med. 2012;42(6):666-77.

53. Jollis JG, Roettig ML, Aluko AO, Anstrom KJ, Applegate RJ, Babb JD, et al. Implementation of a statewide system for coronary reperfusion for ST-segment elevation myocardial infarction. JAMA. 2007;298(20):2371-80.

54. Nathens AB, Jurkovich GJ, Rivara FP, Maier RV. Effectiveness of state trauma systems in reducing injury-related mortality: a national evaluation. J Trauma. 2000;48(1):25-30; discussion -1.

55. Rangel-Frausto MS, Pittet D, Costigan M, Hwang T, Davis CS, Wenzel RP. The natural history of the systemic inflammatory response syndrome (SIRS). A prospective study. JAMA. 1995;273(2):117-23.

56. Challen K, Goodacre SW. Predictive scoring in non-trauma emergency patients: a scoping review. Emerg Med J. 2011;28(10):827-37.

57. Calle P, Cerro L, Valencia J, Jaimes F. Usefulness of severity scores in patients with suspected infection in the emergency department: a systematic review. J Emerg Med. 2012;42(4):379-91.

58. Howell MD, Donnino MW, Talmor D, Clardy P, Ngo L, Shapiro NI. Performance of severity of illness scoring systems in emergency department patients with infection. Acad Emerg Med. 2007;14(8):709-14.

59. Olsson T, Lind L. Comparison of the rapid emergency medicine score and APACHE II in nonsurgical emergency department patients. Acad Emerg Med. 2003;10(10):1040-8.

60. Shapiro NI, Wolfe RE, Moore RB, Smith E, Burdick E, Bates DW. Mortality in Emergency Department Sepsis (MEDS) score: a prospectively derived and validated clinical prediction rule. Crit Care Med. 2003;31(3):670-5.

61. Chen YX, Li CS. Risk stratification and prognostic performance of the predisposition, infection, response, and organ dysfunction (PIRO) scoring system in septic patients in the emergency department: a cohort study. Crit Care. 2014;18(2):R74.

62. Šimundić A-M. Measures of diagnostic accuracy: basic definitions. http://www.ifcc.org/ifccfiles/ docs/190404200805.pdf.

63. Wilson AH, Kidd AC, Skinner J, Musonda P, Pai Y, Lunt CJ, et al. A simple 5-point scoring system, NaURSE ( $\mathrm{Na}+$, urea, respiratory rate and shock index in the elderly), predicts in-hospital mortality in oldest old. Age Ageing. 2014;43(3):352-7.

64. Lim WS, van der Eerden MM, Laing R, Boersma WG, Karalus N, Town Gl, et al. Defining community acquired pneumonia severity on presentation to hospital: an international derivation and validation study. Thorax. 2003;58(5):377-82.

65. Chalmers JD, Singanayagam A, Akram AR, Choudhury G, Mandal P, Hill AT. Safety and efficacy of CURB65-guided antibiotic therapy in community-acquired pneumonia. J Antimicrob Chemother. 2011;66(2):416-23. 
66. Singanayagam A, Chalmers JD. Severity assessment scores to guide empirical use of antibiotics in community acquired pneumonia. Lancet Respir Med. 2013;1(8):653-62.

67. Chen HC, Lin WL, Lin CC, Hsieh WH, Hsieh CH, Wu MH, et al. Outcome of inadequate empirical antibiotic therapy in emergency department patients with community-onset bloodstream infections. J Antimicrob Chemother. 2013;68(4):947-53.

68. Crowe CA, Kulstad EB, Mistry CD, Kulstad CE. Comparison of severity of illness scoring systems in the prediction of hospital mortality in severe sepsis and septic shock. J Emerg Trauma Shock;3(4):342-7. 

Summary 
Sepsis, which is defined as a systemic inflammatory response syndrome (SIRS) to an infection, is a broad clinical entity and a deadly disease. In order to intervene at an early stage, highly sensitive diagnostic criteria and/or tests are needed to recognize sepsis patients in time.

The sepsis definition had a lower diagnostic value than the diagnosis made by the physician at the ED in a general medical population at the ED, when the diagnosis at discharge was used as a reference. During stay at the ED and the hospital, many (19.3\%) patients crossed over from 'sepsis' to 'no sepsis' or vice versa. Therefore, good clinical judgment remains a prerequisite for diagnosing sepsis correctly. The current sepsis definition is still used in research because, there is no other extensively tested and superior definition of sepsis. Further studies are needed to optimize the diagnostic value of the sepsis definition at the Emergency Department.

Unfortunately, among all sorts of health care professionals, sepsis is a frequently missed diagnosis. In this thesis we found that sepsis is documented in the ambulance in about $60 \%$ of the patients with sepsis. The mortality of patients with non-documented sepsis in the ambulance was twice as high as in documented patients (25.7 vs. $12.9 \%$ ). However, almost $80 \%$ of all sepsis patients transported by the ambulance can be recognized when all vital signs are assessed by ambulance personnel and knowledge on sepsis is improved. Almost half of the sepsis patients who visit the internist at the ED have been transported by ambulance. These patients are older, more severely ill and have a higher mortality rate than patients transported otherwise. The severity of illness and the large number of sepsis patients transported by ambulance indicates that ambulance services may indeed play an important role in the improvement of care for sepsis patients.

Early identification of the severity of illness in sepsis patients reduces mortality. Therefore, risk stratification at the ED is important to guide both standardized and individual care in sepsis patients. In sepsis patients at the ED, of 14 severity of illness scores, the abbMEDS turned out to have the highest discriminatory value in predicting 28-day mortality, was highly feasible and well calibrated. This high discriminatory value of the abbMEDS was maintained in elderly patients. ICU scores turned out to be not feasible in the ED due to the high number of missing data. Traditional inflammation markers, C-reactive protein and white blood cell count, have no discriminatory value.

Another new finding is that the abbMEDS matches current clinical decisions in sepsis patients concerning antibiotic treatment (e. g. oral, intravenous small spectrum, intravenous broad spectrum) and disposition (i.e. whether a patient should be admitted to the hospital and to what type of ward) well. To improve the standard of care in sepsis patients, further studies regarding the ability of the abbMEDS to provide clinical decision support in decisions made by the physician at the ED are necessary. 
Samenvatting 
Sepsis is gedefinieerd als een systemisch inflammatoir respons syndroom (SIRS) op een infectie. Sepsis is een dodelijke ziekte en de ziekteverschijnselen zijn zeer variabel. Om vroege interventie mogelijk te maken is het noodzakelijk dat er zeer sensitieve diagnostische criteria en/of tests zijn om een patiënt met sepsis zo vroeg mogelijk te herkennen.

In een algemene interne populatie op de SEH had de definitie van sepsis een lagere diagnostische waarde dan de diagnose die gesteld werd door de arts op de Spoedeisende Hulp (SEH), wanneer de diagnose bij ontslag werd gebruikt als referentie. Tijdens verblijf op de SEH en in het ziekenhuis wisselden veel patiënten (19.3\%) tussen de diagnose 'sepsis' en 'geen sepsis' of vice versa. Daarom blijft de klinische blik van de arts een eerste vereiste om sepsis goed te kunnen diagnosticeren. De huidige sepsis definitie wordt nog steeds gebruikt in wetenschappelijk onderzoek, aangezien er nog geen andere uitgebreid geteste en superieure definitie van sepsis is. Vervolgstudies zijn nodig om de diagnostische waarde van de sepsis definitie op de SEH te optimaliseren.

Helaas wordt de diagnose sepsis regelmatig gemist door diverse gezondheidszorgmedewerkers. In dit proefschrift vonden wij dat de diagnose sepsis in de ambulancestatus in ongeveer $60 \%$ van de patiënten met sepsis wordt gedocumenteerd. De mortaliteit van patiënten waarbij sepsis niet was gedocumenteerd in de ambulancestatus was twee keer zo hoog dan in patiënten waar sepsis wel vervoerd was ( 25.7 vs. $12.9 \%$ ). Echter bijna $80 \%$ van alle sepsis patiënten die getransporteerd worden met de ambulance kunnen worden herkend wanneer alle vitale parameters gemeten worden door het ambulance personeel en de kennis van sepsis wordt verbeterd. Ongeveer de helft van de sepsis patiënten die zich presenteren op de SEH voor de interne geneeskunde worden vervoerd door de ambulance. Deze patiënten zijn ouder, zieker en hebben een hogere mortaliteit dan patiënten die met eigen vervoer naar de SEH komen. De ernst van de ziekte en het grote aantal sepsis patienten dat wordt vervoerd door de ambulance laat zien dat de ambulance een belangrijke rol kan spelen in de verbetering van zorg voor patiënten met sepsis.

Vroege identificatie van de ernst van de sepsis reduceert mortaliteit. Daarom is risicostratificatie op de SEH belangrijk om zowel gestandaardiseerde als individuele zorg te kunnen leveren aan patiënten met sepsis. Van een totaal van 14 scores heeft de abbMEDS het beste discriminerende vermogen om 28-dagen mortaliteit te voorspellen, de score was goed toepasbaar en goed gekalibreerd in patiënten met sepsis op de SEH. Dit discriminerende vermogen van de abbMEDS bleef ook aanwezig bij de oudere patiënt. Intensive care scores waren niet toepasbaar op de SEH vanwege een grote hoeveelheid missende data. Traditionele inflammatie markers zoals C-reactive protein en witte bloedcellen hebben geen discriminerend vermogen.

Een andere nieuwe bevinding is dat de abbMEDS goed overeenkomt met de huidige klinische beslissingen in zake patiënten met sepsis wat betreft antibiotica keuze (oraal, intraveneus small spectrum, intraveneus breed spectrum) en ziekenhuisopname (of de patiënt opgenomen wordt of naar huis gaat en indien patiënt opgenomen wordt naar welke afdeling hij gaat). Om de gestandaardiseerde zorg voor patiënten met sepsis te verbeteren zijn vervolgstudies nodig naar het vermogen van de abbMEDS om ondersteuning te geven in de klinische beslissingen die de arts op de SEH moet maken. 
Valorisation 
This thesis aimed to investigate the chain of emergency care in sepsis patients from the diagnosis sepsis to prehospital care and risk stratification at the Emergency Department (ED). This paragraph describes the consequences and innovations for daily clinical practice that (probably) arise from our findings.

\section{Relevance}

Sepsis, which is defined as a systemic inflammatory response syndrome (SIRS) to an infection, is a broad clinical entity and a deadly disease $[1,2]$. The incidence of sepsis has been estimated between 15 and 19 million per year worldwide, and overall mortality is about $30 \%[3,4]$. A quarter of the patients presenting at the ED for the internist in Maastricht University Medical Centre are diagnosed as having sepsis according (chapter 2). Sepsis is not only an important cause of death but puts significant strain on healthcare budgets as well [5]. In the last two decades, the implementation of the Surviving Sepsis Campaign (SSC), which aims to start treatment in sepsis patients early, has proved to reduce both mortality and sepsis-related costs $[6,7,8,9]$. This makes sense if you look at the sequence SIRS, sepsis, severe sepsis and septic shock as a process of falling dominoes. The mortality and morbidity caused by sepsis is time-dependent and increases with delay of adequate therapy [2].

\section{Consequences and innovations in current daily practice}

Only when sepsis is recognized, an early start of treatment according to the SSC is possible. The sepsis definition is an important tool to make a first differentiation between patients with and without sepsis. A clinical decision support system based on the sepsis definition could be helpful to detect sepsis earlier at the ED, and in the ambulance. However, the diagnostic accuracy of the current sepsis definition is not perfect. Therefore, in order to diagnose sepsis early, besides designing a decision support system, it is important to raise and maintain the awareness of sepsis in emergency physicians, because we showed that they perform better in diagnosing sepsis than the sepsis definition alone.

The guidelines for treatment provided by the SSC only address the treatment of the sepsis patients after arrival at the hospital and not during the pre-hospital phase [4]. However, the ambulance is the first encounter of the sepsis patient with the health care system. Since the outcome of sepsis is time-dependent, treatment of sepsis patients in the ambulance could reduce morbidity, mortality and thus, sepsis-related costs. In this thesis, we found that almost half of the sepsis patients who were assessed at the ED by the internist had been transported by the ambulance. These patients were older, more severely ill, were more often admitted to medium/intensive care units and had a higher mortality. This means that these patients would probably benefit most from early treatment of sepsis. The problem is that a substantial part of these patients sepsis are not documented to have sepsis. The mortality of these non-documented patients was twice as high than of the documented patients ( 25.7 vs. $12.9 \%$ ). Fortunately, the recognition of sepsis can be impro- 
ved, as we found that almost $50 \%$ of the missed patients could have been recognized if all vital signs had been assessed and knowledge of sepsis was improved.

Once recognized, early treatment in the ambulance with oxygen and intravenous fluids has indeed improved outcome [11, 12]. In addition to treatment with oxygen and fluids, antibiotic treatment may also be started in the ambulance. This could prove beneficial, if the results of early antibiotic treatment in the hospital can be extrapolated to the prehospital phase, which is never investigated before. Therefore, following the results of our studies, the first prospective randomized study, which assesses the impact of early antibiotic treatment in the ambulance on mortality and the number of (expensive) ICU admissions was recently launched in the Netherlands [13].

After arrival at the ED, the emergency physician has to identify the patients with the highest mortality risk. The mortality risk of the patients influences the resources, and thereby the costs, needed to treat the patient. The estimation of the mortality risk leads to standardized care in sepsis patients, but also to individualized and effective/efficient care (adjusted to the severity of disease and pre-existing morbidity and mortality risk). However, in the past decades, a large number of severity of sepsis scores have been developed and it is not clear which is best. We compared many scores and found that the abbMEDS is the best severity score in sepsis patients at the ED with regard to discriminatory value, feasibility and calibration. In addition, until now, the predictive value of the severity scores has not been established in the group of elderly patients, who form a major part of the patients with sepsis. Due to ageing of the population and their high concomitant comorbidity, there is an increase of elderly patients visiting the ED. We showed that the discriminatory value, feasibility and calibration of the abbMEDS is maintained in elderly patients.

Ideally, a sepsis-specific ED score would be part of a clinical decision support system that not only discriminates well between those who will live and those who will die, but also guides antibiotic treatment (e.g. small or broad-spectrum) and disposition (i.e. whether a patient should be admitted to the hospital and if so, to what level of care). Such guidance will lead to a more efficacious care, as was found in patients with community-acquired pneumonia (CAP). The CURB-65 score stratifies patients with CAP into three risk categories that predict 28-day mortality well and guide antibiotic treatment and disposition [14]. The implementation of CURB-65 resulted in more standardized care and reduced the use of broad-spectrum antibiotics, while patient safety was maintained $[15,16]$.

We found that the abbMEDS categorized patients with sepsis well with respect to two important clinical decisions, namely empirical antibiotic treatment and disposition. This is the first study towards the ability of the abbMEDS to guide clinical decisions. In order to develop the abbMEDS from a risk stratification to a decision support tool, future studies are needed to calibrate the score in a prospective multicentre trial. Next, we have to confirm that the score can indeed appropriately and safely be used to provide this support on clinical decisions. Hopefully, the implementation of clinical decision support by abbMEDS will improve survival, reduce use of broad-spectrum antibiotics and reduce healthcare costs in sepsis patients. 


\section{References}

1. Bone RC, Sibbald WJ, Sprung CL. The ACCP-SCCM consensus conference on sepsis and organ failure. Chest. 1992;101:1481-3.

2. Rangel-Frausto MS, Pittet D, Costigan M, Hwang T, Davis CS, Wenzel RP. The natural history of the systemic inflammatory response syndrome (SIRS). A prospective study. JAMA. 1995;273:117-23.

3. Adhikari NK, Fowler RA, Bhagwanjee S, Rubenfeld GD. Critical care and the global burden of critical illness in adults. Lancet. 2010;376:1339-46.

4. Angus DC, Linde-Zwirble WT, Lidicker J, Clermont G, Carcillo J, Pinsky MR. Epidemiology of severe sepsis in the United States: analysis of incidence, outcome, and associated costs of care. Crit Care Med. 2001;29:1303-10.

5. Bakker J, Levi M, van Hout BA, van Gestel A. Sepsis, a complicated syndrome with major medical and social consequences. Ned Tijdschr Geneeskd. 2004;148:975-8.

6. Rivers E, Nguyen B, Havstad S, Ressler J, Muzzin A, Knoblich B, et al. Early goal-directed therapy in the treatment of severe sepsis and septic shock. The New England journal of medicine. 2001;345(19):136877.

7. Puskarich MA, Marchick MR, Kline JA, Steuerwald MT, Jones AE. One year mortality of patients treated with an emergency department based early goal directed therapy protocol for severe sepsis and septic shock: a before and after study. Critical care. 2009;13(5):R167.

8. Levy MM, Dellinger RP, Townsend SR, Linde-Zwirble WT, Marshall JC, Bion J, et al. The Surviving Sepsis Campaign: results of an international guideline-based performance improvement program targeting severe sepsis. Critical care medicine. 2010;38(2):367-74.

9. Tromp M, Tjan DH, van Zanten AR, Gielen-Wijffels SE, Goekoop GJ, van den Boogaard M, et al. The effects of implementation of the Surviving Sepsis Campaign in the Netherlands. The Netherlands journal of medicine. 2011;69(6):292-8.

10. Dellinger RP, Carlet JM, Masur H, Gerlach H, Calandra T, Cohen J, et al. Surviving Sepsis Campaign guidelines for management of severe sepsis and septic shock. Crit Care Med. 2004 Mar;32(3):858-73.

11. Seymour CW, Cooke CR, Mikkelsen ME, Hylton J, Rea TD, Goss CH, et al. Out-of-hospital fluid in severe sepsis: effect on early resuscitation in the emergency department. Prehosp Emerg Care. 2010;14(2):145-52.

12. Band RA, Gaieski DF, Hylton JH, Shofer FS, Goyal M, Meisel ZF. Arriving by emergency medical services improves time to treatment endpoints for patients with severe sepsis or septic shock. Academic emergency medicine : official journal of the Society for Academic Emergency Medicine. 2011;18(9):934-40.

13. Alam N, Stassen, P.M., Nanayakkara, P. W. B. Optimaliseren ketenzorg bij patiënten met sepsis. NTVG. 2015;159:A8541.

14. Lim WS, van der Eerden MM, Laing R, Boersma WG, Karalus N, Town Gl, et al. Defining community acquired pneumonia severity on presentation to hospital: an international derivation and validation study. Thorax. 2003;58(5):377-82.

15. Chalmers JD, Singanayagam A, Akram AR, Choudhury G, Mandal P, Hill AT. Safety and efficacy of CURB65-guided antibiotic therapy in community-acquired pneumonia. The Journal of antimicrobial chemotherapy. 2011;66(2):416-23.

16. Singanayagam A, Chalmers JD. Severity assessment scores to guide empirical use of antibiotics in community acquired pneumonia. The lancet Respiratory medicine. 2013;1(8):653-62. 
Appendix 
Table 1 Converted values

\begin{tabular}{|c|c|}
\hline Category or text value & Converted value \\
\hline \multicolumn{2}{|l|}{ Respiratory rate } \\
\hline Normopnea, or $10-20$ & $14 /$ minute \\
\hline Tachypnea or $>20 /$ minute & 30/minute \\
\hline Abnormal & 25/minute \\
\hline \multicolumn{2}{|l|}{ Heart rate } \\
\hline Normal & 70/minute \\
\hline Tachycardia & $120 /$ minute \\
\hline \multicolumn{2}{|l|}{ Systolic blood pressure } \\
\hline Normal or $\geq 90$ & $120 \mathrm{mmHg}$ \\
\hline \multicolumn{2}{|l|}{ Glasgow Coma Scale } \\
\hline Normal & 15 \\
\hline \multicolumn{2}{|l|}{ Temperature } \\
\hline Normal & $37.0 \div \mathrm{C}$ \\
\hline Fever & $39.0 \div \mathrm{C}$ \\
\hline $\begin{array}{l}\text { Missing value in ambulance } \\
\text { chart and fever at ED }\end{array}$ & $39.0 \div \mathrm{C}$ \\
\hline
\end{tabular}

Table 2 Independent factors influencing mortality in all sepsis patients

\begin{tabular}{|c|c|c|c|c|}
\hline & \multicolumn{2}{|l|}{ Univariate } & \multicolumn{2}{|l|}{ Multivariate } \\
\hline & $\mathrm{HR}(95 \% \mathrm{Cl})$ & p-value & HR (95\% Cl) & p-value \\
\hline Age (per year) & $1.04(1.02-1.05)$ & $<0.0001$ & $1.03(1.01-1.04)$ & 0.001 \\
\hline Malignancy & $1.2(0.8-1.9)$ & 0.29 & & \\
\hline Cytotoxic medication & $1.0(0.6-1.8)$ & 0.87 & & \\
\hline $\begin{array}{l}\text { Immune suppressive } \\
\text { medication }\end{array}$ & $0.6(0.4-1.0)$ & 0.04 & $0.9(0.6-1.6)$ & 0.80 \\
\hline Means of transportation & $2.7(1.7-4.1)$ & $<0.0001$ & $1.4(0.9-2.4)$ & 0.15 \\
\hline \multicolumn{5}{|l|}{ Sepsis severity } \\
\hline Severe sepsis & $2.6(1.6-4.1)$ & $<0.0001$ & $1.6(1.0-2.6)$ & 0.08 \\
\hline Septic shock & $6.0(3.4-10.5)$ & $<0.0001$ & $3.1(1.6-6.0)$ & 0.001 \\
\hline Length of ED stay & $1.002(1.000-1.005)$ & 0.04 & $\begin{array}{r}1.003(1.000- \\
1.005)\end{array}$ & 0.03 \\
\hline
\end{tabular}


Table 3 Independent factors of influence on documentation of sepsis

\begin{tabular}{|l|l|l|l|l|}
\hline \multicolumn{2}{|l|}{ Univariate } & \multicolumn{1}{l|}{ Multivariate } \\
\hline & OR (Cl) & p-value & OR (Cl) & p-value \\
\hline Urgency of the journey* & $0.6(0.3-0.9)$ & 0.01 & $0.5(0.3-0.9)$ & 0.02 \\
\hline Assessments & $10.8(6.3-18.6)$ & $<0.0001$ & $11.2(5.2-24.4)$ & $<0.0001$ \\
\hline Body temperature & $0.3(0.1-0.5)$ & $<0.0001$ & $0.5(0.2-1.1)$ & 0.07 \\
\hline SIRS & $3.9(2.0-7.4)$ & $<0.0001$ & $0.7(0.3-1.8)$ & 0.43 \\
\hline None & & & \\
\hline All & & \\
\hline $\begin{array}{l}\text { OR = Odds ratio; Cl = confidence interval. } \\
* \text { Non-urgent (B) journey is reference. }\end{array}$
\end{tabular}

Table 4 Main reasons of missing values

\begin{tabular}{|c|c|c|c|c|c|}
\hline $\begin{array}{l}\text { Missing } \\
\text { items (\%) }\end{array}$ & $\begin{array}{l}\text { Arterial } \\
\text { blood } \\
\text { gas }\end{array}$ & Lactate & $\begin{array}{l}\text { Other } \\
\text { laboratory } \\
\text { values }\end{array}$ & Laboratory values of the scores & $\begin{array}{l}\text { Vital } \\
\text { para- } \\
\text { meters }\end{array}$ \\
\hline \multicolumn{6}{|c|}{ Sepsis-specific ED scores } \\
\hline MEDS & - & - & 20.0 & Leukocyte bands, platelet count & - \\
\hline $\begin{array}{l}\text { Abb- } \\
\text { MEDS }\end{array}$ & - & - & 1.1 & Platelet count & - \\
\hline PIRO & - & 79.6 & 24.5 & Bands, urea, platelet count & 0.7 \\
\hline mSSS & 57.9 & 79.6 & 34.0 & $\begin{array}{l}\text { WBC, platelet count, creatinine, } \\
\text { bilirubin, glucose, lactate }\end{array}$ & 1.6 \\
\hline \multicolumn{6}{|l|}{ ICU scores } \\
\hline mSAPS II & - & - & 75.9 & $\begin{array}{l}\text { WBC, sodium, potassium, urea, } \\
\text { bilirubin }\end{array}$ & 1.9 \\
\hline SOFA & 57.9 & - & 30.5 & $\begin{array}{l}\text { Platelet count, creatinine, } \\
\text { bilirubin }\end{array}$ & 0.7 \\
\hline APACHE & 57.9 & - & 15.6 & $\begin{array}{l}\text { WBC, hematocrit, creatinine, } \\
\text { sodium, potassium }\end{array}$ & 2.0 \\
\hline \multicolumn{6}{|c|}{ Non-disease-specific ED scores } \\
\hline REMS & - & - & - & & 3.9 \\
\hline $\begin{array}{l}\text { Shock } \\
\text { Index }\end{array}$ & - & - & - & & 0.1 \\
\hline \multicolumn{6}{|c|}{ Other scores } \\
\hline RAPS & - & - & - & & 0.8 \\
\hline CURB-65 & - & - & 4.7 & Urea & 0.7 \\
\hline PSI & 57.9 & - & 8.4 & $\begin{array}{l}\text { Hematocrit, sodium, urea, } \\
\text { glucose }\end{array}$ & 1.4 \\
\hline NaURSE & - & - & 3.3 & Urea & 0.1 \\
\hline MEWS & - & - & - & & 2.0 \\
\hline
\end{tabular}


Table 5 Calibration per risk category per score

\begin{tabular}{|c|c|c|}
\hline Total score & $\begin{array}{l}\text { Mortality (\%) } \\
\text { Original study }\end{array}$ & $\begin{array}{c}\text { Mortality (\%) } \\
\text { Our study }\end{array}$ \\
\hline \multicolumn{3}{|c|}{ AbbMEDS (14) } \\
\hline $0-4$ & 1.6 & 3.8 \\
\hline $5-12$ & 23.4 & 18.3 \\
\hline$>12$ & 59.0 & 51.3 \\
\hline \multicolumn{3}{|l|}{ REMS (6) } \\
\hline $0-1$ & 0 & 3.6 \\
\hline $2-3$ & 0 & 3.4 \\
\hline $4-5$ & 0 & 9.6 \\
\hline $6-7$ & 3.8 & 10.1 \\
\hline $8-9$ & 3.4 & 17.2 \\
\hline $10-11$ & 8.9 & 19.5 \\
\hline $12-13$ & 25.5 & 25.9 \\
\hline $14-15$ & 63.6 & 43.2 \\
\hline$>16$ & 78.6 & 77.8 \\
\hline \multicolumn{3}{|c|}{ CURB-65 (25) } \\
\hline $0-1$ & 1.5 & 5.6 \\
\hline 2 & 9.2 & 14.9 \\
\hline $3-5$ & 22.0 & 29.3 \\
\hline \multicolumn{3}{|c|}{ NaURSE (30) } \\
\hline 0 & 9.5 & 6.0 \\
\hline 1 & 19.9 & 12.3 \\
\hline 2 & 34.4 & 36.2 \\
\hline $3-4$ & 83.4 & 44.8 \\
\hline \multicolumn{3}{|c|}{$\begin{array}{l}\text { AbbMEDS = abbreviated mortality in emergency department sep- } \\
\text { sis; REMS = Rapid Emergency Medicine Score; CURB-65 = Confu- } \\
\text { sion, urea, respiratory rate, blood pressure and age }>65 \text {; NaURSE = } \\
\text { Sodium, urea, respiratory rate, Shock Index in the elderly. }\end{array}$} \\
\hline
\end{tabular}


Table 6 The abbMEDS (left panel) and CURB-65 score (right panel) with risk categories $(5,9)$

\begin{tabular}{|l|r|l|r|}
\hline abbMEDS item & Score & CURB-65 item & Score \\
\hline Terminal disease & 6 & Confusion & 1 \\
\hline Respiratory difficulty & 3 & Urea $>7$ mmol/l & 1 \\
\hline Septic shock & 3 & Respiratory rate $>30 / \mathrm{min}$ & 1 \\
\hline Thrombocytes $<150 \times 109$ cells/liter & 3 & $\begin{array}{l}\text { Blood pressure } \\
\text { (SBP }<90 \text { or DBP } \leq 60 \mathrm{mmHg})\end{array}$ & 1 \\
\hline Age $>65$ years & 3 & Age $\geq 65$ years & 1 \\
\hline Lower respiratory tract infection & 2 & & \\
\hline Nursing home resident & 2 & & \\
\hline Altered mental state & 2 & & \\
\hline Total & 24 & Total & 5 \\
\hline Risk category & Score & Risk category & Score \\
\hline low risk & $0-4$ & low risk & $0-1$ \\
\hline intermediate risk & $5-12$ & intermediate risk & 2 \\
\hline high risk & $13-24$ & high risk & $3-5$ \\
\hline
\end{tabular}

Table 7 Different groups of antibiotics

\begin{tabular}{|l|l|l|}
\hline Oral & IV narrow-spectrum & IV broad-spectrum \\
\hline Amoxicillin & Amoxicillin & Amoxicillin/clavulanate \\
\hline Amoxicillin/clavulanate & Ciprofloxacin & $\begin{array}{l}\text { Amoxicillin/clavulanate }+ \\
\text { gentamicin }\end{array}$ \\
\hline Ciprofloxacin & Cotrimoxazole & Cefalozin \\
\hline Cotrimoxazole & Flucloxacillin & Cefotaxime \\
\hline Doxycycline & Penicillin & Ceftazidime \\
\hline Flucloxacillin & Clindamycin & Ceftriaxone \\
\hline Nitrofurantoin & & Cefuroxime \\
\hline & & Ciprofloxacin + flucloxacillin \\
\hline $\begin{array}{l}\text { In case of allergy/contra } \\
\text { indication: }\end{array}$ & & Ciprofloxacin + gentamicin \\
\hline Azithromycin & & Clindamycin + ciprofloxacin \\
\hline Clarithromycin & & Levofloxacin \\
\hline Erythromycin & & Meropenem \\
\hline & & Moxifloxacin \\
\hline & & Piperacillin/tazobactam \\
\hline & & Vancomycin + ciprofloxacin \\
\hline
\end{tabular}


Table 8 Positive cultures per site and most commonly identified pathogens

\begin{tabular}{|l|r|l|}
\hline Site of culture & \multicolumn{1}{|l|}{$\mathbf{n}(\%)$} & Most common pathogen (\%) \\
\hline Urine & $155(48.9)$ & Escherichia coli (61.8) \\
\hline Blood & $98(30.9)$ & Escherichia coli (32.7) \\
\hline Wound/skin & $42(13.2)$ & Staphylococcus aureus (28.6) \\
\hline Sputum & $22(6.9)$ & Haemophilus influenzae (27.3) \\
\hline
\end{tabular}


Dankwoord 
Promoveren doe je nooit alleen en dit proefschrift is dan ook tot stand gekomen dankzij de samenwerking met velen. Sommigen wil ik hierbij in het bijzonder noemen.

Ten eerste wil ik uiteraard dr. P. M. Stassen bedanken. Beste Patricia, ontzettend bedankt voor de geweldige begeleiding de afgelopen jaren. Ik heb ontzettend veel van je geleerd, niet alleen op het gebied van research, maar ook in de kliniek. Jouw onophoudelijke enthousiasme en doorzettingsvermogen hebben mij over heel wat hobbels heen geholpen. De afgelopen jaren heb ik je soms misschien tot wanhoop gedreven, maar je bleef altijd open staan voor mijn vragen en stimuleerde mij tot het zoeken van oplossingen. Je correctiesnelheid van mijn artikelen was fenomenaal. Ik heb fijn met je samengewerkt zowel op professioneel als persoonlijk vlak. Het is voor mij een eer om je eerste promovendus te zijn. Bedankt voor alles!

Prof. dr. C. D. A. Stehouwer, beste Coen, hartelijk bedankt voor de begeleiding van deze promotie. Je hebt me de kans gegeven om meer inzicht te krijgen in epidemiologie en wetenschappelijk onderzoek. De les die je me hebt gegeven over het schrijven van een wetenschappelijk artikel komt nog elke keer op het juiste moment naar boven. Je beoordelingen van de artikelen waren snel, maar bovenal kritisch en volledig. De samenwerking op zowel professioneel als persoonlijk vlak heb ik als zeer prettig ervaren. Bedankt hiervoor.

De leden van de beoordelingscommissie, prof. dr. C. P. Van Schayck, prof. dr. A. E. Boonen, prof. dr. H. R. Haak, prof. dr. H. A. H. Kaasjager en dr. P. W. B. Nanayakkara wil ik bedanken voor het kritisch doorlezen van mijn proefschrift en de bereidheid mij te opponeren tijdens de verdediging van mijn proefschrift.

Mijn dank gaat ook uit naar alle WESP-studenten die betrokken zijn geweest bij het samenstellen van de databases en mee hebben gedacht, gediscussieerd en geschreven: Mariëlle Bakker, Jeanette Duijst, Farah Leijten, Maud Hermans, Michelle Hilderink, Maaike Groenewoudt, Niki Troost, Judith Stoffers, Joris Heyligen en Jan Tegtmeier. Bedankt voor al jullie inspanningen.

Yolande Keulemans, wil ik hartelijk bedanken voor haar hulp met het opzetten van het onderzoek, met name aan het begin van het hele proces. Hella Borggreve, hartelijk bedankt voor de begeleiding op de weg van de wetenschap, maar ook in de (poli)kliniek.

Alle leden van de vakgroep Interne Geneeskunde binnen het MUMC, maar in het bijzonder mijn directe begeleiders binnen de acute interne geneeskunde die ik nog niet genoemd heb wil ik bedanken voor de hulp en ondersteuning bij mijn onderzoek. Dirk, Floris, Richard, Ronald, Roger, hierbij wil ik jullie ook bedanken voor de begeleiding die jullie, ieder vanuit jullie eigen achtergrond, mij hebben gegeven in de kliniek. Ik zal altijd terugkijken op mijn opleiding in Maastricht als op een bijzonder mooie periode in mijn leven.

Dorien we hebben samen een leuke en leerzame tijd gehad als fellow, het was helaas zo voorbij. Het was fijn om jou als sparringpartner te hebben. Ondanks de afstand hoop ik dat we onze discussies kunnen blijven voortzetten. 
Beste Andrea, een arts kijkt heel anders naar een studie dan een epidemioloog. De discussies die we met elkaar hebben gehad waren zeer verhelderend. Dank voor je inzicht en hulp.

Beste Marloes, hierbij wil ik je bedanken voor je heldere inzicht en kritiek. Je reacties waren snel en volledig.

De collega's op de Spoedeisende Hulp wil ik hartelijk danken voor hun hulp bij het verzamelen van de data, in het bijzonder Guido Vandergraesen.

Ook hartelijk dank aan Jochen Jansen en zijn collega's bij de Regionale Ambulance Voorziening Zuid-Limburg (RAV-ZL), de ambulancestudies waren zonder hen niet van de grond gekomen.

Hierbij wil ik ook mijn huidige collega's in het VU Medisch Centrum bedanken dat zij mij de gelegenheid hebben gegeven om dit proefschrift te voltooien.

Opleiding en promotie is echter niet het enige in het leven, daarom wil ik ook mijn vrienden en familie bedanken. Ik kan ze niet allemaal met name noemen, maar een paar uitzonderingen zal ik toch maken.

De leden van de Christengemeente Bunde-Meerssen hebben mijn jaren in Maastricht tot een onvergetelijke tijd gemaakt. Zij stonden altijd voor mij klaar en daarvoor wil ik ze hartelijk bedanken.

Beste Arisja, je bent al jaren mijn beste vriendin. We hebben de afgelopen jaren samen veel gelachen en soms gehuild. Je hebt me altijd gesteund in de keuzes die ik heb gemaakt. Het spijt me dat ik weer vertrokken ben uit Maastricht, ik hoop dat we in de toekomst weer wat dichter bij elkaar wonen. Bedankt voor alles!

Mijn ouders (mama in herinnering) wil ik bedanken voor de onophoudelijke steun en belangstelling tijdens mijn opleiding en promotie. Jullie stonden altijd in alles voor mij klaar. Beste Chris, grote broer, hartelijk dank dat je jouw grafisch talent hebt losgelaten op dit proefschrift.

Beste Florus, broertje, ik vind het nog steeds leuk dat je ook in de gezondheidszorg bent terechtgekomen.

Lieve Jacco, halverwege mijn promotie kwam jij in mijn leven. Hiermee ben ik nog steeds gelukkig. Hartelijk bedankt voor alle begrip, steun en zondagse wandelingen, ondanks dat je vaak wel moeite moest doen om me op de been te krijgen na een week van werken en onderzoek. Hopelijk wordt dit voor jou ook makkelijker nu het af is.

Bovenal dank aan God die mij de gezondheid, kracht en wijsheid heeft gegeven om zowel de opleiding als deze promotie te voltooien. 



\section{Curriculum Vitae}


Asselina Aaltje Roest werd geboren op 18 april 1983 te Huizen. Na het behalen van haar VWO diploma aan het Erfgooiers College te Huizen in 2001 begon ze aan haar studie Geneeskunde aan de Universiteit van Amsterdam waar zij in 2007 haar artsexamen behaalde. Van november 2007 tot april 2009 is zij werkzaam geweest als arts-assistent Interne Geneeskunde niet in opleiding in het Rode Kruis Ziekenhuis te Beverwijk. Van april 2009 tot april 2010 is zij werkzaam geweest als arts-assistent niet in opleiding op de Intensive Care van het VU Medisch Centrum te Amsterdam. Daarna is zij in mei 2010 begonnen met de opleiding Interne Geneeskunde in het Academisch Ziekenhuis Maastricht met als opleider Prof. Dr. C. D. A. Stehouwer en plaatsvervangend opleider Prof. Dr. R. P. Koopmans. In 2013 startte zij met haar aandachtsgebied Acute Interne Geneeskunde en haar onderzoek naar Emergency Care in Sepsis Patients onder leiding van prof. dr. C. D. A. Stehouwer en dr. P. M. Stassen, waarvan de resultaten hebben geleid tot dit proefschrift. Sinds mei 2015 is zij werkzaam als Internist met als aandachtsgebied Acute Interne Geneeskunde in het VU Medisch Centrum te Amsterdam. 

